\title{
A Topos for Algebraic Quantum Theory
}

\author{
Chris Heunen $^{1}$, Nicolaas P. Landsman ${ }^{1}$, Bas Spitters ${ }^{2, \star}$ \\ ${ }^{1}$ Radboud Universiteit Nijmegen, Institute for Mathematics, Astrophysics, \\ and Particle Physics, Toernooiveld 1, 6525 ED Nijmegen, The Netherlands. \\ E-mail: landsman@math.ru.nl \\ 2 Eindhoven University of Technology, Department of Mathematics and Computer Science, \\ P.O. Box 513, 5600 MB Eindhoven, The Netherlands
}

Received: 7 April 2008 / Accepted: 17 April 2009

Published online: 21 July 2009 - (c) The Author(s) 2009. This article is published with open access at Springerlink.com

\section{Dedicated to Ieke Moerdijk, at his 50th birthday}

\begin{abstract}
The aim of this paper is to relate algebraic quantum mechanics to topos theory, so as to construct new foundations for quantum logic and quantum spaces. Motivated by Bohr's idea that the empirical content of quantum physics is accessible only through classical physics, we show how a noncommutative $\mathrm{C}^{*}$-algebra of observables $A$ induces a topos $\mathcal{T}(A)$ in which the amalgamation of all of its commutative subalgebras comprises a single commutative $\mathrm{C}^{*}$-algebra $\underline{A}$. According to the constructive Gelfand duality theorem of Banaschewski and Mulvey, the latter has an internal spectrum $\underline{\Sigma}(\underline{A})$ in $\mathcal{T}(A)$, which in our approach plays the role of the quantum phase space of the system. Thus we associate a locale (which is the topos-theoretical notion of a space and which intrinsically carries the intuitionistic logical structure of a Heyting algebra) to a $\mathrm{C}^{*}$-algebra (which is the noncommutative notion of a space). In this setting, states on $A$ become probability measures (more precisely, valuations) on $\underline{\Sigma}$, and self-adjoint elements of $A$ define continuous functions (more precisely, locale maps) from $\underline{\Sigma}$ to Scott's interval domain. Noting that open subsets of $\underline{\Sigma}(\underline{A})$ correspond to propositions about the system, the pairing map that assigns a (generalized) truth value to a state and a proposition assumes an extremely simple categorical form. Formulated in this way, the quantum theory defined by $A$ is essentially turned into a classical theory, internal to the topos $\mathcal{T}(A)$.
\end{abstract}

These results were inspired by the topos-theoretic approach to quantum physics proposed by Butterfield and Isham, as recently generalized by Döring and Isham.

\footnotetext{
^ Supported by N. W. O.
} 
Motto: 'Ces “nuages probabilistes", remplaçant les rassurantes particules matérielles d'antan, me rappellent étrangement les élusifs "voisinages ouverts" qui peuplent les topos, tels des fantômes évanescents, pour entourer des "points" imaginaires.' (A. Grothendieck [43]) ${ }^{1}$

\section{Introduction}

This introduction is intended for both mathematical physicists and topos theorists. We apologize in advance for stating the obvious for one or the other of these groups at various points, but we hope that most of it is interesting to both communities.

1.1. The logic of classical physics. In classical mechanics, the logical structure of a physical system is encoded in its phase space $M$. Indeed, elementary propositions (also called 'yes-no questions' in physics) correspond to suitable subsets of $M$ (such as all sets, all measurable sets or all regular open sets), and the logical connectives are given by the standard set-theoretic operations. This makes the logic of the system 'spatial'; its realization as a Boolean algebra (i.e. a distributive lattice with 0 and 1 in which every element has a complement) confirms the 'classical' nature of the situation [76].

Physicists do not usually describe a system in the above way. Instead, they work with observables $a: M \rightarrow \mathbb{R}$, like position or energy. (Such functions might be arbitrary, or else required to be measurable or continuous as appropriate.) From that perspective, the elementary propositions are of the form $a \in \Delta$, where $\Delta \subseteq \mathbb{R}$ is a (arbitrary, measurable, or regular open) subset of the reals.

Either way, a state of the system may be construed as a catalogue of answers to all yes-no questions about the systems. We concentrate on pure states $\rho \in M$, which provide sharp (as opposed to probabilistic) answers. In the first description, a proposition $U \subseteq M$ is true (equivalently, the answer to the corresponding question is 'yes') iff $\rho \in \bar{U}$. In the second description, $a \in \Delta$ is true for a state $\rho$ iff $a(\rho) \in \Delta$, i.e. iff $\rho \in a^{-1}(\Delta)$. Thus propositions of the second type fall into equivalence classes $[a \in \Delta]=a^{-1}(\Delta)$. As these are subsets of $M$, this leads us back to the purely spatial picture of the first description.

This truth assignment has a very simple categorical description. We regard $M$ as an object in the category Sets of all sets as objects and all functions as arrows, and interpret $\rho$ as an arrow (in fact, a monomorphism) $1 \stackrel{\rho}{\longrightarrow} M$, where 1 is any singleton. A subset $U \subseteq M$ may alternatively be described by its characteristic function $\chi_{U}: M \rightarrow\{0,1\}$; anticipating the convention in topos theory, we relabel $\{0,1\}$ as $\Omega$ and regard this as an object in Sets. Composition of $1 \stackrel{\rho}{\longrightarrow} M$ and $M \stackrel{x_{a^{-1}(\Delta)}}{\longrightarrow} \Omega$ then yields an arrow

$$
1 \stackrel{\langle\rho, a \in \Delta\rangle}{\longrightarrow} \Omega \quad=\quad 1 \stackrel{\rho}{\longrightarrow} M \stackrel{\chi_{a}-1(\Delta)}{\longrightarrow} \Omega,
$$

i.e. we have defined

$$
\langle\rho, a \in \Delta\rangle=\chi_{a^{-1}(\Delta)} \circ \rho
$$

1 "These "probability clouds", replacing the reassuring material particles of before, remind me strangely of the elusive "open neighborhoods" that populate the topoi, like evanescent phantoms, to surround the imaginary "points".' 
The image of 1 under this map is a point of $\Omega$, which is precisely the above truth value of the proposition $a \in \Delta$ in the state $\rho$ (provided we identify $\{0,1\}$ with \{false, true\}).

It is important for what follows to reformulate this description in terms of the topology $\mathcal{O}(M)$ of $M$ (i.e. its collection of opens) instead of $M$ itself. This makes sense if the subsets $U \subseteq M$ above are open, which in our second description is the case if the observables $a$ are continuous and the value sets $\Delta \subseteq \mathbb{R}$ are open as well. Hence $a^{-1}(\Delta)$ is an arrow $1 \frac{a^{-1}(\Delta)}{\longrightarrow} \mathcal{O}(M)$ in Sets, but $\rho \in M$ is now represented by the "state subobject' $[\rho] \subseteq \mathcal{O}(M)$ given by

$$
[\rho]=\{V \in \mathcal{O}(M) \mid \rho \in V\}=\left\{V \in \mathcal{O}(M) \mid \delta_{\rho}(V)=1\right\},
$$

where $\delta_{\rho}$ is the Dirac measure on $M$ concentrated at $\rho$. We describe this object by its characteristic function $\chi_{[\rho]}: \mathcal{O}(M) \rightarrow \Omega$. The pairing map then becomes

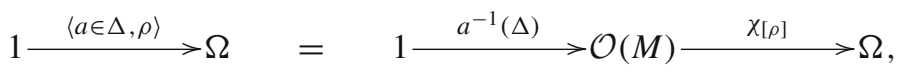

or, in other words,

$$
\langle a \in \Delta, \rho\rangle=\chi_{[\rho]} \circ a^{-1}(\Delta) .
$$

The reader may verify that $\langle a \in \Delta, \rho\rangle=\langle\rho, a \in \Delta\rangle$, so that our second categorical description of the state-proposition pairing is equivalent to the first. More generally, if $\mu$ is a probability measure on $M$, we might define a state object $[\mu]$ by replacing the Dirac measure $\delta_{\rho}$ in (3) by $\mu$, i.e.

$$
[\mu]=\{V \in \mathcal{O}(M) \mid \mu(V)=1\} .
$$

In physics, $\mu$ plays the role of a mixed state (unless it is a point measure, in which case it happens to be pure). Like the pure state $\rho$ (or rather its associated probability measure $\left.\delta_{\rho}\right)$, the mixed state $\mu$ defines a characteristic function $\chi_{[\mu]}: \mathcal{O}(M) \rightarrow \Omega$. The latter, however, turns out not to share the attractive logical properties of $\chi_{[\rho]} \equiv \chi_{\delta_{\rho}}$ (unless $\mu$ is pure); see Subsect. 1.5.

1.2. Spatial quantum logic. The goal of this paper is to generalize this situation to quantum mechanics. In particular, we wish to find a spatial notion of quantum logic. This objective will be accomplished by:

1. Identifying an appropriate notion of a quantum phase 'space' $\Sigma$.

2. Defining suitable 'subsets' of $\Sigma$ that act as elementary logical propositions of quantum mechanics.

3. Describing observables and states in terms of $\Sigma$.

4. Associating a proposition $a \in \Delta$ (and hence a 'subset' $[a \in \Delta]$ of $\Sigma$ ) to an observable $a$ and an open subset $\Delta \subseteq \mathbb{R}$.

5. Finding a pairing map between pure states and 'subsets' of $\Sigma$ (and hence between states and propositions of the type $a \in \Delta$ ).

In the last step, a state assigns a particular truth value to a given proposition; this is supposed to give empirical content to the formalism. The codomain $\Omega$ of the pairing map in item 5, which may be called the 'truth object' of the theory, is by no means obvious and identifying it is explicitly part of the question. Certainly, 20th century physics shows that the 'classical' choice $\Omega=\{0,1\}$ is out of the question.

The formulation of these objectives and the associated program goes back to von Neumann, who also famously proposed the following extremely elegant solution: 
1. A quantum phase space is a Hilbert space $H$.

2. Elementary propositions correspond to closed linear subspaces of $H$.

3. Observables are selfadjoint operators on $H$ and pure states are unit vectors in $H$.

4. The closed linear subspace $[a \in \Delta]$ is the image $E(\Delta) H$ of the spectral projection $E(\Delta)$ defined by $a$ and $\Delta$ (provided the latter is measurable).

5. The pairing map takes values in $[0,1]$ and is given by the "Born rule" $\langle\Psi, a \in \Delta\rangle=$ $(\Psi, E(\Delta) \Psi)$.

Thus subsets of phase space became closed linear subspaces of Hilbert space, which, as Birkhoff and von Neumann [10] noticed, form a lattice $\mathcal{L}(H)$ under inclusion as partial order. However, this lattice fails to be Boolean, basically because it is nondistributive. Nonetheless, Birkhoff and von Neumann interpreted the lattice operations $\wedge$ and $\vee$ as 'and' and 'or', as in the classical case, and argued that the departure from the Boolean structure (and hence from classical logic) meant that one had to deal with a new kind of logic, which they aptly called quantum logic. This looked highly innovative, but on the other hand it conservatively preserved the spatial nature of the logic of classical physics, in that the logical structure of propositions is still associated with the spatial (i.e. Hilbert space) structure of the theory.

Attractive and revolutionary as this spatial quantum 'logic' may appear [54,55,70, 76], it faces severe problems. The main logical drawbacks are:

- Due to its lack of distributivity, quantum 'logic' is difficult to interpret as a logical structure.

- In particular, despite various proposals no satisfactory implication operator has been found (so that there is no deductive system in quantum logic).

- Quantum 'logic' is a propositional language; no satisfactory generalization to predicate logic has been found.

Quantum logic is also problematic from a physical perspective. Since (by various theorems [14] and wide agreement) quantum probabilities do not admit an ignorance interpretation, $[0,1]$-valued truth values attributed to propositions by pure states via the Born rule cannot be regarded as sharp (i.e. $\{0,1\}$-valued) truth values muddled by human ignorance. This implies that, if $x=[a \in \Delta]$ represents a quantum-mechanical proposition, it is wrong to say that either $x$ or its negation holds, but we just do not know which of these alternatives applies. However, in quantum logic one has the law of the excluded middle in the form $x \vee x^{\perp}=1$ for all $x$. Thus the formalism of quantum logic does not match the probabilistic structure of quantum theory responsible for its empirical content.

In fact, the above argument suggests that it is intuitionistic logic rather than quantum logic that is relevant in quantum mechanics ( $c f$. [4]). More generally, as argued in particular by Butterfield and Isham $[17,20]$, the fact that pure states fail to define truth assignments in the usual binary sense (i.e. true or false) renders the entire notion of truth in quantum mechanics obscure and calls for a complete reanalysis thereof [34-38]. As also probably first recognized by the same authors, such an analysis can fruitfully be attempted using topos theory, whose internal logic is indeed intuitionistic.

From our perspective, another reason why topos theory offers itself on a silver tray in our search for a spatial quantum logic lies in the interplay between spatial and logical structures inherent in topos theory, as exemplified by the opening words of the renowned textbook by Mac Lane and Moerdijk:

A startling aspect of topos theory is that it unifies two seemingly wholly distinct mathematical subjects: on the one hand, topology and algebraic geometry and on the other hand, logic and set theory. 
We refer to $[42,50,51,63]$ for accounts of topos theory; see also $[9,57,65]$ for historical details. Briefly, a topos is a category in which one can essentially reason as in the category Sets of all sets (with functions as arrows), except for the fact that the logic is intuitionistic and the axiom of choice is generally not available. Briefly, the mathematics underlying topos theory is constructive.

Specifically, a topos is a category with the following ingredients:

1. Terminal object. This is an object called 1 (unique up to isomorphism) such that for each object $A$ there is a unique arrow $A \rightarrow 1$, generalizing the singleton set in the category Sets.

2. Pullbacks. These generalize the fibered product $B \times{ }_{A} C=\{(b, c) \in B \times C \mid f(b)=$ $g(c)\}$ of $B \stackrel{f}{\longrightarrow} A$ and $C \stackrel{g}{\longrightarrow} A$ in Sets into a pullback square with appropriate universality property. Cartesian products are a special case.

3. Exponentials. These generalize the idea that the class $B^{A}$ of functions from a set $A$ to a set $B$ is itself a set, and hence an object in Sets, equipped with the evaluation map ev : $A \times B^{A} \rightarrow B$.

4. Subobject classifier. This generalizes the idea that one may characterize a subset $A \subseteq B$ by its characteristic function $\chi_{A}: B \rightarrow\{0,1\}$. Subsets generalize to subobjects, i.e. monic ("injective") arrows $A \longmapsto B$, and in a topos there exists an object $\Omega$ (the subobject classifier) with associated arrow $1 \stackrel{\top}{\longrightarrow} \Omega$ ("truth") such that for any subobject $A \longmapsto B$ there is a unique arrow $B \stackrel{\chi_{A}}{\longrightarrow} \Omega$ for which $B \stackrel{f}{\longleftarrow} A \longrightarrow 1$ is a pullback of $B \stackrel{\chi_{A}}{\longrightarrow} \Omega$ and $1 \stackrel{\top}{\longrightarrow} \Omega$. Conversely, given any arrow $B \stackrel{\chi}{\longrightarrow} \Omega$ there exists a subobject $A \longmapsto B$ of $B$ (unique up to isomorphism) whose classifying arrow $\chi_{B}$ equals $\chi$. The subobject classifier in a topos plays the role of a "multi-valued truth object", generalizing the simple situation in Sets, where $\Omega=\{0,1\}=\{$ false, true $\}$; see (2) and subsequent text.

We assume that our topoi are cocomplete and have a natural numbers object.

1.3. Generalized notions of space. Our first objective in the list at the beginning of Subsect. 1.2, i.e. the identification of an appropriate notion of a quantum phase 'space', will be met by a combination of two profound notions of generalized space that have been around for some time.

1. First, let us recall the strategy of noncommutative geometry [25,26]. One starts with the replacement of a compact topological space $X$ by the associated algebra of complex-valued continuous functions $C(X, \mathbb{C})$. If $X$ fails to be Hausdorff, this step loses information, but if it is, one may recover $X$ from the commutative $\mathrm{C}^{*}$-algebra $C(X, \mathbb{C})$ as its Gelfand spectrum. This yields a duality between the category of compact Hausdorff spaces and the category of unital commutative $\mathrm{C}^{*}$-algebras: nothing is lost, but nothing is gained either by abstracting spaces as commutative $\mathrm{C}^{*}$-algebras. The thrust of noncommutative geometry, then, is to allow $\mathrm{C}^{*}$-algebras to be noncommutative without losing the spatial perspective. That this can be done is impressive enough, but as the logical situation is obscured by moving from commutative to noncommutative $\mathrm{C}^{*}$-algebras, further ideas are needed (at least if one is interested in quantum logic).

2. A second approach to generalizing topological spaces would be to replace $X$ by its topology $\mathcal{O}(X)$. This has a natural lattice structure under inclusion, and in fact 
defines a highly structured kind of lattice known as a frame. This is a complete distributive lattice such that $x \wedge \bigvee_{\lambda} y_{\lambda}=\bigvee_{\lambda} x \wedge y_{\lambda}$ for arbitrary families $\left\{y_{\lambda}\right\}$ (and not just for finite ones, in which case the said property follows from the definition of a distributive lattice). For example, if $X$ is a topological space, then the topology $\mathcal{O}(X)$ of $X$ is a frame with $U \leqslant V$ if $U \subseteq V$. A frame homomorphism preserves finite meets and arbitrary joins; this leads to the category Frm of frames and frame homomorphisms.

Abstracting frames $\mathcal{O}(X)$ coming from a topological space to general frames is a genuine generalization of the concept of a space, as plenty of frames exist that are not of the form $\mathcal{O}(X)$. A simple example is the frame $\mathcal{O}_{\text {reg }}(\mathbb{R})$ of regular open subsets of $\mathbb{R}$, i.e. of open subsets $U$ with the property $\neg \neg U=U$, where $\neg U$ is the interior of the complement of $U$. This may be contrasted with the situation for unital commutative $\mathrm{C}^{*}$-algebras, which, as just recalled, are all of the form $C(X)$. Moreover, far from obscuring the logical structure of space, the generalization of spaces by frames rather explains and deepens this structure.

Indeed, a frame is a complete Heyting algebra, with its intrinsic structure of an intuitionistic propositional logic. Here a Heyting algebra is a distributive lattice $\mathcal{L}$ with a map $\rightarrow: \mathcal{L} \times \mathcal{L} \rightarrow \mathcal{L}$ satisfying $x \leqslant(y \rightarrow z)$ iff $x \wedge y \leqslant z$, called implication $[42,63,80]$. Every Boolean algebra is a Heyting algebra, but not vice versa; in fact, a Heyting algebra is Boolean iff $\neg \neg x=x$ for all $x$, which is the case iff $\neg x \vee x=\top$ for all $x$. Here negation is a derived notion, defined by $\neg x=(x \rightarrow \perp)$. For example, $\mathcal{O}_{\text {reg }}(\mathbb{R})$ is Boolean, but $\mathcal{O}(\mathbb{R})$ is not. In general, the elements of a Heyting algebra form an intuitionistic propositional logic under the usual logical interpretation of the lattice operations.

A Heyting algebra is complete when arbitrary joins (i.e. sups) and meets (i.e. infs) exist. A complete Heyting algebra is essentially the same thing as a frame, for in a frame one may define $y \rightarrow z=\bigvee\{x \mid x \wedge y \leqslant z\}$. Conversely, the infinite distributivity law in a frame is automatically satisfied in a Heyting algebra. The set of subobjects of a given object in a topos forms a complete Heyting algebra (as long as the topos in question is defined "internal to Sets"), generalizing the fact that the set of subsets of a given set is a Boolean algebra. The subobject classifier of such a topos is a complete Heyting algebra as well; in fact, these two statements are equivalent. (Note, however, frame maps do not necessarily preserve the implication $\rightarrow$ defining the Heyting algebra structure, as can already be seen in examples of the type $f^{-1}: \mathcal{O}(Y) \rightarrow \mathcal{O}(X)$, where $f: X \rightarrow Y$ is continuous [63]. Consequently, negation may not be preserved by frame maps either.)

The category Loc of locales is the opposite category to Frm, i.e. it has the same objects but all arrows go in the opposite direction. Some topos theorists write $X$ for a locale and $\mathcal{O} X$ or $\mathcal{O}(X)$ for the same object seen as a frame [48,63,80]. Apart from the already unfortunate fact that this notation is applied also when $\mathcal{O}(X)$ does not stand for the opens of a space $X$ but denotes a general frame, it fails to distinguish between a topological space $X$ and the associated locale (i.e. the frame $\mathcal{O}(X)$ seen as a locale). Nonetheless, this notation often leads to elegant expressions and we will heavily use it.

If $X$ and $Y$ are spaces, a continuous map $f: X \rightarrow Y$ induces a frame map $f^{-1}$ : $\mathcal{O}(Y) \rightarrow \mathcal{O}(X)$ and hence an arrow $\mathcal{O}(X) \rightarrow \mathcal{O}(Y)$ in Loc, simply defined as $f^{-1}$ read in the opposite direction. We write the latter arrow in Loc simply as $f: X \rightarrow Y$. In general, an arrow in Frm is written as $f^{-1}: \mathcal{O}(Y) \rightarrow \mathcal{O}(X)$ (whether or not the frames in question come from topological spaces and if so, whether or not $f^{-1}$ is indeed the pullback of a continuous function between these spaces), and the corresponding arrow in Loc is denoted by $f: X \rightarrow Y$. Similarly, we will write $C(X, Y)$ for 
$\operatorname{Hom}_{\text {Loc }}(X, Y)=\operatorname{Hom}_{\mathrm{Frm}}(\mathcal{O}(Y), \mathcal{O}(X))$. In particular, for a locale $X, C(X, \mathbb{C})$ will denote the set of frame maps $\mathcal{O}(\mathbb{C}) \rightarrow \mathcal{O}(X)$.

1.4. Points and opens of locales. An element of a set $X$ (and hence $a$ fortiori also a point of a topological space $X$ ) may be identified with an arrow $* \rightarrow X$, where $*$ is a given singleton (for simplicity we write $*$ instead of the more usual $\{*\}$ ). The same goes for locales $X$, so that by definition a point of a locale $X$ is a locale map $p: * \rightarrow X$, hence a frame map $p^{-1}: \mathcal{O}(X) \rightarrow \mathcal{O}(*) \cong\{0,1\}=\Omega$; recall that the subobject classifier in Sets, seen as a topos, is $\Omega=\{0,1\}$ and note that $*$ is precisely the locale associated to $\Omega$, as our notation $\Omega=\mathcal{O}(*)$ has indicated.

A point of a locale $X$ being defined as a locale map $* \rightarrow X$ or as the corresponding frame map $\mathcal{O}(X) \rightarrow \Omega$, an open of $X$ is defined as a locale map $X \rightarrow S$, where $S$ is the locale defined by the so-called Sierpinski space, i.e. $\{0,1\}$ with $\{1\}$ as the only open point. The corresponding frame map $\mathcal{O}(S) \rightarrow \mathcal{O}(X)$ is determined by its value at 1 (since $\emptyset \mapsto \emptyset$ and $\{0,1\} \mapsto X$ ), so that we may simply look at opens in $X$ as arrows $1 \rightarrow \mathcal{O}(X)$ (where the singleton 1 is seen as the terminal object in Sets). Clearly, if $X$ is a genuine topological space with associated frame $\mathcal{O}(X)$ of opens, then each such map $1 \rightarrow \mathcal{O}(X)$ corresponds to an open subset of $X$ in the usual sense. Using this concept, the set $\operatorname{Pt}(X)$ of points of a locale $X$ may be topologized in a natural way, by declaring its opens to be the sets of the form

$$
\operatorname{Pt}(U)=\left\{p \in \operatorname{Pt}(X) \mid p^{-1}(U)=1\right\},
$$

where $U \in \mathcal{O}(X)$ is some open. We say that a locale $X$ is spatial if it is isomorphic (in the category of locales) to $\operatorname{Pt}(X)$ (more precisely, to the locale associated to the frame $\mathcal{O}(\operatorname{Pt}(X))$ in the above topology). Conversely, a topological space $X$ is called sober if it is homeomorphic to $\operatorname{Pt}(X)$ (which, with the notation introduced above, really stands for the space of points of the locale associated to the frame $\mathcal{O}(X))$. It is useful to know that $X$ is sober when it is Hausdorff. If $X$ is sober, any frame map $\varphi: \mathcal{O}(Y) \rightarrow \mathcal{O}(X)$ is induced by a continuous map $f: X \rightarrow Y$ as $\varphi=f^{-1}$. This provides additional justification for the notation $f^{-1}: \mathcal{O}(Y) \rightarrow \mathcal{O}(X)$ for a general frame map, and $f: X \rightarrow Y$ for the associated locale map. See [63, Sect. IX.3] for a very clear exposition of all this.

For example, referring to Subsect. 1.1, the characteristic function $\chi_{[\rho]}: \mathcal{O}(M) \rightarrow \Omega$ introduced below (3) is easily checked to define a frame map. Renaming this map as $\chi_{[\rho]} \equiv \rho^{-1}$, the associated locale map $\rho: * \rightarrow M$ is therefore a point of the locale $M$ in the above sense. In this special case, such a point may also be described by an arrow $1 \rightarrow M$, where 1 is the terminal object in Sets and $M$ denotes $M$ as a set rather than as a locale. This notion of points as elements of sets will be avoided in what follows.

Thus frames and locales are two sides of the same coin: the elements $1 \rightarrow \mathcal{O}(X)$ of the Heyting algebra $\mathcal{O}(X)$ are the opens of the associated locale $X$, to be thought of as propositions, whereas the points of the locale correspond to models of the logical theory defined by these propositions. See [51,63] and especially [80] for a very clear explanation of this perspective. More precisely, recall that geometric propositional logic stands for the following fragment of intuitionistic propositional logic $[51,63,80]$. A formula $\varphi$ in propositional geometric logic must be built from atomic propositions using the symbols $\top$ (for "truth"), $\wedge$ (for "and"), and $\vee$ (for "or"), where $\vee$ but not $\wedge$ is allowed to carry an infinite index set. (This may be motivated by the remark that to verify a proposition $\vee_{\lambda \in \Lambda} p_{\lambda}$, one only needs to find a single $p_{\lambda}$, whereas to verify $\wedge_{\lambda \in \Lambda} p_{\lambda}$ the truth of each $p_{\lambda}$ needs to be established, an impossible task in practice when $\Lambda$ is infinite.) Sequents or axioms must take the form $\varphi \rightarrow \psi$, where $\varphi$ and $\psi$ are formulae. 
A frame $\mathcal{O}(X)$, then, defines a geometric propositional theory whose propositions correspond to opens in $X$, combined by logical connectives given by the lattice operations in $\mathcal{O}(X)$ à la Boole. This quite literally holds in the case of classical physics discussed in Subsect. 1.1, where the opens of the locale $M$ are just the opens $U$ of $M$ as a topological space in the naive sense, construed as propositions "the system is in a state located within $U$ ". Conversely, a propositional geometric theory $\mathbb{T}$ has an associated Lindenbaum algebra $\mathcal{O}([\mathbb{T}])$, defined as the poset of formulae of $\mathbb{T}$ modulo provable equivalence, ordered by entailment. This poset turns out to be a frame, and the (standard) models of $\mathbb{T}$ (that by definition assign one of the two standard truth values 0 or 1 to the propositions of $\mathbb{T}$ in a consistent way) bijectively correspond to frame maps $\mathcal{O}([\mathbb{T}]) \rightarrow\{0,1\}$. Identifying $\{0,1\}$ with $\Omega=\mathcal{O}(*)$ as explained above, we see that a model of the theory $\mathbb{T}$ is the same thing as a point $* \rightarrow[\mathbb{T}]$ of the locale $[\mathbb{T}]$. More generally, one may consider a model of $\mathbb{T}$ in a frame $\mathcal{O}(Y)$ (generalizing the standard models where $Y=*$ ) to be a locale map $Y \rightarrow[\mathbb{T}]$.

\subsection{Locales in topoi. The generalization from topological spaces to frames is an impor-} tant step towards our goal, but it is not enough. Seeking further generality pertinent to quantum theory, one may proceed in at least two different ways. First, one may generalize locales to quantales [67]. This step leads to recognizable logical structures, but it does not relate well to the Copenhagen Interpretation of quantum mechanics we favour.

Instead, we pass from frames as special objects in the category of sets (as defined above) to frames in more general topoi. This is indeed possible, as all of the above concepts can be defined in any topos by using its internal language [63]; see [12] for details. In particular, in a topos $\mathcal{T}$ one may consider the category $\mathbf{F r m}_{\mathcal{T}}$ of internal frames and its opposite category $\operatorname{Loc}_{\mathcal{T}}$ of internal locales. The terminal object of the latter is the locale $*$ whose associated frame $\mathcal{O}(*)$ is the subobject classifier $\Omega$ of $\mathcal{T}$. Opens, points and models are then defined in exactly the same way as in Sets, as long as one realizes that the identification of $\Omega$ with $\{0,1\}$ and of $*$ with the singleton is peculiar to Sets.

In particular, a point of a locale $X$ in $\mathcal{T}$ is a frame map $\mathcal{O}(X) \rightarrow \Omega$, whereas an open in $X$ may be defined as an arrow $1 \rightarrow \mathcal{O}(X)$. The collection $\operatorname{Pt}(X)$ of a locale is still defined as the subobject of $\Omega^{\mathcal{O}(X)}$ corresponding to frame maps, its opens being given by interpreting (7) in the internal language of $\mathcal{T}$, where $U \in \mathcal{O}(X)$ is interpreted as an arrow $1 \stackrel{U}{\rightarrow} \mathcal{O}(X)$ and $p^{-1}(U)=1$ means that $p^{-1} \circ U=\top$, i.e. the truth arrow $\top: 1 \rightarrow \Omega$ in $\mathcal{T}$.

In any case, it is reassuring that topos theorists simply refer to 'internal' locales as 'spaces' $[52,53,66]$ : returning to the opening words from Mac Lane and Moerdijk quoted earlier, one might say that the unification in question is exemplified by the idea of an internal locale with its associated Heyting algebra structure.

Our quantum phase spaces $\underline{\Sigma}$, then, will be examples of locales in topoi. Their opens $1 \rightarrow \mathcal{O}(\underline{\Sigma})$ will correspond to the elementary propositions or yes-no questions about the system, and each physical state on the system will define a map $\mathcal{O}(\underline{\Sigma}) \rightarrow \underline{\Omega}$, where $\underline{\Omega}$ is the subobject classifier in the particular topos in which $\underline{\Sigma}$ is defined. It is important to note that such maps generally fail to be frame maps, i.e. they do not define models in the above sense. This phenomenon already arises in classical physics if one considers mixed rather than pure states; indeed, the map $\chi_{[\mu]}: \mathcal{O}(M) \rightarrow \Omega$ introduced below 6 fails to be a frame map (except when $\mu$ happens to be pure).

However, a fundamental difference between classical and quantum physics in this respect lies in the Kochen-Specker Theorem, which in its topos-theoretic incarnation 
(given in different versions in [20] and in Theorem 6 below) states that (generically) the quantum phase space $\Sigma$ has no points at all, although the quantum system has pure states (see Subsect. 4.1). Hence whereas pure states in classical physics - as defined in the usual sense through convexity - are also 'pure' in the logical sense, this is no longer the case in quantum physics.

Nonetheless, pairing states and propositions into an internal truth value, i.e. taking the subobject classifier to be the codomain of the pairing map, is a central goal of this work, which we share with (and adopted from) the work of Isham et al. [20,34]. Unlike real-number valued pairings (which from a logical perspective might be preferable), an $\Omega$-valued pairing avoids both the problems with the ignorance interpretation of the Born probabilities (see Subsect. 1.2) and the bizarre ontology of the so-called Many-Worlds interpretation of quantum mechanics $(c f$. $[14,17])$. A philosophical defence of this goal may also be found in [18]. However, the final verdict about its validity, or rather its relevance to physics, can only be given once the Born rule has been derived from our $\underline{\Omega}$-valued pairing, along with an appropriate interpretation of the Born probabilities. This derivation will be given in future work, in which the results of Sect. 6 of this paper will be combined with those in [61].

1.6. Basic construction. The two notions of generalized space just described, i.e. noncommutative $\mathrm{C}^{*}$-algebra $\mathrm{s}$ and locales in arbitrary topoi, will be related by one of the main constructions in this paper, which we summarize in this subsection. This construction associates a certain internal locale to a noncommutative $\mathrm{C}^{*}$-algebra (assumed unital), and hinges on three ideas:

1. Algebraic quantum theory $[39,44,58]$;

2. Constructive Gelfand duality [5-7,27,29];

3. Bohr's doctrine of classical concepts $[11,60,73]$.

From the first, we just adopt the methodology of describing a quantum system by a noncommutative $\mathrm{C}^{*}$-algebra $A$ (defined in the usual topos Sets). This move generalizes the usual Hilbert space framework of quantum theory and has the advantage of being able to incorporate superselection rules in infinite systems, as well as other limiting situations like the transition from quantum to classical mechanics (and back).

As to the second, it turns out that the notion of a $\mathrm{C}^{*}$-algebra makes sense in an arbitrary topos, so that one may, in particular, internalize commutative $\mathrm{C}^{*}$-algebras. Examples of such internal commutative $\mathrm{C}^{*}$-algebras arise from compact completely regular locales $([7,48]$, see also footnotes 2 and 3 below): if $X$ is such a locale in some topos $\mathcal{T}$, and if $\mathbb{C}$ is the locale defined by the complex numbers object in $\mathcal{T}$ (as in [7]), then the object $C(X, \mathbb{C})$ of all locale maps from $X$ to $\mathbb{C}$ is a commutative $\mathrm{C}^{*}$-algebra in $\mathcal{T}$ under natural operations. The Gelfand duality theorem of Banaschewski and Mulvey [7] states that, like in the case of the topos Sets, up to isomorphism these are the only examples of unital commutative $\mathrm{C}^{*}$-algebras: if $A$ is a unital commutative $\mathrm{C}^{*}$-algebra in a topos $\mathcal{T}$, there exists a compact completely regular locale $\Sigma$ such that $A \cong C(\Sigma, \mathbb{C})$. Here $\cong$ denotes isomorphism in the category $\mathcal{T}$ and the arrows implementing this isomorphism are $\mathrm{C}^{*}$-algebra maps. Moreover, this isomorphism extends to a categorical duality between compact completely regular locales and unital commutative $\mathrm{C}^{*}$-algebras in $\mathcal{T}$. We call the locale $\Sigma$ or $\Sigma(A)$ the Gelfand spectrum of $A$. It is defined up to isomorphism of locales.

Third, Niels Bohr's "doctrine of classical concepts" states that we can only look at the quantum world through classical glasses, measurement merely providing a "classical 
snapshot of reality". The combination of all such snapshots should then provide a complete picture. In Bohr's own words ([11], p. 209):

However far the phenomena transcend the scope of classical physical explanation, the account of all evidence must be expressed in classical terms. (...) The argument is simply that by the word experiment we refer to a situation where we can tell others what we have done and what we have learned and that, therefore, the account of the experimental arrangements and of the results of the observations must be expressed in unambiguous language with suitable application of the terminology of classical physics.

This doctrine has a transparent formulation in algebraic quantum theory, to the effect that the empirical content of a quantum theory described by a certain noncommutative $C^{*}$-algebra $A$ is contained in suitable commutative $C^{*}$-algebras associated to $A$. In the simplest case, which we study in this paper, these are simply the (unital) commutative $C^{*}$-subalgebras of $A$. (To understand classical behaviour in general, the pertinent commutative $C^{*}$-algebras have to be extracted from $A$ using limiting procedures like $\hbar \rightarrow 0$ or $N \rightarrow \infty$ [60].)

The following construction weaves these three threads together. Let $A$ be a unital $\mathrm{C}^{*}$-algebra (in the usual sense, i.e. in Sets) and let $\mathcal{C}(A)$ be the collection of its unital commutative $C^{*}$-subalgebras, partially ordered by inclusion. We regard the poset $\mathcal{C}(A)$ as a category, whose objects are the unital $C^{*}$-subalgebras $C \subseteq A$, and whose Hom-sets $\operatorname{Hom}_{\mathcal{C}(A)}(C, D)$ consist of a single arrow if $C \subseteq D$ and are empty otherwise. The category $\mathcal{C}(A)$ is a catalogue of all 'classical snapshots of reality' one may take of the quantum system described by $A$.

Recall that for any category $\mathcal{C}$, the topos Sets $^{\mathcal{C}}$ has functors $\mathcal{C} \rightarrow$ Sets as objects and natural transformations as arrows [63]. Put

$$
\mathcal{T}(A)=\operatorname{Sets}^{\mathcal{C}(A)} \text {. }
$$

The philosophical idea is that as observers we are confined to the topos $\mathcal{T}(A)$, whereas the physical system itself divinely exists in the ambient topos Sets. According to Bohr and Heisenberg, the system might seem to behave probabilistically from our limited classical perspective, but this behaviour is just a consequence of our confinement to $\mathcal{T}(A)$ ( $c f$. Theorem 14 below).

We will underline entities internal to $\mathcal{T}(A)$. It turns out that the tautological functor $\underline{A}: C \mapsto C$, which (with some abuse of notation) maps a unital commutative $C^{*}$-subalgebra $C$ of $A$ (seen as an object of the category $\mathcal{C}(A)$ ) into itself (seen as a set), is a unital commutative $\mathrm{C}^{*}$-algebra in $\mathcal{T}(A)$. We call $\underline{A}$ the Bohrification of $A$. It has an associated Gelfand spectrum $\underline{\Sigma}(\underline{A})$, which is a locale in $\mathcal{T}(A)$. The map $A \mapsto \underline{\Sigma}(\underline{A})$ associates a 'space' $\underline{\Sigma}(\underline{A})$ in the sense of topos theory to a 'space' $A$ in the sense of noncommutative geometry.

In principle, this construction leads to the solution of all five problems listed at the beginning of Subsect. 1.2:

1. The quantum phase space of the system described by $A$ is the locale $\underline{\Sigma} \equiv \underline{\Sigma}(\underline{A})$ in the topos $\mathcal{T}(A)$.

2. The "subsets" of the locale $\underline{\Sigma}$ acting as elementary propositions about $A$ are simply the 'opens' in $\underline{\Sigma}$, defined as arrows $1 \rightarrow \mathcal{O}(\underline{\Sigma})$ in $\mathcal{T}(A)$. Thus the quantum logic of $A$ is given by the Heyting algebra underlying $\underline{\Sigma}(\underline{A})$.

3. Observables $a \in A$ define locale maps $\delta(a): \underline{\Sigma} \rightarrow \underline{\mathbb{R}}$, where $\underline{\mathbb{R}}$ is the so-called interval domain. States $\rho$ on $A$ yield probability measures (more precisely, valuations) $\mu_{\rho}$ on $\underline{\Sigma}$. 
4. An open interval $\Delta \subseteq \mathbb{R}$ defines an arrow $\underline{1} \stackrel{\Delta}{\rightarrow} \mathcal{O}(\underline{\mathbb{R}})$ of $\mathcal{T}(A)$ (where $\underline{1}$ is the terminal object in $\mathcal{T}(A)$ ), which, composed with the map $\mathcal{O}(\underline{\mathbb{R}}) \stackrel{\underline{\delta}(a)^{-1}}{\longrightarrow} \mathcal{O}(\underline{\Sigma})$ underlying $\underline{\delta}(a)$, yields the desired proposition

$$
\underline{1} \stackrel{[a \in \Delta]}{\longrightarrow} \mathcal{O}(\underline{\Sigma}) \quad=\quad \underline{1} \stackrel{\Delta}{\longrightarrow} \mathcal{O}(\underline{\mathbb{R}}) \stackrel{\underline{\delta}(a)^{-1}}{\longrightarrow} \mathcal{O}(\underline{\Sigma}) .
$$

5. State-proposition pairing is defined exactly as in (4), i.e. by

$$
\underline{1} \stackrel{\langle a \in \Delta, \rho\rangle}{\longrightarrow} \underline{\Omega} \quad=\quad \underline{1} \stackrel{[a \in \Delta]}{\longrightarrow} \mathcal{O}(\underline{\Sigma}) \stackrel{\chi_{[\rho]}}{\longrightarrow} \underline{\Omega},
$$

where $\Omega$ is the subobject classifier of $\mathcal{T}(A)$ and $\chi_{[\rho]}$ is the characteristic map of the subobject $[\rho]$ of $\mathcal{O}(\underline{\Sigma})$ consisting of all opens $U$ of $\underline{\Sigma}$ with $\mu_{\rho}(U)=1$ (defined through the internal language of $\mathcal{T}(A)$ ).

The construction of $\underline{\delta}(a)$ is inspired by, and partly generalizes, the Daseinisation map of Döring and Isham ([35,36], cf. also Appendix B).

The subobject classifier $\underline{\Omega}$ is the functor $\mathcal{C}(A) \rightarrow$ Sets given by

$$
\underline{\Omega}(C)=\{S \subseteq \uparrow C \mid S \text { is an upper set }\},
$$

where for any poset $P$ an upper set in $P$ is a subset $U \subseteq P$ for which $x \in U$ and $x \leqslant y$ implies $y \in U$, and one writes $\uparrow x=\{y \in P \mid x \leqslant y\}$ for the so-called principal upper set on $x$. Note that $\underline{\Omega}(C)$ is a poset (and even a frame) under inclusion as partial ordering, with $\emptyset$ as bottom element, and $\uparrow C$ as top element. (One might think of the principal upper set $\uparrow C$ on the "classical snapshot of reality" $C$ as the collection of all finer versions of the knowledge present in $C$.) The subobject classifier $\underline{\Omega}$ is a (covariant) functor by stipulating that if $C \subseteq D$, then the induced map $\underline{\Omega}(C) \rightarrow \underline{\Omega}(D)$ is given by $S \mapsto S \cap \uparrow D$.

In this setup, we have taken Sets as the ambient topos. There are several reasons, however, one might want to consider other ambient topoi. Leaving the matter to future investigation, let us briefly indicate an important application. An algebraic quantum field theory (AQFT) [44] may be defined as a functor $(\mathcal{O}(M), \subseteq) \rightarrow$ CStar satisfying certain separability constraints, where $M$ is Minkowski space-time and $\mathcal{O}(M)$ is its set of opens [13]. Analogous to Theorem 5 below, an AQFT may then be shown to be given by a single $\mathrm{C}^{*}$-algebra in the presheaf topos $\operatorname{Sets}^{\mathcal{O}(M)}$ (though not a commutative one).

1.7. Internal and external language. We have repeatedly used the word 'internal' for a construction intrinsic to a certain topos $\mathcal{T}$; for example, $\underline{A}$ and its Gelfand spectrum $\underline{\Sigma}$ are internal to $\mathcal{T}(A)$, as is the Heyting algebra structure of $\underline{\Sigma}$. At this point, confusion may arise, for on the one hand the propositional logic carried by $\underline{\Sigma}$ is intuitionistic, while on the other hand all constructions (ranging from the initial $\mathrm{C}^{*}$-algebra $A$ to the locale $\underline{\Sigma}(\underline{A})$ as an object in the associated functor topos Sets $\left.^{\mathcal{C}(A)}\right)$ eventually arise from the topos Sets, whose underlying logic is classical.

To clarify this, we remark that it is a very important aspect of topos theory that one may, indeed, usually adopt two points of view: an external one and an internal one. External constructions are carried out using classical mathematics, which (at least for the topoi used in this paper) takes place in the familiar topos Sets (even if the constructions in question are concerned with some other topos). Internal constructions, on the other 


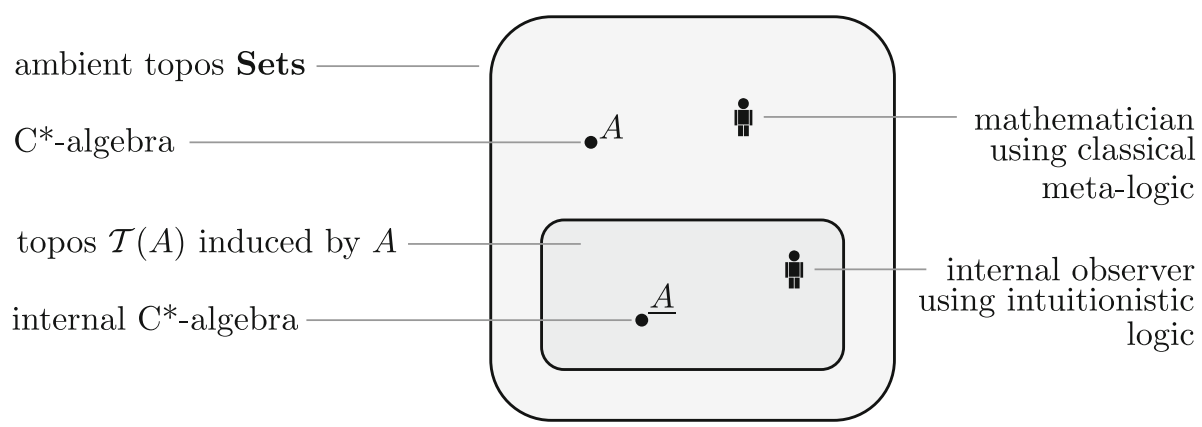

Fig. 1. Illustration of universes of discourse

hand, only use concepts intrinsic to the topos one is studying. This idea is formalized by the internal or Mitchell-Bénabou language associated with each topos [12,51,63]. This is a logical language that for many instances and purposes allows one to reason within a given topos as if it were the topos Sets. For example, one may employ the usual logical and set-theoretic symbols (the latter even if an object $X$ has no or few elements in the sense of arrows $1 \rightarrow X$ ), whose meaning is determined by the so-called Kripke-Joyal semantics associated with the Mitchell-Bénabou language. We will actually use this semantics in our theory of state-proposition pairing. However, using the internal language one may (in general) neither appeal to the law of excluded middle $x \vee \neg x=\top$, nor to the Axiom of Choice (although restricted versions thereof are sometimes valid). These limitations are a consequence of the fact that the internal language of a topos happens to be based on intuitionistic predicate logic (see $[12,51]$ for the precise rules).

Thus a topos can be seen as a universe of discourse, to which a mathematician or observer may wish to confine himself. On the other hand, even the internal language and associated logic can alternatively be studied externally with classical meta-logic. The various entities at play in our application of topos theory to quantum physics are illustrated in Fig. 1. This illustrates, in particular, that our quantum logic is meant to be the logic of an 'internal' observer, with all the restrictions this brings with it (whereas the quantum 'logic' of Birkhoff and von Neumann, to the extent it is a logic at all, rather pertains to a fictitious entity like Laplace's demon, to whose intellect 'nothing would be uncertain and the future just like the past would be present before its eyes.')

Let us give three closely related examples of internal versus external descriptions, each relevant to our logical approach to quantum theory.

First, a fundamental fact of topos theory is that the subobjects $\operatorname{Sub}_{\mathcal{T}}(A)$ of a given object $A$ in a topos $\mathcal{T}$ (with subobject classifier $\Omega_{\mathcal{T}}$ ) form a (complete) Heyting algebra.

- Externally, one simply looks at $\operatorname{Sub}_{\mathcal{T}}(A)$ as a set, equipped with the structure of a Heyting algebra in the category Sets.

- Internally, $\operatorname{Sub}_{\mathcal{T}}(A)$ is described as the exponential $\Omega_{\mathcal{T}}^{A}$ (or power 'set' $\mathcal{P}(A)$ ), which is a Heyting algebra object in $\mathcal{T}$. See [63, p. 201].

Second, as these Heyting algebras are complete, they are frames. The explicit internal description of a frame or locale is rather complicated as far as the completeness property of the underlying lattice is concerned [12]. However, if the topos $\mathcal{T}=\operatorname{Sh}(X)$ is that of sheaves on a locale $X$ (which, we recall, consists of those functors $F$ in $\operatorname{Sets}^{\mathcal{O}(X)^{\text {op }}}$ that satisfy a gluing condition stating that $F(U)$ can be computed from the $F\left(U_{i}\right)$ under any open covering $U=\cup_{i} U_{i}$ [63, Ch. II]), a simple external description is available $[49,53]$ 
(also $c f$. [51, Sect. C1.6]): a locale $\overline{\mathcal{L}}$ in $\operatorname{Sh}(X)$ is externally described by a locale map $f: \mathcal{L} \rightarrow X$ in Sets, with

$$
\mathcal{L}=\overline{\mathcal{L}}(X) .
$$

Furthermore, if $\overline{\mathcal{L}}_{1}$ and $\overline{\mathcal{L}}_{2}$ are locales in $\operatorname{Sh}(X)$ with external descriptions $f_{i}: \mathcal{L}_{i} \rightarrow X$, then an internal locale map $\bar{g}: \overline{\mathcal{L}}_{1} \rightarrow \overline{\mathcal{L}}_{2}$ in $\operatorname{Sh}(X)$ is externally given by a locale map $g: \mathcal{L}_{1} \rightarrow \mathcal{L}_{2}$ in Sets such that $f_{2} \circ g=f_{1}$.

To see that this situation is relevant to our construction, first recall the Alexandrov topology on a poset $P$. Its opens are simply the upper sets, and the special upper sets of the form $U=\uparrow x$ form a basis of the Alexandrov topology. Equipping $P$ with the Alexandrov topology, one has an isomorphism of categories

$$
\text { Sets }^{P} \cong \operatorname{Sh}(P) \text {. }
$$

To understand this, just note that a sheaf $\bar{F}$ on $P$ is determined by its values on the basis opens $\uparrow x$; a functor $\underline{F}: P \rightarrow$ Sets then corresponds to $\bar{F}$ by

$$
\underline{F}(x)=\bar{F}(\uparrow x) .
$$

It is, then, immediate from (8) and (12) that

$$
\mathcal{T}(A) \cong \operatorname{Sh}(\mathcal{C}(A)),
$$

so that we have the above-mentioned external description of locales to our avail, with $X=\mathcal{C}(A)$.

Explicitly, to describe an internal locale $\mathcal{L}$ in $\operatorname{Sh}(X)$ externally, i.e. in terms of the topos Sets, consider the set $\Gamma \mathcal{O}(\mathcal{L})=\operatorname{Hom}_{\operatorname{Sh}(X)}(1, \mathcal{O}(\mathcal{L}))$ of global sections of the associated frame $\mathcal{O}(\mathcal{L})$; this set coincides with $\mathcal{O}(\mathcal{L})(X)$ (since a natural transformation in $\operatorname{Hom}_{\operatorname{Sh}(X)}(1, \mathcal{O}(\mathcal{L}))$ is determined by its value at $\left.X\right)$ and defines a frame $\mathcal{O}(\mathcal{L})(X)$ in Sets under the lattice structure borrowed from $\mathcal{O}(\mathcal{L})$. For $V \subseteq U$, let $\mathcal{L}_{V}^{U}: \mathcal{O}(\mathcal{L})(U) \rightarrow \mathcal{O}(\mathcal{L})(V)$ be the arrow part of the functor $\mathcal{O}(\mathcal{L}): \mathcal{O}(X)^{\mathrm{op}} \rightarrow$ Sets, with special case $\mathcal{L}_{V} \equiv \mathcal{L}_{V}^{X}$. The completeness of $\mathcal{O}(\mathcal{L})$ implies that $\mathcal{L}_{V}$ has a left adjoint $\mathcal{L}_{V}^{*}: \mathcal{O}(\mathcal{L})(V) \rightarrow \mathcal{O}(\mathcal{L})(X)$, which in turn defines a map $f^{*}: \mathcal{O}(X) \rightarrow \mathcal{O}(\mathcal{L})(X)$ by $f^{*}: V \mapsto \mathcal{L}_{V}^{*}\left(\top_{\mathcal{L}(V)}\right)$, where $\top_{\mathcal{L}(V)}$ is the top element of the lattice $\mathcal{O}(\mathcal{L})(V)$. This is a frame map, and if we write $\mathcal{O}(Y)=\mathcal{O}(\mathcal{L})(X)$, the corresponding locale map $f: Y \rightarrow X$ is the external description of $\mathcal{L}$.

Conversely, a locale $L$ in Sets along with a locale map $f: L \rightarrow X$ (i.e. a frame map $\left.f^{*}: \mathcal{O}(X) \rightarrow \mathcal{O}(L)\right)$ induces a locale $\mathcal{L}$ in $\operatorname{Sh}(X)$, defined as the sheaf $\mathcal{L}(U)=\{V \in$ $\left.L \mid V \leqslant f^{*}(U)\right\}$. These constructions are adjoint to each other, yielding an equivalence of the category $\operatorname{Loc}(\operatorname{Sh}(X))$ of locales in $\operatorname{Sh}(X)$ and the slice category Loc/ $X$ relative to the category Loc of locales in Sets.

For us, the external description of locales is useful for two reasons:

1. Returning to (9), we are clearly interested in the set

$$
\Gamma \mathcal{O}(\underline{\Sigma}) \cong \operatorname{Hom}_{\text {Sets }^{\mathcal{C}(A)}}(1, \mathcal{O}(\underline{\Sigma}))
$$

of opens in $\underline{\Sigma}$, as it encodes the quantum logic of our $\mathrm{C}^{*}$-algebra $A$. Let $\mathcal{O}(\bar{\Sigma})$ be the sheaf on $\mathcal{C}(A)$ that corresponds to $\mathcal{O}(\underline{\Sigma})$ by $(13)$, so that $\Gamma \mathcal{O}(\underline{\Sigma}) \cong \Gamma \mathcal{O}(\bar{\Sigma})$, with $\Gamma \mathcal{O}(\bar{\Sigma})=\operatorname{Hom}_{\operatorname{Sh}(\mathcal{C}(A))}(1, \mathcal{O}(\bar{\Sigma}))$. Clearly, if a poset $P$ has a bottom element 
$\perp$ and $Z$ is any object in $\operatorname{Sets}^{P}$, then $\Gamma Z \cong Z(\perp)$. This applies to $P=\mathcal{C}(A)$ with $\perp=\mathbb{C} \cdot 1$, so that

$$
\Gamma \mathcal{O}(\underline{\Sigma}) \cong \mathcal{O}(\underline{\Sigma})(\mathbb{C} \cdot 1) \cong \mathcal{O}(\bar{\Sigma})(\mathcal{C}(A))=\mathcal{O}(\Sigma)
$$

where we have used (11). Hence the external description of the quantum logic of the $\mathrm{C}^{*}$-algebra $A$ is entirely given in terms of the locale $\Sigma$ in Sets.

2. Important internal number systems in $\mathcal{T}(A)$ that are defined by geometric propositional theories $\mathbb{T}$ (see Subsect. 1.4) may be computed from (13) and their description in $\operatorname{Sh}(\mathcal{C}(A)$ ), which in turn is based on their external description in Sets. Specifically, if $[\mathbb{T}]$ is the locale defined by $\mathbb{T}$ in Sets, then the locale $[\overline{\mathbb{T}}] \equiv[\mathbb{T}]_{\operatorname{Sh}(X)}$ giving the interpretation of $\mathbb{T}$ in $\operatorname{Sh}(X)$ has external description $\pi_{1}: X \times[\mathbb{T}] \rightarrow X$, where $\pi_{1}$ is projection on the first component. It follows that the frame $\mathcal{O}([\overline{\mathbb{T}}])$ in $\operatorname{Sh}(X)$ corresponding to $[\overline{\mathbb{T}}]$ is given by the sheaf $U \mapsto \mathcal{O}(U \times[\mathbb{T}])$. Applying this to the case at hand, we see that the frame $\mathcal{O}([\mathbb{T}])$ corresponding to the interpretation $[\underline{\mathbb{T}}] \equiv[\mathbb{T}]_{\mathcal{C}(A)}$ of $\mathbb{T}$ in $\mathcal{T}(A)$ is given by the functor

$$
\mathcal{O}([\underline{\mathbb{T}}]): C \mapsto \mathcal{O}(\uparrow C \times[\mathbb{T}]) .
$$

See Subsects. 2.3 and 5.1 for examples of this procedure.

Our third example applies the second one to points of locales [63], and continues the discussion in Subsect. 1.4:

- Internally, a point of a locale $Y$ in a general topos $\mathcal{T}$ (internal to Sets for simplicity) is a locale map $* \rightarrow Y$, which is the same thing as an internal frame map $\mathcal{O}(Y) \rightarrow \Omega$ (where $\Omega$ is the subobject classifier in $\mathcal{T}$ ).

- Externally, we look at $\Omega$ as the frame $\operatorname{Sub}_{\mathcal{T}}(1)$ in Sets of subobjects of the terminal object 1 in $\mathcal{T}$. The locale in Sets with frame $\operatorname{Sub}_{\mathcal{T}}(1)$ is called the localic reflection $\operatorname{Loc}(\mathcal{T})$ of $\mathcal{T}$, i.e. $\mathcal{O}(\operatorname{Loc}(\mathcal{T}))=\operatorname{Sub}_{\mathcal{T}}(1)$. For example, in $\mathcal{T}=\operatorname{Sh}(X)$ one has $\operatorname{Sub}_{\operatorname{Sh}(X)}(1) \cong \mathcal{O}(X)$ and hence $\operatorname{Loc}(\operatorname{Sh}(X)) \cong X$. Applying the second example above, we find that the external description of the locale $*$ in $\operatorname{Sh}(X)$ is just id : $X \rightarrow X$, so that points in a locale $\mathcal{L}$ in $\operatorname{Sh}(X)$ with external description $f: Y \rightarrow X$ are given by locale maps $\varphi: X \rightarrow Y$ that satisfy $f \circ \varphi=\mathrm{id}$, i.e. cross-sections of $f$.

The fourth example continues both the previous one and the discussion of models in Subsect. 1.4. We initially defined a standard model of a geometric propositional theory $\mathbb{T}$ as a locale map $* \rightarrow[\mathbb{T}]$, and subsequently mentioned more general models $Y \rightarrow[\mathbb{T}]$, still in Sets. We now consider even more general models of $\mathbb{T}$ in a topos $\mathcal{T}$.

- Externally, these are given by locale maps $\operatorname{Loc}(\mathcal{T}) \rightarrow[\mathbb{T}]$ in Sets. This is because the classifying topos of $\mathbb{T}$ is $\mathrm{Sh}([\mathbb{T}])$, and one has an equivalence between geometric morphisms $\mathcal{T} \rightarrow \operatorname{Sh}([\mathbb{T}]$ ) (which classify $\mathbb{T}$-models in $\mathcal{T}, c f$. [63, Thm. X.6.1]) and locale maps $\operatorname{Loc}(\mathcal{T}) \rightarrow[\mathbb{T}]($ see $[63, \operatorname{Sect}$. IX.5]).

- Internally, one may interpret the theory $\mathbb{T}$ in $\mathcal{T}$ and thus define a locale $[\mathbb{T}]_{\mathcal{T}}$ internal to $\mathcal{T}$. The points of this locale, i.e. the locale maps $* \rightarrow[\mathbb{T}]_{\mathcal{T}}$ or frame maps $\mathcal{O}\left([\mathbb{T}]_{\mathcal{T}}\right) \rightarrow \Omega_{\mathcal{T}}$, describe the models of $\mathbb{T}$ in $\mathcal{T}$ internally.

One may explicitly verify the equivalence between the internal and the external descriptions for $\mathcal{T}=\operatorname{Sh}(X)$, for in that case the external description of $[\mathbb{T}]_{\mathcal{T}}$ is the map $\pi_{1}: X \times[\mathbb{T}] \rightarrow X$. Hence locale maps $\varphi: X \rightarrow X \times[\mathbb{T}]$ that satisfy $\pi_{1} \circ \varphi=$ id are just (unconstrained) locale maps $X \rightarrow[\mathbb{T}]$. 
1.8. Observation and approximation. Our construction of the locale map $\delta(a): \underline{\Sigma} \rightarrow$ $\underline{\mathbb{R}}$ in Sect. 1.6 involves the so-called interval domain $\mathbb{I R}[74]$. To motivate its definition, consider the approximation of real numbers by nested intervals with endpoints in $\mathbb{Q}$. For example, the real number $\pi$ can be described by specifying the sequence

$$
[3,4],[3.1,3.2],[3.14,3.15],[3.141,3.142], \ldots
$$

Each individual interval may be interpreted as finitary information about the real number under scrutiny, involving the single observation that the real number is contained in the interval. This description of the reals, which goes back to L.E.J. Brouwer, is formalized by the notion of the interval domain. Consider the poset $\mathbb{I} \mathbb{R}$ whose elements are compact intervals $[a, b]$ in $\mathbb{R}$ (including singletons $[a, a]=\{a\}$ ), ordered by reverse inclusion (for a smaller interval means that we have more information about the real number that the ever smaller intervals converge to). This poset is a so-called dcpo (directed complete partial order); directed suprema are simply intersections. As such, it carries the Scott topology [2,74], whose closed sets are lower sets that are closed under suprema of directed subsets. Here a lower set in a poset $P$ is a subset $L \subseteq P$ such that $x \in L$ and $y \leqslant x$ implies $y \in L$; equivalently, $\downarrow L \subseteq L$, where $\downarrow L=\{y \in P \mid \exists x \in L: y \leqslant x\}$. (Lower sets are sometimes called down sets or downward closed sets.) Consequently, Scott opens must be upper sets $U$ (defined in the obvious way) with the additional property that for every directed set $D$ with $\bigvee D \in U$ the intersection $D \cap U$ is nonempty. In the case of $\mathbb{I}$, this means that each open interval $(p, q)$ in $\mathbb{R}$ (with $p=-\infty$ and $q=+\infty$ allowed) corresponds to a Scott open $\{[a, b] \mid p<a, b<q\}$ in $\mathbb{I}$, and these opens form a basis of the Scott topology. The collection $\mathcal{O}_{\text {Scott }}(\mathbb{I} \mathbb{R})$ is, of course, a frame, initially defined in Sets. The basis opens $(r, s)$ may be reinterpreted as a collection of generators for this frame, which from the point of view of generators and relations differs from the frame $\mathcal{O}(\mathbb{R})$ of Dedekind reals in that the relation $(p, q)=\left(p, q_{1}\right) \vee\left(p_{1}, q\right)$ for $p \leqslant p_{1} \leqslant q_{1} \leqslant q$ holds for the reals, but not for the interval domain (see [51, D4.7.4] or Subsect. 2.3 below for the other relations for $\mathcal{O}(\mathbb{R})$ ). The interval domain admits an internal definition in any topos. Its realization in $\mathcal{T}(A)$ will play an important role in this paper; see Subsect. 5.1.

A related notion of approximation appears when considering an observable $a \in A_{\text {sa }}$ of a quantum system described by a $\mathrm{C}^{*}$-algebra $A$, as seen from inside its associated topos $\mathcal{T}(A)$. Specifically, we should approximate $a$ within each classical snapshot $C$ of $A$, where $C \in \mathcal{C}(A)$ is some commutative subalgebra. The difficulty is, of course, that $a$ need not lie in $C$, but neither is there a single element of $C$ that forms the 'best approximation' of $a$ in $C$. The best one can do is approximate $a$ by a family of elements of $C$, as follows.

The self-adjoint part $A_{\mathrm{sa}}$ of a $\mathrm{C}^{*}$-algebra $A$ has a natural partial order $\leqslant$, defined by $a \leqslant b$ iff $b-a=c^{*} c$ for some $c \in A$. (Equivalently, $a \leqslant b$ iff $b-a=f^{2}$ for some $f \in A_{\text {sa }}$.) This partial order is linear-in the sense that $a+c \leqslant b+c$ whenever $a \leqslant b$. For the $\mathrm{C}^{*}$-algebra $A=C(X, \mathbb{C})$ one just recovers the pointwise order on (real-valued) functions, since $A_{\mathrm{sa}}=C(X, \mathbb{R})$. For $A=B(H)$, the bounded operator on Hilbert space $H$, one has $a \geq 0$ for $a \in A_{\text {sa }}$ iff $(\Psi, a \Psi) \geq 0$ for all $\Psi \in H$. (Indeed, this is really a pointwise order as well, if one regards operators $a$ on $H$ as functions $\hat{a}$ on $H$ by $\hat{a}(\Psi)=(\Psi, a \Psi)$. See e.g.[58].) Thus one may approximate $a$ in $C$ by collections of intervals of the type $\left\{[f, g] \mid f, g \in C_{\mathrm{sa}}, f \leqslant a \leqslant g\right\}$ (note that this is inhabited, since $f=-\|a\| \cdot 1$ and $g=\|a\| \cdot 1$ occur). Since the intervals $[f, g]$ in $C_{\text {sa }}$ fail to form a dcpo, however, a slight adaptation of this idea is needed, for which we refer to Subsect. 5.1 below. 
1.9. Structure of this article. Section 2 reviews the Gelfand duality theory in a topos due to Banaschewski and Mulvey. Our original results are as follows:

1. The construction of the 'quantum phase space' $\underline{\Sigma}(A)$ from a $C^{*}$-algebra $A$ in Sets as the Gelfand spectrum of the Bohrification $\underline{A}$ of $A$ in the topos $\mathcal{T}(A)$ is explained in Sect. 3. This section also contains our version of the Kochen-Specker Theorem.

2. Section 4 first describes the construction of states on $A$ as probability integrals on the self-adjoint part of $\underline{A}$. These, in turn, are equivalent to probability valuations on its Gelfand spectrum $\underline{\Sigma}(\underline{A})$. On this basis, we eventually show that states define subobjects of the quantum phase space $\underline{\Sigma}(\underline{A})$, as in classical physics (see (3)).

3. The interpretation of observables in $A$ in terms of the Bohrification $\underline{A}$ is the subject of Sect. 5. In particular, we give our analogue of the Daseinisation map of Döring and Isham in Subsect. 5.2 (and more fully in Appendix A.2).

4. The pairing of states and propositions is elucidated in Sect. 6, yielding an element of the subobject classifier of $\mathcal{T}(A)$ that we explicitly compute. This pairing connects the mathematical constructions to quantum physics and completes Steps 1 to 5 of our general program mentioned at the beginning of Subsect. 1.2.

Appendix A contains a number of technical results that somewhat distract from the main development of the paper. Finally, Appendix B discusses related work by Döring and Isham, which partly inspired the present article.

\section{C*-Algebras and Gelfand Duality in a Topos}

This section recapitulates a constructive version of Gelfand duality, which is valid in every topos [5-7]. Recall that the usual version of Gelfand duality characterises unital commutative $\mathrm{C}^{*}$-algebras as algebras of complex-valued continuous functions on a compact Hausdorff space. More generally, the category cCStar of unital commutative $\mathrm{C}^{*}$-algebras and unital *-homomorphisms is dual to the category KHausSp of compact Hausdorff spaces and continuous maps (see [48] for a proof aimed at algebraists and [59] for a proof in the spirit of $\mathrm{C}^{*}$-algebras). From a topos-theoretic point of view, this formulation is internal to the topos Sets, since both categories are defined relative to it.

To understand the generalization of Gelfand duality to arbitrary topoi, a slight reformulation of the situation in Sets is appropriate: we replace topological spaces $X$ by the associated locales, and hence replace KHausSp by the equivalent category KRegLoc of compact regular locales [48]. ${ }^{2}$ Consequently, the duality cCStar $\simeq$ KHausSp may be replaced by cCStar $\simeq$ KRegLoc: the contravariant functor cCStar $\rightarrow$ KRegLoc is still given by $A \mapsto \Sigma(A)$, where $\Sigma(A)$ is the locale defined by the usual Gelfand spectrum of $A$ (i.e. its pure state space), and in the opposite direction one has the familiar expression $X \mapsto C(X, \mathbb{C})$, in which the right-hand side now stands for the locale maps from $X$ to $\mathbb{C}$.

For technical reasons, in general topoi regular compact locales have to be replaced by completely regular compact locales, ${ }^{3}$ but otherwise one has a direct generalization of

${ }^{2}$ A locale $\mathcal{L}$ is compact if every subset $S \subseteq \mathcal{L}$ with $\bigvee S=\top$ has a finite subset $F$ with $\bigvee F=\top$. It is regular if every element of $\mathcal{L}$ is the join of the elements well inside itself, where $a$ is well inside $b$ (denoted $a \ll b$ ) if there exists $c$ with $c \wedge a=\perp$ and $c \vee b=\top$. The (internal) categories KRegLoc and KHausSp in a topos $\mathcal{T}$ are equivalent when the full axiom of choice is available in $\mathcal{T}$ [48].

${ }^{3}$ See [48 or 7] for the definition of complete regularity. If the axiom of dependent choice (stating that for any nonempty set $X$ and any relation $R \subseteq X \times X$ such that for all $x$ there is an $y$ with $(x, y) \in R$, there is a sequence $\left(x_{n}\right)$ such that $\left(x_{n}, x_{n+1}\right) \in R$ for each $n \in \mathbb{N}$ ) is valid in a topos, then compact regular locales are automatically completely regular. This is the case in Sets, for example (where, of course, the full axiom of choice holds), and also in topoi like $\mathcal{T}(A)$ consisting of functors whose codomain validates dependent choice [41]. 
the above reformulation of Gelfand duality in Sets. The following theorem is predicated on an internal definition of the category cCStar, which we shall give in Subsect. 2.1. Here and in what follows, all mathematical symbols are to be interpreted in the internal language of the topos $\mathcal{T}$ at hand.

Theorem 1 (Gelfand duality in a topos) [5-7]. In any topos $\mathcal{T}$, there is a categorical duality (i.e. contravariant equivalence)

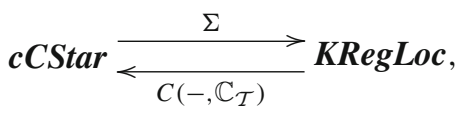

where the categories in question are defined internally to $\mathcal{T}$.

For $A \in \mathbf{c C S t a r}$, the locale $\Sigma(A)$ is called the Gelfand spectrum of $A$. Here the symbol $\mathbb{C}_{\mathcal{T}}$ stands for the locale of Dedekind complex numbers in $\mathcal{T}$.

2.1. $C^{*}$-algebras in a topos. In any topos $\mathcal{T}$ (with natural numbers object), the rationals $\mathbb{Q}$ can be interpreted [63, Sect. VI.8], as can the Gaussian integers $\mathbb{C}_{\mathbb{Q}}=\{p+q i: p, q \in$ $\mathbb{Q}\}$. For example, the interpretation of $\mathbb{C}_{\mathbb{Q}}$ in a functor topos Sets ${ }^{\mathbf{C}}$ (where, in our case, $\mathbf{C}$ is a poset) is the constant functor that assigns the set $\mathbb{C}_{\mathbb{Q}}$ to every $C \in \mathbf{C}$.

A *algebra in $\mathcal{T}$ is a vector space $A$ over $\mathbb{C}_{\mathbb{Q}}$ that carries an associative bilinear map $\therefore A \times A \rightarrow A$, and is furthermore equipped with a map $(-)^{*}: A \rightarrow A$ satisfying

$$
(a+b)^{*}=a^{*}+b^{*}, \quad(z \cdot a)^{*}=\bar{z} \cdot a^{*}, \quad(a \cdot b)^{*}=b^{*} \cdot a^{*}, \quad a^{* *}=a,
$$

for all $a, b \in A$ and $z \in \mathbb{C}_{\mathbb{Q}} \cdot A$ is called commutative if $a \cdot b=b \cdot a$ for all $a, b \in A$, and unital if there is a neutral element 1 for the multiplication.

To define an internal $C^{*}$-algebra, we define a seminorm on such an algebra; in general, a norm may not actually be definable in the internal language of a topos. This is a relation $N \subseteq A \times \mathbb{Q}^{+}$, which in Sets would have the meaning that $(a, q) \in N$ iff $\|a\|<q$. In general, $N$ must satisfy

$$
\begin{aligned}
& (0, p) \in N, \\
& \exists_{q \in \mathbb{Q}^{+}}[(a, q) \in N], \\
(a, p) \in N \rightarrow & \left(a^{*}, p\right) \in N, \\
(a, q) \in N \leftrightarrow \exists_{p<q}[(a, p) \in N], & (a+b, p+q) \in N, \\
(a, p) \in N \wedge(b, q) \in N \rightarrow & (a \cdot b, p \cdot q) \in N, \\
(a, p) \in N \wedge(b, q) \in N \rightarrow & (z \cdot a, p \cdot q) \in N \\
& (a, p) \in N \rightarrow \quad(|z|<q),
\end{aligned}
$$

for all $a, b \in A, p, q \in \mathbb{Q}^{+}$, and $z \in \mathbb{C}_{\mathbb{Q}}$. For a unital $*_{\text {-algebra, we also require }}$

$$
(1, p) \in N \quad(p>1) \text {. }
$$

If the seminorm relation furthermore satisfies

$$
\left(a^{*} \cdot a, q^{2}\right) \in N \leftrightarrow(a, q) \in N
$$

for all $a \in A$ and $q \in \mathbb{Q}^{+}$, then $A$ is said to be a pre-semi-C*-algebra. 
To proceed to a $C^{*}$-algebra, one requires $a=0$ whenever $(a, q) \in N$ for all $q$ in $\mathbb{Q}^{+}$, making the seminorm into a norm, and subsequently one requires this normed space to be complete in a suitable sense (see [7] for details). As a consequence of its completeness, a $C^{*}$-algebra is automatically an algebra over a suitable completion of $\mathbb{C}_{\mathbb{Q}}$ (and not just over $\mathbb{C}_{\mathbb{Q}}$ itself, as baked into the definition). Note that in general topoi one has to distinguish certain real and complex number objects that coincide in Sets. From $\mathbb{Q}$, one may construct the locale $\mathbb{R}_{d} \equiv \mathbb{R}$ of Dedekind real numbers [63, Sect. VI.8] (see also Subsect. 2.3 below); we will drop the suffix $d$ for simplicity. The object $\operatorname{Pt}(\mathbb{C})$ (which is the completion of $\mathbb{C}_{\mathbb{Q}}$ meant above) comprises the points of the complexified locale $\mathbb{C}=\mathbb{R}+i \mathbb{R}$; see also [7] for a direct description that avoids $\mathbb{R}$. In Sets, $\mathbb{C}$ is the locale with frame $\mathcal{O}(\mathbb{C})$, where (abusing notation) $\mathbb{C}$ are the usual complex numbers. In any topos, the one-dimensional $\mathrm{C}^{*}$-algebra $C(*, \mathbb{C})$ is nothing but $\operatorname{Pt}(\mathbb{C})$ and has Gelfand spectrum $*$ (i.e. the locale with frame $\Omega$ ).

A unital *-homomorphism between $C^{*}$-algebras $A$ and $B$ is, as usual, a linear map $f: A \rightarrow B$ satisfying $f(a b)=f(a) f(b), f\left(a^{*}\right)=f(a)^{*}$ and $f\left(1_{A}\right)=1_{B}$. Unital $\mathrm{C}^{*}$-algebras with unital *-homomorphisms form a category CStar (internal to $\mathcal{T}$ ); commutative unital $\mathrm{C}^{*}$-algebras form a full subcategory cCStar thereof.

2.2. Spectrum. The definition of the category KRegLoc of completely regular compact locales can be internalized without difficulty. The next step is to explicitly describe the Gelfand spectrum $\Sigma(A) \equiv \Sigma$ of a given commutative $\mathrm{C}^{*}$-algebra $A$. We will do so following the reformulation in [27,29] of the pioneering work of Banaschewski and Mulvey [7].

To motivate the description, note that even in Sets the spectrum is now described (with the usual notational ambiguity explained in Subsect. 1.3) as the locale $\Sigma$ defined by the frame $\mathcal{O}(\Sigma)$ of open subsets of the usual Gelfand spectrum $\Sigma$ of $A$ (defined as the subset of the dual $A^{*}$ consisting of space of nonzero multiplicative functionals on $A$ in the relative weak* topology). The topology on the space $\Sigma$ can be described by giving a sub-base, for which one often takes $U_{\left(a, \rho_{0}, \varepsilon\right)}=\left\{\rho|| \rho(a)-\rho_{0}(a) \mid<\varepsilon\right\}$ for $a \in A, \rho_{0} \in \Sigma, \varepsilon>0$. However, a much simpler choice of sub-base would be

$$
\mathrm{D}_{a}=\{\rho \in \Sigma \mid \rho(a)>0\},
$$

where $a \in A_{\mathrm{sa}}$. Both the property that the $\rho$ are multiplicative and the fact that the $\mathrm{D}_{a}$ form a sub-base of the Gelfand topology may then be expressed lattice-theoretically by saying that $\mathcal{O}(\Sigma)$ is the frame $F_{A_{\mathrm{sa}}}$ freely generated by the formal symbols $\mathrm{D}_{a}, a \in A_{\mathrm{sa}}$, subject to the relations

$$
\begin{aligned}
\mathrm{D}_{1} & =\top \\
\mathrm{D}_{a} \wedge \mathrm{D}_{-a} & =\perp \\
\mathrm{D}_{-b} & =\perp \\
\mathrm{D}_{a+b} & \leqslant \mathrm{D}_{a} \vee \mathrm{D}_{b}, \\
\mathrm{D}_{a b} & =\left(\mathrm{D}_{a} \wedge \mathrm{D}_{b}\right) \vee\left(\mathrm{D}_{-a} \wedge \mathrm{D}_{-b}\right),
\end{aligned}
$$

supplemented with the 'regularity rule'

$$
\mathrm{D}_{a} \leqslant \bigvee_{r \in \mathbb{Q}^{+}} \mathrm{D}_{a-r} .
$$


This turns out to be a correct description of the spectrum of $A$ also in an arbitrary topos $\mathcal{T}$, in which case (18)-(23) have to be interpreted in $\mathcal{T}$, of course. ${ }^{4}$

2.3. Gelfand transform. Classically, for a commutative unital $\mathrm{C}^{*}$-algebra $A$ the Gelfand transform $A \stackrel{\cong}{\cong} C(\Sigma, \mathbb{C})$ is given by $a \mapsto \hat{a}$ with $\hat{a}(\rho)=\rho(a)$. In our setting it is convenient to restrict the Gelfand transform to $A_{\mathrm{sa}}$, yielding an isomorphism

$$
A_{\mathrm{sa}} \cong C(\Sigma, \mathbb{R}) \text {. }
$$

In a topos $\mathcal{T}$, the Gelfand transform of an internal commutative unital $\mathrm{C}^{*}$-algebra $A$ in $\mathcal{T}$ associates a locale map

$$
\hat{a}: \Sigma \rightarrow \mathbb{R}_{\mathcal{T}}
$$

to each $a \in A_{\text {sa }}$, where $\Sigma$ is the spectrum of $A$ and $\mathbb{R}_{\mathcal{T}}$ is the locale of internal Dedekind real numbers in $\mathcal{T}$; see below. Recalling from Subsect. 1.3 that $\hat{a}$ is by definition a frame map

$$
\hat{a}^{-1}: \mathcal{O}\left(\mathbb{R}_{\mathcal{T}}\right) \rightarrow \mathcal{O}(\Sigma)
$$

and using the " $\lambda$-conversion rule" $\frac{Y \rightarrow Z^{X}}{Y \times X \rightarrow Z}$ [63, Sect. I.6], we note that the Gelfand transform may alternatively be regarded as a map

$$
\hat{\imath}: A_{\mathrm{sa}} \times \mathcal{O}\left(\mathbb{R}_{\mathcal{T}}\right) \rightarrow \mathcal{O}(\Sigma) .
$$

Thus the use of the symbol $a \in A_{\text {sa }}$ in the internal language of $\mathcal{T}$ may be avoided in principle. In practice, however, we will often use the notation (25) or (26), and hence the formal symbols $D_{a}$. For example, in the description (18)-(23) of the spectrum $\Sigma$ in terms of generators and relations, it is sufficient to define the frame map (26) on basic opens $(-\infty, r)$ and $(s, \infty)$ in $\mathbb{R}_{\mathcal{T}}$. In the classical case (i.e. in Sets) discussed above, one has $\hat{a}^{-1}(0, \infty)=\mathrm{D}_{a}$ from $(17)$, and this remains true in general if $\hat{a}^{-1}$ has the meaning (26). Using (18)-(21), one then finds

$$
\begin{aligned}
\hat{a}^{-1}:(-\infty, s) & \mapsto \mathrm{D}_{s-a} ; \\
(r, \infty) & \mapsto \mathrm{D}_{a-r} .
\end{aligned}
$$

As $\hat{a}^{-1}$ is a frame map, for bounded open intervals $(r, s)$ we therefore obtain ${ }^{5}$

$$
\hat{a}^{-1}:(r, s) \mapsto \mathrm{D}_{s-a} \wedge \mathrm{D}_{a-r} .
$$

We now recall an explicit construction of the Dedekind reals [40,51, D4.7.4 \& D4.7.5]. Define the propositional geometric theory $\mathbb{T}_{\mathbb{R}}$ generated by formal symbols $(p, q) \in$ $\mathbb{Q} \times \mathbb{Q}$ with $p<q$, ordered as $(p, q) \leqslant\left(p^{\prime}, q^{\prime}\right)$ iff $p^{\prime} \leqslant p$ and $q \leqslant q^{\prime}$, subject to the following axioms (or relations):

4 See [77] and the Appendix to this paper for the procedure of constructing a frame from generators and relations. Equivalently, in the spirit of [7] one could rephrase the above definition by saying that $\Sigma$ is the locale $[\mathbb{T}]$ corresponding to the propositional geometric theory $\mathbb{T}$ (in the sense explained in Subsect. 1.4) determined by the collection of propositions $\mathrm{D}_{a}, a \in A_{\text {sa }}$, subject to the axioms (18)-(23), with $\leqslant$ replaced by $\vdash$.

5 Banaschewski and Mulvey [7] work with such intervals $(r, s)$ as basic opens, in terms of which they write the Gelfand transform as $\hat{a}^{-1}:(r, s) \mapsto a \in(r, s)$. Here the role of generators of the locale $\Sigma$ is played by elementary propositions of the logical theory generating $\Sigma$ as its Lindenbaum algebra, our generator $\mathrm{D}_{a}$ corresponding to their proposition $a \in(0, \infty)$. Classically, the proposition $a \in(r, s)$ may be identified with the open $a^{-1}(r, s)$ in the spectrum $\Sigma ; c f$. Subsect. 1.1 . 
1. $\left(p_{1}, q_{1}\right) \wedge\left(p_{2}, q_{2}\right)=\left(\max \left\{p_{1}, p_{2}\right\}, \min \left\{q_{1}, q_{2}\right\}\right)$ if $\max \left\{p_{1}, p_{2}\right\}<\min \left\{q_{1}, q_{2}\right\}$, and $\left(p_{1}, q_{1}\right) \wedge\left(p_{2}, q_{2}\right)=\perp$ otherwise;

2. $(p, q)=\bigvee\left\{\left(p^{\prime}, q^{\prime}\right) \mid p<p^{\prime}<q^{\prime}<q\right\}$;

3. $\mathrm{\top}=\bigvee\{(p, q) \mid p<q\}$;

4. $(p, q)=\left(p, q_{1}\right) \vee\left(p_{1}, q\right)$ if $p \leqslant p_{1} \leqslant q_{1} \leqslant q$.

This theory may be interpreted in any topos $\mathcal{T}$, defining an internal locale $\left(\mathbb{T}_{\mathbb{R}}\right)_{\mathcal{T}} \equiv \mathbb{R}_{\mathcal{T}}$ with associated frame $\mathcal{O}\left(\mathbb{R}_{\mathcal{T}}\right)$. Points $m$ of $\mathbb{R}_{\mathcal{T}}$, i.e. frame maps $m^{-1}: \mathcal{O}\left(\mathbb{R}_{\mathcal{T}}\right) \rightarrow \Omega_{\mathcal{T}}$, correspond bijectively to Dedekind cuts $(L, U)$ of $\mathbb{Q}(c f .[63$, p. 321]) in the following way: a model $m$ determines a Dedekind cut by

$$
\begin{aligned}
L & =\{p \in \mathbb{Q} \mid m \models(p, \infty)\}, \\
U & =\{q \in \mathbb{Q} \mid m \models(-\infty, q)\},
\end{aligned}
$$

where $(p, \infty)$ and $(-\infty, q)$ are defined in terms of the formal generators of the frame $\mathcal{O}(\mathbb{Q})$ by $(p, \infty)=\bigvee\{(p, r) \mid p<r\}$ and $(-\infty, q)=\bigvee\{(r, q) \mid r<q\}$. The notation $m \models(p, q)$ used here means that $m^{-1}(p, q)=\top$, where $\top: 1 \rightarrow \Omega_{\mathcal{T}}$ is the truth element of $\Omega_{\mathcal{T}}$ and $(p, q)$ is seen as an arrow $(p, q): 1 \rightarrow \mathbb{Q} \times \mathbb{Q} \rightarrow \mathcal{O}\left(\mathbb{R}_{\mathcal{T}}\right)$. Conversely, a Dedekind cut $(L, U)$ uniquely determines a point $m$ that maps a generator $I=(p, q)$ to $m(I)=\top$ iff $I \cap U \neq \emptyset$ and $I \cap L \neq \emptyset$. The Dedekind reals $\operatorname{Pt}\left(\mathbb{R}_{\mathcal{T}}\right)$, then, are defined in any topos $\mathcal{T}$ as the subobject of $\mathcal{P}\left(\mathbb{Q}_{\mathcal{T}}\right) \times \mathcal{P}\left(\mathbb{Q}_{\mathcal{T}}\right)$ consisting of those $(L, U)$ that are points of $\mathbb{R}_{\mathcal{T}}$ [63].

We mention four examples:

1. In $\mathcal{T}=$ Sets, a point $m$ of $\mathbb{R} \equiv \mathbb{R}_{\text {Sets }}$ corresponds to a real $x$ described in the usual calculus way, so that $L=\{p \in \mathbb{Q} \mid p<x\}$ and $U=\{q \in \mathbb{Q} \mid x>q\}$. Hence $\operatorname{Pt}(\mathbb{R})$ may be identified with $\mathbb{R}$ in the usual sense, and $\mathbb{R}$ is spatial as a locale; its frame $\mathcal{O}(\mathbb{R})$ is just the usual topology of $\mathbb{R}[51, \mathrm{D} 4.7 .4]$. From this perspective, the first condition in the definition of $\mathbb{T}_{\mathbb{R}}$ enforces that $L$ and $U$ are lower and upper sections of $\mathbb{Q}$, respectively, the second implies that they are open, and the third means that $L$ and $U$ are both inhabited. The fourth - Dedekind - relation says that $L$ and $U$ 'kiss' each other. ${ }^{6}$

2. If $X$ is a topological space (or, more generally, a locale), the structure of the locale $\mathbb{R}_{\operatorname{Sh}(X)}$ and its associated sheaf of Dedekind reals $\operatorname{Pt}\left(\mathbb{R}_{\operatorname{Sh}(X)}\right)$ in the topos $\operatorname{Sh}(X)$ of sheaves on $X$ follows from the argument above (16) in Subsect. 1.7. First, the frame of Dedekind reals is given by the sheaf

$$
\mathcal{O}(\mathbb{R})_{\operatorname{Sh}(X)}: U \mapsto \mathcal{O}(U \times \mathbb{R}),
$$

whereas the Dedekind real numbers object is the sheaf (see also [63])

$$
\operatorname{Pt}(\mathbb{R})_{\operatorname{Sh}(X)}: U \mapsto C(U, \mathbb{R}) .
$$

3. Consequently, using (12) and (13) we infer that in our functor topos $\mathcal{T}(A)=$ Sets $^{\mathcal{C}(A)}$, the frame of Dedekind reals is the functor

$$
\mathcal{O}(\underline{\mathbb{R}}): C \mapsto \mathcal{O}((\uparrow C) \times \mathbb{R})
$$

\footnotetext{
6 The collection of $L$ satisfying only the first three relations forms the locale of lower reals, which we denote by $\mathbb{R}_{l}$. Locale maps to $\mathbb{R}_{l}$ are, classically, lower-semicontinuous real-valued functions. Analogously, there is a locale $\mathbb{R}_{u}$ of upper reals. See [51].
} 
the set on the right-hand side may be identified with the set of monotone functions from $\uparrow C$ to $\mathcal{O}(\mathbb{R}) .^{7}$

Perhaps surprisingly, the associated functor of points $\operatorname{Pt}(\underline{\mathbb{R}})$ may be identified with the constant functor

$$
\operatorname{Pt}(\underline{\mathbb{R}}): C \mapsto \mathbb{R}
$$

this follows from (34) and the fact that Alexandrov-continuous functions $U \rightarrow \mathbb{R}$ (or, indeed, into any Hausdorff space) must be locally constant on any open $U \subseteq \mathcal{C}(A){ }^{8}$

4. If $\Sigma$ is the Gelfand spectrum of a commutative $C^{*}$-algebra $A$ in $\mathcal{T}$, in the sheaf topos $\operatorname{Sh}(\Sigma)$ internal to $\mathcal{T}$ we similarly have

$$
\operatorname{Pt}(\mathbb{R})_{\operatorname{Sh}(\Sigma)}: U \mapsto C(U, \mathbb{R})
$$

Here we identify the open $U$ of $\Sigma$ with its associated sublocale $\{V \in \Sigma \mid V \leqslant U\}$ of $\Sigma$. This locale, as well as $\mathbb{R}$, is to be interpreted in the ambient topos $\mathcal{T}$ as explained in the above items.

Example 4 leads to an elegant reformulation of the isomorphism (24) given by the Gelfand theory: since

$$
C(\Sigma, \mathbb{R})=\Gamma\left(\operatorname{Pt}(\mathbb{R})_{\operatorname{Sh}(\Sigma)}\right)
$$

where $\Gamma$ is the global sections functor, one infers from (24) that

$$
A_{\mathrm{sa}} \cong \Gamma\left(\operatorname{Pt}(\mathbb{R})_{\operatorname{Sh}(\Sigma)}\right)
$$

In other words, the self-adjoint part of a unital commutative $\mathrm{C}^{*}$-algebra $A$ in a topos is isomorphic to the global sections of the Dedekind reals in the internal topos of sheaves on its spectrum (and $A$ itself "is" the complex numbers in the same sense).

\section{The Internal C*-Algebra and its Spectrum}

In this section we explain the association of a particular commutative $\mathrm{C}^{*}$-algebra $\underline{A}$, which is internal to a certain functor topos $\mathcal{T}(A)$, to a (generally) noncommutative $\mathrm{C}^{*}$-algebra $A$. As mentioned in the Introduction, this construction is motivated by Bohr's doctrine of classical concepts, so that we call $\underline{A}$ the Bohrification of $A$.

7 This identification proceeds in two steps. First, for any topological space $X$ one has a bijection $\mathcal{O}(X) \cong$ $C(X, S)$, where $S=\{0,1\}$ carries the Sierpinski topology, see Subsect. 1.4; explicitly, $U \in \mathcal{O}(X)$ is mapped to $\chi_{U}$, whereas in the opposite direction $g \in C(X, S)$ is sent to $g^{-1}(\{1\})$. Hence $\mathcal{O}(\uparrow C \times \mathbb{R}) \cong C(\uparrow C \times \mathbb{R}, S)$ (with apologies for the double use of $C$, first for 'continuous' and second for $C \in \mathcal{C}(A)$ ). Second, in general $\lambda$-conversion or 'currying' gives a bijection between functions $Y \times \mathbb{R} \rightarrow S$ and functions $Y \rightarrow S^{\mathbb{R}}$; with $Y=\uparrow C$ equipped with the Alexandrov topology and $C(\mathbb{R}, S) \cong \mathcal{O}(\mathbb{R})$, continuity then translates into monotonicity.

${ }^{8}$ We take $X=\mathcal{C}(A)$, equipped with the Alexandrov topology, and prove that in this topology any $f \in C(U, \mathbb{R})$ must be locally constant. Suppose $C \leqslant D$ in $U$, take and $V \subseteq \mathbb{R}$ open with $f(C) \in V$. Then tautologically $C \in f^{-1}(V)$ and $f^{-1}(V)$ is open by continuity of $f$. But the smallest open set containing $C$ is $\uparrow C$, which contains $D$, so that $f(D) \in V$. Taking $V=(f(C)-\epsilon, \infty)$ gives $f(D)>f(C)-\epsilon$ for all $\epsilon>0$, whence $f(D) \geqslant f(C)$, whereas $V=(-\infty, f(C)+\epsilon)$ yields $f(D) \leqslant f(C)$. Hence $f(C)=f(D)$. 
3.1. The topos associated to a $C^{*}$-algebra. We first construct the topos $\mathcal{T}(A)$ in which $A$ resides and draw attention to the functoriality of the map $A \mapsto \mathcal{T}(A)$. We denote the category of partially ordered sets and monotone functions by Poset.

Proposition 2. There is a functor $\mathcal{C}:$ CStar $\rightarrow$ Poset, defined on objects as

$$
\mathcal{C}(A)=\{C \subseteq A \mid C \in c \text { CStar }\}
$$

ordered by inclusion. On a morphism $f: A \rightarrow B$ of CStar, it acts as $\mathcal{C}(f): \mathcal{C}(A) \rightarrow$ $\mathcal{C}(B)$ by the direct image $C \mapsto f(C)$.

As announced in (8) in the Introduction, the collection of functors $\mathcal{C}(A) \rightarrow$ Sets forms a topos $\mathcal{T}(A)=\operatorname{Sets}^{\mathcal{C}(A)}$. This is the topos associated to $A$. We recall our convention to underline entities internal to $\mathcal{T}(A)$. The subobject classifier $\underline{\Omega}$ in $\mathcal{T}(A)$ has already been given in (10).

Recall that a geometric morphism $f: \mathcal{S} \rightarrow \mathcal{T}$ between topoi is a pair of adjoint functors, consisting of a direct image part $f_{*}: \mathcal{S} \rightarrow \mathcal{T}$ and an inverse image part $f^{*}: \mathcal{T} \rightarrow \mathcal{S}$, of which $f^{*}$ is required to preserve finite limits. Denote the category of elementary topoi and geometric morphisms by Topos. ${ }^{9}$

Proposition 3. There is a functor $\mathcal{T}:$ CStar $\rightarrow$ Topos, defined on objects by $\mathcal{T}(A)=$ Sets $^{\mathcal{C}}{ }^{(A)}$, the category of functors from $\mathcal{C}(A)$ to the ambient topos.

This immediately follows from Theorem VII.2.2 in [63] (p. 359) and Proposition 2.

To close this subsection, note that instead of initially regarding $\mathcal{C}(A)$ as a poset as in the main text, we could have considered it as a category from the start, having the same objects, but with (equivalence classes of) monomorphisms as arrows (instead of inclusions). The functor in Proposition 2 would then have the category Cat of categories as its codomain. This would still have allowed us to define the associated topos, and also the internal $\mathrm{C}^{*}$-algebra we will define below. From then on, most constructions will be within the associated topos, and hence go through as well.

3.2. Bohrification. Whereas the previous subsection considered the topos $\mathcal{T}(A)$ associated to a $C^{*}$-algebra $A$, this one is devoted to a particular object $\underline{A}$ in this topos. In fact, the definition of $\underline{A}$ is 'tautological' in a literal sense.

Definition 4. Let $A$ be a $C^{*}$-algebra in Sets. The functor $\underline{A}: \mathcal{C}(A) \rightarrow$ Sets is given on objects by

$$
\underline{A}(C)=C,
$$

and on morphisms $D \subseteq C$ of $\mathcal{C}(A)$ as the inclusion $\underline{A}(D) \hookrightarrow \underline{A}(C)$.

Note that the functor $\underline{A}: \mathcal{C}(A) \rightarrow$ Sets factors through CStar or cCstar via the forgetful embedding of CStar or cCstar in the ambient topos Sets.

\footnotetext{
${ }^{9}$ We will not worry about the fact that Topos, like Poset and CStar, is a large category; when pressed one can limit these categories to a chosen universe to make them small.
} 
Theorem 5. $\underline{A}$ is a commutative $C^{*}$-algebra in $\mathcal{T}(A)$ under the operations inherited from A. More precisely, $\underline{A}$ is a vector space over the internal complex numbers $\operatorname{Pt}(\underline{\mathbb{C}})$ (given simply by the constant functor $\operatorname{Pt}(\mathbb{C}): C \mapsto \mathbb{C}$ ) by

$$
\begin{array}{rlrl}
0 & : \underline{1} \rightarrow \underline{A} & 0_{C}(*) & =0, \\
+ & : \underline{A} \times \underline{A} \rightarrow \underline{A} & a+_{C} b & =a+b, \\
\cdot & : \operatorname{Pt}(\underline{\mathbb{C}}) \times \underline{A} \rightarrow \underline{A} & z \cdot{ }_{C} a & =z \cdot a,
\end{array}
$$

and an involutive algebra through

$$
\begin{array}{rlrl}
\cdot: & \underline{A} \times \underline{A} \rightarrow \underline{A} & a \cdot C b & =a \cdot b, \\
(-)^{*}: \underline{A} \rightarrow \underline{A} & \left(a^{*}\right)_{C}=a^{*} .
\end{array}
$$

The norm relation is given by

$$
N: \underline{A} \times \underline{\mathbb{Q}^{+}} \rightarrow \underline{\Omega} \quad N_{C}(a, q) \text { iff }\|a\|<q .
$$

Proof. One easily checks that the arrows are natural transformations (and hence morphisms in $\mathcal{T}(A)$ ) and that this structure satisfies the requirements for $\underline{A}$ to be a presemi-C*-algebra in $\mathcal{T}(A)$. Since each $\underline{A}(C)$ is a commutative $\mathrm{C}^{*}$-algebra in the ambient topos, $\underline{A}$ is commutative as well. (Alternatively, since the definition of a commutative pre-semi-C*-algebra consists only of geometrically definable objects $\left(\right.$ e.g. $\left.\mathbb{C}_{\mathbb{Q}}\right)$ and geometric formulae (see Appendix A and Sect. 2), it follows from Lemma 21 that $\underline{A}$ is a commutative pre-semi- $C^{*}$-algebra in $\mathcal{T}(A)$, because every $\underline{A}(C)$ is a commutative $\mathrm{C}^{*}$-algebra in the ambient topos.)

In fact, $\underline{A}$ is a pre-C*-algebra, i.e. internally the semi-norm is a norm: if for all $q>0$ we have $(a, q) \in N$, then $a=0$. To prove this, we need to show that $C \Vdash$ $\forall_{a \in \underline{A}_{\mathrm{sa}}} \forall_{q \in \mathbb{Q}^{+}} .(a, q) \in N \rightarrow a=0$, where we are using the internal language of $\mathcal{T}(A)$. In other words:

$$
\text { for all } C^{\prime} \supseteq C \text { and } a \in C^{\prime}, \text { if } C^{\prime} \Vdash \forall_{q \in \mathbb{Q}^{+}} .(a, q) \in N \text {, then } C^{\prime} \Vdash a=0 \text {, }
$$

i.e. for all $C^{\prime} \supseteq C$ and $a \in C^{\prime}$, if for all $C^{\prime \prime} \supseteq C^{\prime}$ and $q \in \mathbb{Q}^{+}$

$$
\text { we have } C^{\prime \prime} \Vdash(a, q) \in N \text {, then } C^{\prime} \Vdash a=0 \text {, }
$$

i.e. for all $C^{\prime} \supseteq C$ and $a \in C^{\prime}$, if $\|a\|=0$, then $a=0$.

But this holds, since every $C^{\prime}$ is a $C^{*}$-algebra.

Finally, $\underline{A}$ is in fact a $C^{*}$-algebra, i.e. internally we have Cauchy completeness. By the axiom of dependent choice (which holds because $\mathcal{T}(A)$ is a functor topos whose codomain validates dependent choice [41]) it suffices to prove that every regular Cauchy sequence (i.e. a sequence $\left(x_{n}\right)$ such that $\left\|x_{n}-x_{m}\right\| \leqslant 2^{-n}+2^{-m}$ for all $n, m$ ) converges. Thus we need to prove

$$
\begin{gathered}
C \Vdash \forall_{n, m} \cdot\left\|x_{n}-x_{m}\right\| \leqslant 2^{-n}+2^{-m} \rightarrow \exists_{x \in A} \cdot \forall_{n} \cdot\left\|x-x_{n}\right\| \leqslant 2^{-n}, \\
\text { i.e. for all } C^{\prime} \supseteq C, \text { if } C^{\prime} \Vdash\left(\forall_{n, m} \cdot\left\|x_{n}-x_{m}\right\| \leqslant 2^{-n}+2^{-m}\right), \\
\text { then } C^{\prime} \Vdash \exists_{x \in A} \cdot \forall_{n} \cdot\left\|x-x_{n}\right\| \leqslant 2^{-n},
\end{gathered}
$$

i.e. for all $C^{\prime} \supseteq C$, if $C^{\prime} \Vdash$ “ $x$ is regular", then $C^{\prime} \Vdash \exists_{x \in A} \cdot \forall_{n} \cdot\left\|x-x_{n}\right\| \leqslant 2^{-n}$.

Once again, this holds because every $C^{\prime}$ is a $C^{*}$-algebra. 
The functor $\underline{A}$ is our internal $C^{*}$-algebra. By changing the universe of discourse from the ambient topos Sets to $\mathcal{T}(A)$, the (generally) noncommutative $\mathrm{C}^{*}$-algebra $A$ has become a commutative $\mathrm{C}^{*}$-algebra $\underline{A}$. Multiplication of two non-commuting operators is no longer defined, since they live in different commutative subalgebras. ${ }^{10}$

3.3. The Kochen-Specker theorem. Combining the material in Sects. 2 and 3 so far, we obtain a mapping $A \mapsto \underline{\Sigma}(\underline{A})$, which associates a certain internal locale to a (generally) noncommutative $C^{*}$-algebra. As argued in the Introduction, $\underline{\Sigma}(\underline{A})$ describes the quantum logic of the physical system whose algebra of observables is $A$.

An important property of the internal spectrum $\Sigma$ is that it may typically be highly non-spatial from an external point of view. First, recall (see Subsect. 1.4) that a point of a locale $X$ in a topos $\mathcal{T}$ is a frame map $\mathcal{O}(X) \rightarrow \Omega$, where $\Omega$ is the subobject classifier in $\mathcal{T}$.

Theorem 6. Let $H$ be a Hilbert space with $\operatorname{dim}(H)>2$ and let $A$ be the $C^{*}$-algebra of bounded operators on $H$. Then the locale $\underline{\Sigma}(\underline{A})$ has no points.

Proof. We reason internally. A point $\rho: \underline{*} \rightarrow \underline{\Sigma}$ of the locale $\underline{\Sigma}$ (see Subsect. 1.5) may be combined with $a \in \underline{A}_{\text {sa }}$ with Gelfand transform $\hat{a}: \underline{\Sigma} \rightarrow \underline{\mathbb{R}}$ (see (25)), so as to produce a point $\hat{a} \circ \underline{\rho}: \underline{*} \rightarrow \underline{\mathbb{R}}$ of the locale $\underline{\mathbb{R}}$. This yields a map $\underline{V}_{\rho}: \underline{A}_{\mathrm{sa}} \rightarrow \operatorname{Pt}(\underline{\mathbb{R}})$, which can be shown to be an internal multiplicative functional; see $[6,7,27] .{ }^{11}$ Being an arrow in $\mathcal{T}(A)$, the map $\underline{V}_{\rho}$ is a natural transformation, with components $\underline{V}_{\rho}(C): \underline{A}_{\mathrm{sa}}(C) \rightarrow \operatorname{Pt}(\underline{\mathbb{R}})(C)$; by Definition 4 and 36, this is just $\underline{V}_{\rho}(C): C_{\text {sa }} \rightarrow \mathbb{R}$. Hence one has a multiplicative functional $\underline{V}_{\rho}(C)$ for each $C \in \mathcal{C}(\bar{A})$ in the usual sense, with the property (which follows from naturality) that if $C \subseteq D$, then the restriction of $\underline{V}_{\rho}(D)$ to $C_{\text {sa }}$ coincides with $\underline{V}_{\rho}(C)$. But this is precisely a valuation ${ }^{12}$ on $B(H)$, whose nonexistence was proved by Kochen and Specker [56].

This is a localic reformulation of the original topos-theoretic version of the KochenSpecker theorem due to Butterfield and Isham [20]. As in their work, the proof relies on the original version, but in being a statement about the nonexistence of models of a certain theory, our reformulation has a logical thrust that both the original version by Kochen and Specker and the reformulation by Butterfield and Isham lack.

The theorem certainly holds for more general $\mathrm{C}^{*}$-algebras than just the collection of all bounded operators on a Hilbert space; see [32 and 47] for results on von Neumann algebras. For $\mathrm{C}^{*}$-algebras, one has the result that a simple infinite unital $\mathrm{C}^{*}$-algebra does not admit a dispersion-free quasi-state [45]. Evidently, Theorem 6 holds for such extensions as well.

One way of looking at such results is to see them as illustrations of the failure of the Krein-Milman theorem in a constructive context [68]. Indeed, recall that the classical Krein-Milman theorem states that a compact convex set is the closed convex hull of its extreme points. The state space of $\underline{A}$ is still a compact convex set in an appropriate

10 Kochen and Specker refer to such a structure as a partial algebra [56] and stress its relevance for the foundations of quantum theory; in a partial algebra both addition and multiplication need only be defined for commuting operators.

11 This map may explicitly be given in the internal language of $\mathcal{T}(A)$, by noting that for each $a \in \underline{A}_{\mathrm{sa}}$ the expression $\tilde{\rho}(a)=\left(L_{\rho, a}, U_{\rho, a}\right)=\left(\left\{r \in \mathbb{Q} \mid \rho \models \mathrm{D}_{a-r}\right\},\left\{s \in \mathbb{Q} \mid \rho \models \mathrm{D}_{s-a}\right\}\right)$ is a Dedekind cut in $\mathcal{T}(A)$.

12 This terminology is to be distinguished from the one used in Subsect. 4.2 below. The naturality property just mentioned is often called noncontextuality in the philosophy of physics literature. 
localic sense (see Sect. 4), and the pure states on $\underline{A}$ would be its extreme boundary. These points, however, fail to exist, as we have just seen.

\section{4. (Quasi-)States as Integrals}

This section about states, and the next one about observables, are both concerned with connections between the two levels we have developed (see Fig. 1):

1. the ambient topos Sets, containing the $\mathrm{C}^{*}$-algebra $A$;

2. the associated topos $\mathcal{T}(A)$, containing the internal commutative $\mathrm{C}^{*}$-algebra $\underline{A}$ and its spectrum $\underline{\Sigma}$.

The main result of this section is Theorem 14, which gives an isomorphism between quasi-states on $A$ at level 1 and, at level 2, either probability integrals on $\underline{A}_{\text {sa }}$, or, equivalently, probability valuations on the Gelfand spectrum $\underline{\Sigma}$. Subsequently, we show that probability valuations define subobjects of $\underline{\Sigma}$, as in classical physics.

All this requires some preparation, firstly in the theory of quasi-states on $\mathrm{C} *$-algebras (Subsect. 4.1) and secondly in abstract constructive integration theory (Subsect. 4.2).

4.1. States and quasi-states. A linear functional $\rho: A \rightarrow \mathbb{C}$ on a $\mathrm{C}^{*}$-algebra $A$ is called positive when $\rho\left(a^{*} a\right) \geq 0$ for all $a \in A$. It is a state when it is positive and satisfies $\rho(1)=1$. A state $\rho$ is pure when $\rho=t \sigma+(1-t) \omega$ for some $t \in(0,1)$ and some states $\sigma$ and $\omega$ implies $\omega=\sigma$. Otherwise, it is called mixed. For example, if $A \subseteq B(H)$ for some Hilbert space $H$ (which we may always assume by the Gelfand-Naimark Theorem), then each unit vector $\Psi \in H$ defines a state $\psi$ on $A$ by $\psi(a)=(\Psi, a \Psi)$. If $A=B(H)$, such states are pure. (If $H$ is infinite-dimensional, not all pure states arise in this way, though.) Mixed states $\rho$ on $B(H)$ arise from countable sequences $\left(p_{i}\right), 0 \leqslant p_{i} \leqslant 1, \sum_{i} p_{i}=1$, coupled with an orthonormal family of vectors $\left(\Psi_{i}\right)$, through $\rho(a)=\sum_{i} p_{i} \psi_{i}(a)$. (By the spectral theorem, one may equivalently say that such states are given by positive operators $\hat{\rho}$ on $H$ with unit trace, through $\rho(a)=\operatorname{Tr}(\hat{\rho} a)$.) A state $\rho: A \rightarrow \mathbb{C}$ is called faithful when $\rho\left(a^{*} a\right)=0$ implies $a=0$. For example, if, in the situation just described, the $\Psi_{i}$ comprise an orthonormal basis of $H$ and each $p_{i}>0$, then the associated state $\rho$ is faithful. The states of a $C^{*}$-algebra form a compact convex set, the extremal points of which are by definition the pure states. States are automatically hermitian, in the sense that $\rho\left(a^{*}\right)=\overline{\rho(a)}$, or equivalently, $\rho(a) \in \mathbb{R}$ for self-adjoint $a$.

In algebraic quantum physics, mathematical states as defined above are often used to model the physical states of the quantum system. However, when taking Bohr's doctrine of classical concepts seriously, one should take into account that two observables can only be added in a physically meaningful way when they are jointly measurable, i.e. when the corresponding operators commute. Thus one may relax the definition of a quantum state, which ought to be linear only on commutative parts. This leads to the notion of a quasi-state [1]:13

13 Axiom VII of Mackey's foundation of quantum mechanics [64] states that a measure on the projections of a von Neumann algebra extends to a state on the von Neumann algebra. Mackey stresses that, in contrast to his other axioms, Axiom VII does not have a physical justification. One can prove that a measure extends to a quasi-state, so one is led to ask whether every quasi-state is a state. This is not the case when the von Neumann algebra has a summand of type $I_{2}$, but it holds for all other von Neumann algebras [15]. For $C^{*}$-algebras the question is more difficult. The main result seems to be the following [16]. Consider a $\mathrm{C}^{*}$-algebra with no quotient isomorphic to $M_{2}(\mathbb{C})$ and let $\rho$ be a quasi-linear functional. Then $\rho$ is linear iff $\rho$ restricted to the unit ball is uniformly weakly continuous. 
Definition 7. A quasi-linear functional on a $C^{*}$-algebra $A$ is a map $\rho: A \rightarrow \mathbb{C}$ that is linear on all commutative subalgebras and satisfies $\rho(a+i b)=\rho(a)+i \rho(b)$ for all self-adjoint $a, b \in A$ (possibly non-commuting). It is called positive when $\rho\left(a^{*} a\right) \geq 0$ for all $a \in A$. When $A$ is unital, a positive quasi-linear functional is called a quasi-state when $\rho(1)=1$.

This kind of quasi-linearity also determines when some property $P$ of the $\mathrm{C}^{*}$-algebra $A$ descends to a corresponding property $\underline{P}$ of the internal $\mathrm{C}^{*}$-algebra $\underline{A}$, as the following lemma shows. To be precise, for $P \subseteq A$, define a subfunctor of $\underline{A}$ by $\underline{P}(C)=P \cap C$. Let us call a property $P \subseteq A$ quasi-linear when $a \in P$ and $b \in P$ imply $\mu a+i \lambda b \in P$ for all $\mu, \lambda \in \mathbb{R}$ and $a, b \in A_{\text {sa }}$.

Lemma 8. Let $A$ be a $C^{*}$-algebra, and let $P \subseteq A$ be a quasi-linear property. Then $P=A$ if and only if $\underline{P}=\underline{A}$.

Proof. One implication is trivial; for the other, suppose that $\underline{P}=\underline{A}$. For $a \in A$, denote by $C^{*}(a)$ the sub-C*-algebra generated by $a$. When $a$ is self-adjoint, $C^{*}(a)$ is commutative. So $A_{\mathrm{sa}} \subseteq P$, whence by quasi-linearity of $P$ and the unique decomposition of elements in a real and imaginary part, we have $A \subseteq P$.

4.2. Algebraic integration theory. The well-known correspondence between states on commutative $\mathrm{C}^{*}$-algebras $A$ and probability measures on the underlying Gelfand spectrum $\Sigma$ is an immediate consequence of the Gelfand isomorphism $A \cong C(\Sigma, \mathbb{C})$ and the Riesz-Markov representation theorem in measure theory. In the present topos-theoretical setting, it turns out to be more natural to work with integrals and valuations rather than measures. Recall the a priori difference between these three concepts:

- measures are defined on Borel subsets of some space $X$;

- valuations are defined only on the open subsets of $X$;

- integrals are positive linear functionals on the (ordered) vector space $C_{c}(X, \mathbb{R})$.

Classically, if $X$ is locally compact Hausdorff and the measures in question are suitably regular, there are isomorphisms between these notions. From a constructive point of view, however, there is a subtle difference between valuations and integrals. ${ }^{14}$ In any case, the fundamental role locales play in this paper as the Gelfand spectra of the internal $\mathrm{C}^{*}$-algebras $\underline{A}$ makes it quite natural to assign probabilities to opens (rather than Borel subsets) of the spectrum.

The following string of definitions gives an abstract (and constructive) version of integration theory based on ordered vector spaces, abstracting from the Riemann, Lebesgue and Daniell integrals [30,31,75]. Several axiomatizations are possible, of which the one in terms of so-called f-algebras is the most convenient for our purposes.

14 The integral $I(f)$ of a function $f \in C(X)$ is a Dedekind real, so that it can be approximated by rationals. This may not be the case for the valuation $\mu(U)$ of an open $U$, as the 'kissing' property (if $r<s$ then $\mu(U)<s$ or $r<\mu(U))$ may fail. Accordingly, $\mu(U)$ is only a lower real, and can be thought of as a predicate $r<\mu(U)$ on the rationals. This predicate is downward closed: if $r<\mu(U)$ and $s \leqslant r$, then $s<\mu(U)$. But in general, given $\varepsilon>0$ one cannot approximate $\mu(U)$ up to $\varepsilon$ with rationals. Given an integral $I$, we can define a corresponding valuation $\mu_{I}(U)$ by taking the sup of $I(f)$ over all $0 \leqslant f \leqslant 1$ with support in $U$. It is remarkable that for any valuation $\mu$ one can conversely find a (unique) integral $I$ such that $\mu=\mu_{I}$. So despite the fact that one may not be able to compute $\mu(U)$, it is still possible to compute $\int f d \mu$ as a Dedekind real, which a priori is only a lower real. 
Definition 9. A Riesz space or vector lattice is a partially ordered vector space $(R, \leqslant)$ over $\mathbb{R}$ (i.e. a real vector space $R$ with partial ordering $\leqslant$ such that $f \leqslant g$ implies $f+h \leqslant g+h$ for all $h$ and $f \geq 0$ implies $r f \geq 0$ for all $r \in \mathbb{R}^{+}$) that is a distributive lattice with respect to its partial order [62, Definition 11.1].

Anf-algebra is a commutative, unital, real algebra $R$ whose underlying vector space is a Riesz space in which $f, g \geq 0$ implies $f g \geq 0$, and $f \wedge g=0$ implies $h f \wedge g=0$ for all $h \geq 0$. Moreover, the multiplicative unit 1 has to be strong in the sense that for each $f \in R$ there exists a natural number $n$ such that $-n 1 \leqslant f \leqslant n 1$ [82, Def. 140.8].

Note that although f-algebras are $a$ priori defined over the real numbers, they can $a$ fortiori be defined over $\mathbb{Q}$ as well.

The self-adjoint part of any commutative $\mathrm{C}^{*}$-algebra $A$ is an example of an f-algebra by defining $a \leqslant b$ in the usual way (i.e. iff $\exists_{c \in A}\left[b-a=c^{*} c\right]$ ); one has $f \vee g=$ $\max \{f, g\}$ and $f \wedge g=\min \{f, g\}$. Conversely, by the Stone-Yosida representation theorem every f-algebra can be densely embedded in a space of real continuous functions on a compact space.

Definition 10. An integral on an ordered vector space $R$ is a linear functional $I: R \rightarrow$ $\mathbb{R}$ that is positive, i.e. if $f \geq 0$ then also $I(f) \geq 0$. If $R$ has a strong unit 1 (e.g., the multiplicative unit in the case of f-algebras), then an integral I satisfying $I(1)=1$ is called a probability integral. An integral is faithful when its kernel is $\{0\}$, i.e., when $I(f)=0$ and $f \geq 0$ imply $f=0$.

Except in the degenerate case $I(1)=0$, any integral can obviously be normalised to a probability integral. The prime example of an integral is the Riemann or Lebesgue integral on the ordered vector space $C[0,1]$. More generally, any positive linear functional on a commutative $C^{*}$-algebra provides an example, states yielding probability integrals.

We wish to use a certain generalization of the Riesz-Markov theorem that can be proved constructively [30] and hence can be used within our topos $\mathcal{T}(A)$. This requires a localic reformulation of Definition 9 , as well as a similar approach to valuations.

Let $R$ be an $\mathrm{f}$-algebra (in Sets, for the moment). In defining the following frame it is technically convenient to define $R$ as a vector space over $\mathbb{Q}$. Define Integral $(R)$ as the distributive lattice freely generated by $\mathrm{P}_{f}, f \in R$, subject to the relations

$$
\begin{aligned}
\mathrm{P}_{1} & =\top, \\
\mathrm{P}_{f} \wedge \mathrm{P}_{-f} & =\perp, \\
\mathrm{P}_{f+g} & \left.\leqslant \mathrm{P}_{f} \vee \mathrm{P}_{g}, \quad \text { (for } f \leqslant 0\right) . \\
\mathrm{P}_{f} & =\perp
\end{aligned}
$$

This lattice generates a frame $\mathcal{O}(\mathcal{I}(R))$ by adding the regularity condition

$$
\mathrm{P}(f)=\bigvee_{\mathbb{Q} \ni q>0} \mathrm{P}(f-q)
$$

to the relations above, just like (23) in the case of the spectrum. It can be shown (cf. (17)) that

$$
\mathrm{P}_{f}=\{\rho: R \rightarrow \mathbb{R} \mid \rho(f)>0\},
$$

where each $\rho$ is understood to be a positive linear functional. Models of this theory, i.e. points of the associated locale, precisely correspond to probability integrals on $R$; if $I$ 
is such an integral, the associated model $m_{I}$ is given by $m_{I}\left(\mathrm{P}_{f}\right)=1$ iff $I(f)>0$. Conversely, a model $m$ defines an integral $I_{m}$ by (compare with the proof of Theorem 6 )

$$
I_{m}(f):=\left(\left\{p \mid m \models \mathrm{P}_{f-p}\right\},\left\{q \mid m \models \mathrm{P}_{q-f}\right\}\right),
$$

where the right-hand side is seen to be a Dedekind real from the relations on $\mathrm{P}_{\bullet}$. All this may be internalized to any topos, where, of course, there is no a priori guarantee that points of the locale with frame $\mathcal{O}(\mathcal{I}(R))$ exist (and hence that expressions like (41) make good sense).

The final ingredient of the constructive Riesz-Markov theorem is the definition of a locale of valuations. These were studied in [46 and 81].

Definition 11. A probability valuation on a locale $X$ is a monotone map $\mu: \mathcal{O}(X) \rightarrow$ $[0,1]_{l}$ that satisfies the usual additivity and regularity conditions for measures, i.e. $\mu(U)+\mu(V)=\mu(U \wedge V)+\mu(U \vee V)$ and $\mu\left(\bigvee_{\lambda} U_{\lambda}\right)=\bigvee_{\lambda} \mu\left(U_{\lambda}\right)$ for any directed family. (Here, $[0,1]_{l}$ is the collection of lower reals between 0 and 1.)

Like integrals, probability valuations on $X$ organize themselves in a locale $\mathcal{V}(X)$.

The generalized Riesz-Markov Theorem, then, is as follows.

Theorem 12 [30]. Let $R$ be an f-algebra and let $\Sigma$ be its spectrum. ${ }^{15}$ Then the locales $\mathcal{I}(R)$ and $\mathcal{V}(\Sigma)$ are isomorphic. To obtain an integral from a valuation we define:

$$
I_{\mu} f:=\sup _{\left(s_{i}\right)} \sum s_{i} \mu\left(s_{i}<f<s_{i+1}\right), \inf _{\left(s_{i}\right)} \sum s_{i+1}\left(1-\mu\left(s_{i}>f\right)-\mu\left(f>s_{i+1}\right)\right),
$$

where $(s<f)$ is a notation for $D_{f-s}$ and $(s<f<t)$ denotes $D_{f-s} \wedge D_{t-f}$ and $s_{i}$ is a partition of $[a, b]$ such that $a \leqslant f \leqslant b$. Conversely, to obtain a valuation from an integral I we define:

$$
\mu_{I}\left(D_{a}\right):=\sup \left\{I\left(n a^{+} \wedge 1\right) \mid n \in \mathbb{N}\right\}
$$

Note that both locales in question are compact regular [30]. Logically speaking, the theorem follows from the existence of a bi-interpretation between the geometric theories Integral $(R)$ and Valuation $(\Sigma)$ (i.e. there are interpretation maps in two directions which are each other's inverses) and the equivalence of the category of propositional geometric theories with interpretations to the category of frames.

4.3. From states on A to subobjects of $\mathcal{O}(\underline{\Sigma})$. We return to our main topic. Since everything in this section so far may be interpreted in the internal language of a topos and the proof of Theorem 12 is constructive, we have:

Corollary 13. Let $A$ be a $C^{*}$-algebra with Bohrification $\underline{A}$ and associated Gelfand spectrum $\underline{\Sigma}$ in the topos $\mathcal{T}(A)$. Then the locale $\mathcal{I}(\underline{A})$ of probability integrals on $\underline{A}_{\mathrm{sa}}$ is homeomorphic to the locale $\mathcal{V}(\underline{\Sigma})$ of probability valuations on $\underline{\Sigma}$.

As announced at the beginning of this section, the next theorem crosses two levels of Fig. 1.

15 See [27] for the notion of the spectrum of an f-algebra, which is described exactly as in Subsect. 2.2. If the $\mathrm{f}$-algebra is the self-adjoint part of a commutative $\mathrm{C}^{*}$-algebra, then its spectrum as an f-algebra coincides with its spectrum as a $\mathrm{C}^{*}$-algebra. 
Theorem 14. There is a bijective correspondence between quasi-states on A and either probability integrals on $\underline{A}_{\mathrm{sa}}$, or, equivalently, probability valuations on its Gelfand spectrum $\Sigma$.

This theorem may actually be extended to a correspondence between (faithful) positive quasi-linear functionals on $A$ and (faithful) integrals on $\underline{A}$, etc.

Proof. Every positive quasi-linear functional $\rho$ gives a natural transformation $I_{\rho}$ : $\underline{A}_{\mathrm{sa}} \rightarrow \underline{\mathbb{R}}$ if we define its components $\left(I_{\rho}\right)_{C}: C_{\mathrm{sa}} \rightarrow \mathbb{R}$ to be $\rho_{\mid C_{\mathrm{sa}}}$ (i.e. the restriction of $\rho$ to $\left.C_{\mathrm{sa}} \subseteq A_{\mathrm{sa}}\right)$.

Conversely, let $I: \underline{A}_{\mathrm{sa}} \rightarrow \underline{\mathbb{R}}$ be an integral. Define $\rho: A_{\mathrm{sa}} \rightarrow \mathbb{R}$ by

$$
\rho(a)=I_{C^{*}(a)}(a)
$$

For commuting $a, b \in A_{\text {sa }}$,

$$
\begin{aligned}
\rho(a+b) & =I_{C^{*}(a+b)}(a+b) \\
& =I_{C^{*}(a, b)}(a+b) \\
& =I_{C^{*}(a, b)}(a)+I_{C^{*}(a, b)}(b) \\
& =I_{C^{*}(a)}(a)+I_{C^{*}(b)}(b) \\
& =\rho(a)+\rho(b),
\end{aligned}
$$

because $I$ is a natural transformation, $C^{*}(a) \cup C^{*}(b) \subseteq C^{*}(a, b) \supseteq C^{*}(a+b)$, and $I$ is locally linear. Moreover, $\rho$ is positive because $I$ is locally positive (see Lemma 8). Hence we have defined $\rho$ on $A_{\mathrm{sa}}$ and may extend it to $A$ by complex linearity. It is clear that the two maps $I \mapsto \rho$ and $\rho \mapsto I$ are inverses of each other and that if $I$ is a probability integral, then $\rho$ is a quasi-state, and vice versa.

In the Introduction, we have seen that in the classical case a (pure) state $\rho$ defines a subobject $[\rho]$ of the frame of opens of the classical phase space; see (3). As we shall now show, this remains true, mutatis mutandis, in the quantum case. The main technical difficulty is to adapt the condition $\delta_{\rho}(V)=1$ in (3).

Theorem 14 yields a bijective correspondence between quasi-states $\rho$ on $A$ and probability valuations $\mu_{\rho}$ on $\underline{\Sigma}$. Fix a state, or quasi-state, $\rho$ on $A$. The logical formula $\mu_{\rho}(-)=1$ (of the Mitchell-Bénabou language of $\mathcal{T}(A)$ ) is a predicate on $\mathcal{O}(\underline{\Sigma})$ and hence defines a subobject $[\rho]$ of $\mathcal{O}(\underline{\Sigma})$ with characteristic arrow $\chi_{[\rho]}: \mathcal{O}(\underline{\Sigma}) \rightarrow \underline{\Omega}$. This arrow is just the interpretation of $\mu_{\rho}(-)=1$, i.e.

$$
\chi_{[\rho]}=\llbracket \mu_{\rho}(-)=1 \rrbracket .
$$

Compare with (3); beyond mimicking the notation, we see that we have been able to transfer the classical description of states to the quantum situation in every respect.

\section{Observables and Propositions}

In this section and the next we give the details of Steps 2 to 5 of our five-step program for spatial quantum logic, $c f$. Subsects. 1.2 and 1.6. We start with the locale map $\delta(a): \underline{\Sigma} \rightarrow \underline{\mathbb{R}}$, then turn to the description of elementary propositions $a \in \Delta$ as opens in the spectrum $\underline{\Sigma}$, and finally consider the pairing of states and propositions to arrive at a suitable notion of (multi-valued) truth in quantum theory. 
5.1. Interval domain. For a commutative unital $\mathrm{C}^{*}$-algebra $A$ with Gelfand spectrum $\Sigma$ in Sets, the Gelfand transform of $a \in A_{\mathrm{sa}}$ is a continuous function $\hat{a}: \Sigma \rightarrow \mathbb{R}$. Equivalently, it is a locale map (25). As we have seen in Subsect. 2.3, mutatis mutandis the description (25) still applies when $A$ is a commutative unital $\mathrm{C}^{*}$-algebra $A$ with Gelfand spectrum $\Sigma$ in a topos $\mathcal{T}$. In particular, one has the Gelfand transform

$$
\hat{a}: \underline{\Sigma} \rightarrow \underline{\mathbb{R}} \quad\left(a \in \underline{A}_{\mathrm{sa}}\right) .
$$

Our problem, however, is to express an element $a \in A_{\mathrm{sa}}$ of a noncommutative $C^{*}$-algebra $A$ in Sets in terms of some locale map $\delta(a)$ defined on the spectrum $\underline{\Sigma}$ of the Bohrification $\underline{A}$ of $A$ in $\mathcal{T}(A)$. As we shall see, this problem can be solved if we introduce some fuzziness, in that $\delta(a)$ no longer takes values in the internal Dedekind reals $\mathbb{R}$ in $\mathcal{T}(A)$, like $\hat{a}$, but in the so-called interval domain $\mathbb{I}$, internalized in $\mathcal{T}(A)$ as $\underline{\mathbb{R}}$. Thus, apart from (43) we are dealing with a second locale map

$$
\underline{\delta}(a): \underline{\Sigma} \rightarrow \underline{\mathbb{R}} \quad\left(a \in A_{\mathrm{sa}}\right) .
$$

In honour of Döring and Isham, we refer to $\underline{\delta}(a)$ as the Daseinisation of $a$ (although our map differs from theirs, $c f$. Appendix B).

We have already encountered Scott's interval domain $\mathbb{I R}$ in Subsect. 1.8 as the poset of compact intervals in $\mathbb{R}$, ordered by inverse inclusion. Like the Dedekind real numbers, the interval domain is easily internalized and hence definable in any topos. In fact, the construction of the Dedekind real numbers in Subsect. 2.3 only requires a single modification so as to obtain the interval domain: the corresponding frame $\mathcal{O}(\mathbb{I})$ is defined by the very same generators $(p, q)$ and relations as $\mathcal{O}(\mathbb{R})$, except that the fourth relation (i.e. $(p, q)=\left(p, q_{1}\right) \vee\left(p_{1}, q\right)$ if $\left.p \leqslant p_{1} \leqslant q_{1} \leqslant q\right)$ is dropped. The models of $\mathcal{O}(\mathbb{I} \mathbb{R})$ or points of the associated locale $\mathbb{I} \mathbb{R}$ again correspond to pairs $(L, U)$ given by (31) and (32), but this time such a pair may fail to define a Dedekind cut; axiomatically, only the 'kissing' requirement no longer holds. In any topos $\mathcal{T}$, we denote the locale defined by the geometric propositional theory given by the first three axioms in the list following (30) in Subsect. 2.3-interpreted in $\mathcal{T}$-by $\mathcal{O}(\mathbb{R})_{\mathcal{T}}$, with the usual special case $\underline{\mathbb{R}} \equiv \mathbb{R}_{\mathcal{T}(A)}$. Similarly, the subobject of $\mathcal{P}(\mathbb{Q}) \times \mathcal{P}(\mathbb{Q})$ consisting of models of $\mathcal{O}(\mathbb{I R})_{\mathcal{T}}$ is denoted by $\operatorname{Pt}(\mathbb{I R})_{\mathcal{T}}$, with $\operatorname{Pt}(\mathbb{R})_{\mathcal{T}(A)} \equiv \operatorname{Pt}(\underline{\mathbb{R}})$.

The examples in Subsect. 2.3 now read as follows:

1. In Sets (or, more generally, when classical logic applies in $\mathcal{T}$ ), a cut $(L, U)$ defines a compact interval [sup $L$, inf $U$ ] (where sup and inf are taken in $\mathbb{R}$ ), so that $\operatorname{Pt}(\mathbb{R}$ ) may be identified with the classical Scott interval domain $\mathbb{R}$. In that case, a generator $(p, q) \in \mathcal{O}(\mathbb{I})$ may be identified with the Scott open in $\mathbb{I}$ that contains all intervals $[a, b]$ such that $p<a \leqslant b<q$.

2. In a topos $\operatorname{Sh}(X)$ of sheaves one has

$$
\mathcal{O}(\mathbb{R})_{\operatorname{Sh}(X)}: U \mapsto \mathcal{O}(U \times \mathbb{I R}),
$$

but its points are not as easily described as (34); instead, one has

$$
\begin{aligned}
& \operatorname{Pt}(\mathbb{R}): U \mapsto\{(f, g)|f, g: U \rightarrow \mathbb{R}| f \leqslant g, \\
& \quad f \text { lower-semicont., } g \text { upper-semicont. }\} .
\end{aligned}
$$

This follows by carefully adapting the proof of [63, Theorem VI.8.2] for $\mathbb{R}$. 
3. In particular, for $\mathcal{T}(A)=\operatorname{Sets}^{\mathcal{C}(A)}$, one has

$$
\mathcal{O}(\underline{\mathbb{R}}): C \mapsto \mathcal{O}((\uparrow C) \times \mathbb{I R})
$$

which may be identified with the set of monotone functions from $\uparrow C$ to $\mathcal{O}(\mathbb{I} \mathbb{R}) .{ }^{16}$ The object $\mathrm{Pt}(\underline{\mathbb{R}})$ will not be used in this paper. ${ }^{17}$

5.2. Daseinisation. After this preparation, we turn to the Daseinisation (44), or rather to the corresponding frame map

$$
\underline{\delta}(a)^{-1}: \mathcal{O}(\underline{\mathbb{R}}) \rightarrow \mathcal{O}(\underline{\Sigma}) .
$$

A complete description of this map, based on the technique of generating (semi)lattices for frames, may be found in Appendix A.2. Here, we just look at the special case

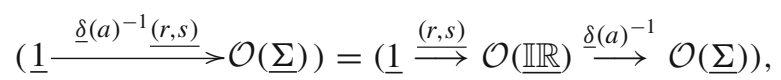

where the arrow $(r, s): \underline{1} \rightarrow \mathcal{O}(\underline{\mathbb{R}})$ maps into the monotone function with constant

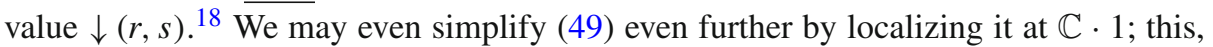
however, entails no loss of generality, for $\mathcal{O}(\underline{\Sigma})(\mathbb{C} \cdot 1)$ is the frame in Sets that (together with the frame map (88)) provides the external description of the internal locale $\underline{\Sigma}$ in $\mathcal{T}(A)$ (see Subsect. 1.7 and Appendix A.2).

The quantity $\underline{\delta}(a)^{-1}(r, s)(\mathbb{C} \cdot 1)$ is a global element $\underline{U}$ of $\mathcal{O}(\Sigma)(\mathbb{C} \cdot 1)$ as described by Theorem 29 in Appendix A. Briefly, this theorem states that $\mathcal{\mathcal { O } ( \Sigma )}(\mathbb{C} \cdot 1)$ may be seen as the set of all subfunctors $\underline{U}$ of the functor $C \mapsto L_{C}$ that satisfy a certain regularity condition, where $L_{C}$ is the distributive lattice freely generated by the formal symbols $\mathrm{D}_{c}$, $c \in C_{\mathrm{sa}}$ subject to the relations (18)-(22) (simply interpreted in Sets). ${ }^{19}$ Abbreviating

$$
\delta(a)^{-1}(r, s)=\underline{\delta}(a)^{-1} \underline{(r, s)}(\mathbb{C} \cdot 1),
$$

the ensuing element $\delta(a)^{-1}(r, s)$ of $\mathcal{O}(\underline{\Sigma})(\mathbb{C} \cdot 1)$ turns out to be the functor

$$
\delta(a)^{-1}(r, s): C \mapsto\left\{D_{f-r} \wedge D_{s-g} \mid f, g \in C_{\mathrm{sa}}, f \leqslant a \leqslant g\right\} .
$$

This follows from (106) and the definition of $\underline{\delta}(a)^{-1}$ in Appendix A.2, combined with the equality

$$
\begin{gathered}
\bigcup_{\{p<q \mid(p, q) \subseteq(r, s)\}}\left\{D_{f-p} \wedge D_{q-g} \mid f, g \in C_{\mathrm{sa}}, f \leqslant a \leqslant g\right\} \\
=\left\{D_{f-r} \wedge D_{s-g} \mid f, g \in C_{\mathrm{sa}}, f \leqslant a \leqslant g\right\} .
\end{gathered}
$$

\footnotetext{
16 The argument is the same as for $\mathbb{R}$, see footnote 7 .

17 For completeness, we mention that $\operatorname{Pt}(\underline{\mathbb{R}})(C)$ is the set of all pairs $(L, U)$, where $L$ and $U$ are subfunctors of the constant functor $\mathbb{Q}$, truncated to $\uparrow C \subset \mathcal{C}(A)$, such that for all $D \supseteq C,(L(D), U(D))$ is a pair of the form (31)-(32).

18 Here $(r, s)$ is seen as an element of the generating semilattice $\mathbb{Q} \times<\mathbb{Q}$, whereas $\downarrow(r, s)$ is its image in the frame $\mathcal{O}(\mathbb{I R})$ through the canonical map (67); see Appendix A.

19 See Appendix A.1 for a detailed description of the functor $C \mapsto L_{C}$.
} 
An alternative description of $\delta(a)^{-1}$ is as follows. For fixed $a \in A_{\mathrm{sa}}$, define functors $\underline{L_{a}} \in \mathcal{T}(A)$ and $\underline{U_{a}} \in \mathcal{T}(A)$ by

$$
\begin{aligned}
& \underline{L_{a}}(C)=\left\{f \in C_{\mathrm{sa}} \mid f \leqslant a\right\} ; \\
& \underline{U_{a}}(C)=\left\{g \in C_{\mathrm{sa}} \mid a \leqslant g\right\} .
\end{aligned}
$$

Each of these defines a subobject of $\underline{A}_{\mathrm{sa}}$. In fact, the pair $\left(L_{a}, U_{a}\right)$ is a directed subobject of $\underline{A}_{\mathrm{sa}} \times \underline{A}_{\mathrm{sa}}^{\mathrm{op}}$. Now take a generator $(r, s)$ of $\mathcal{O}(\mathbb{I R})$, and write $(r, s)=(-\infty, s) \wedge(r, \infty)$. The Gelfand transform (27) defines subobjects $\hat{\imath}\left(\underline{L_{a}},(r, \infty)\right)$ and $\hat{\imath}\left(\underline{U_{a}},(-\infty, s)\right)$ of $\mathcal{O}(\underline{\Sigma})$. We then put

$$
\delta(a)^{-1}:(r, s) \mapsto \bigvee \hat{\imath}\left(\underline{L_{a}},(r, \infty)\right) \wedge \hat{\imath}\left(\underline{U_{a}},(-\infty, s)\right)
$$

Using (28) and (29), this gives ${ }^{20}$

$$
\delta(a)^{-1}(r, s)=\bigvee_{f \in \underline{L_{a}}, g \in \underline{U_{a}}} \mathrm{D}_{f-r} \wedge \mathrm{D}_{s-g} .
$$

To illustrate what is going on, it is helpful to compute the right-hand side of (51) or (54) in Sets for $A=C=C(\Sigma, \mathbb{C})$. In that case the meaning of $\mathrm{D}_{a}$ is given by (17), so that with $\rho(f)=f(\rho)$ one finds $\mathrm{D}_{f-r}=\{\rho \in \Sigma \mid f(\rho)>r\}$ and $\mathrm{D}_{s-g}=\{\rho \in \Sigma \mid g(\rho)<s\}$. One then obtains (with $\wedge$ for 'and')

$$
\begin{aligned}
\delta(a)_{A}^{-1}(r, s) & =\bigcup_{f, g \in C_{\mathrm{sa}}, f \leqslant a \leqslant g}\{\rho \in \Sigma \mid f(\rho)>r \wedge g(\rho)<s\} \\
& =\left\{\rho \in \Sigma \mid \exists_{f \leqslant a}[f(\rho)>r \wedge f(\rho)<s] \wedge \exists_{g \geq a}[g(\rho)>r \wedge g(\rho)<s]\right\} \\
& =\{\rho \in \Sigma \mid r<a(\rho)<s\} \\
& =a^{-1}(r, s) .
\end{aligned}
$$

To close this subsection, we note the following:

Proposition 15. The map $\delta: A_{\mathrm{sa}} \rightarrow C(\underline{\Sigma}, \underline{\mathbb{R}})$ is injective, and $a \leqslant b$ iff $\delta(a) \leqslant \delta(b)$.

Proof. Suppose that $\delta(a)=\delta(b)$. Then for all $C \in \mathcal{C}(A)$, the sets $L_{a}(C)=\left\{f \in C_{\text {sa }} \mid\right.$ $f \leqslant a\}$ and $U_{a}(C)=\left\{g \in C_{\text {sa }} \mid a \leqslant g\right\}$ must coincide with $L_{b}(C)$ and $U_{b}(C)$, respectively. Imposing these equalities at $C=C^{*}(a)$ and at $C=C^{*}(b)$ yields $a=b$. The order in $A_{\text {sa }}$ is clearly preserved by $\delta$, whereas the converse implication can be shown by the same method as the first claim of the proposition.

${ }^{20}$ Using a generic point $\sigma$, we may even define

$$
\delta(a)(\sigma):=\left(\sup \sigma\left(\underline{L_{a}}\right), \inf \sigma\left(\underline{U_{a}}\right)\right)
$$

Analoguously, one can view $\delta(a)$ as an interpretation of the geometric theory $\Sigma$ in the geometric theory of the intervals, see [30]. 
5.3. Propositions. It immediately follows from the existence of the Daseinisation map (48) (see Subsect. 5.2 and Appendix A.2) that, as in the classical case, elementary propositions $a \in \Delta$ define opens in phase space. For an open in the 'quantum phase space' $\underline{\Sigma}$ is simply defined as a global element $\underline{1} \rightarrow \mathcal{O}(\underline{\Sigma})$ (cf. Subsect. 1.4)), so that given an observable $a \in A_{\mathrm{sa}}$ and a Scott open $\Delta \in \mathcal{O}(\underline{\mathbb{R}})$, we may combine the corresponding arrows $\underline{\delta}(a)^{-1}: \mathcal{O}(\underline{\mathbb{I R}}) \rightarrow \mathcal{O}(\underline{\Sigma})$ and $\underline{\Delta}: \underline{1} \rightarrow \mathcal{O}(\underline{\mathbb{I R}})$ into

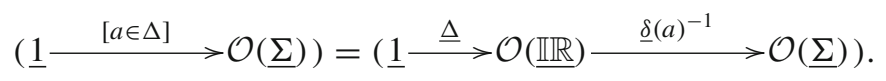

This generalises (49); in particular, $\underline{\Delta}: \underline{1} \rightarrow \mathcal{O}(\underline{\mathbb{I}})$ is defined at $C$ as the monotone function $\uparrow C \rightarrow \mathcal{O}(\mathbb{R})$ taking constant value $\Delta$. In other words,

$$
[a \in \Delta]=\underline{\delta}(a)^{-1} \circ \underline{\Delta} .
$$

\section{State-Proposition Pairing}

In Subsect. 4.3 we have shown how a state $\rho$ on $A$ gives rise to a subobject $[\rho]$ of $\mathcal{O}(\Sigma)$ defined by the predicate $\mu_{\rho}(-)=1$, and hence to an arrow $\mathcal{O}(\underline{\Sigma}) \stackrel{\chi_{[\rho]}}{\longrightarrow} \underline{\Omega}$ related to the predicate in question by (42).

Also, we have just seen the description (56) of propositions $a \in \Delta$ as opens in $\underline{\Sigma}$. Hence we can pair a physical state $\rho$ and a physical proposition $a \in \Delta$ by composition, to end up with a 'truth value' $\langle a \in \Delta, \rho\rangle$ in the subobject classifier $\underline{\Omega}$ of $\mathcal{T}(A)$. Explicitly, one has

$$
(\underline{1} \stackrel{\langle a \in \Delta, \rho\rangle}{\longrightarrow} \underline{\Omega})=\left(\underline{1} \stackrel{[a \in \Delta]}{\longrightarrow} \mathcal{O}(\underline{\Sigma}) \stackrel{\chi_{[\rho]}}{\longrightarrow} \underline{\Omega}\right),
$$

or

$$
\langle a \in \Delta, \rho\rangle=\chi_{[\rho]} \circ \underline{\delta}(a)^{-1} \circ \underline{\Delta} .
$$

In what follows, we need the basic definitions of Kripke-Joyal semantics. If $\varphi$ is some formula interpreted in a topos $\mathcal{T}$ as an arrow $\llbracket \varphi \rrbracket: F \rightarrow \Omega$, and $\alpha: B \rightarrow F$ is any arrow in $\mathcal{T}$ (defining a 'generalized element' of $F$ ), then the notation $B \Vdash \varphi(\alpha)$, or, less precisely, $B \Vdash \varphi$ (for ' $B$ forces $\varphi$ ') means that the composite arrow $B \stackrel{\alpha}{\rightarrow} F \stackrel{\llbracket \varphi \rrbracket}{\longrightarrow} \Omega$ factors through $\top: 1 \rightarrow \Omega$. In a functor topos Sets $^{\mathbf{C}}$, where $\mathbf{C}$ is some category, the notation $C \Vdash \varphi$ for some $C \in \mathbf{C}$ is shorthand for $y(C) \Vdash \varphi$, where $y(C): D \mapsto \operatorname{Hom}_{\mathbf{C}}(D, C)$ is the Yoneda functor. In our case $\mathcal{T}=\mathcal{T}(A)$, the interpretation $\llbracket \varphi \rrbracket$ is a natural transformation $\underline{F} \rightarrow \underline{\Omega}$, given by its components $\llbracket \varphi \rrbracket(C): \underline{F}(C) \rightarrow \underline{\Omega}(C)$, where $C \in \mathcal{C}(A)$. In that case the forcing condition $C \Vdash \varphi$ turns out to be equivalent to $\llbracket \varphi \rrbracket(C)(\underline{F}(C))=\top_{C}$, where $\top_{C}$ is the maximal upper set on $C$.

Using the Kripke-Joyal semantics of $\mathcal{T}(A)$, we now explicitly compute the stateproposition pairing in case that $\Delta=(r, s)$ is a rational interval. The computation is straightforward when using generating lattices (see Appendix A). From here on, $\rho$ is a fixed state on $A$ and we abbreviate $\mu_{\rho}$ by $\mu$. For $D \in \mathcal{C}(A)$,

$$
\begin{aligned}
& (\langle a \in(r, s), \rho\rangle)_{D}(*) \stackrel{(58)}{=} \quad\left(\chi_{[\rho]} \circ[a \in(r, s)]\right)_{D}(*) \\
& \stackrel{(57),(42)}{=} \llbracket \mu\left(\underline{\delta}(a)^{-1}(r, s)\right)=1 \rrbracket(D) \text {. }
\end{aligned}
$$


Being a global element $\underline{1} \rightarrow \underline{\Omega}$ of the subobject classifier $\underline{\Omega}$ of $\mathcal{T}(A)$, the right-hand side is an element of the set $\underline{\Omega(D)}$, and hence an upper set on $D$. With slight abuse of notation, we simply call the latter $\langle a \in(r, s), \rho\rangle(D)$. It follows that

$$
\langle a \in(r, s), \rho\rangle(D)=\left\{C \in \mathcal{C}(A) \mid C \supseteq D, C \Vdash \mu\left(\underline{\delta}(a)^{-1}(r, s)\right)=1\right\},
$$

where $\mu \circ \underline{\delta}(a)^{-1}(-)=1$ is the obvious predicate on $\mathcal{O}(\underline{\mathbb{I R}})$ defined by $\mu(-)=1$ on $\mathcal{O}(\underline{\Sigma})$ and the Daseinisation map (48). Since $\langle a \in(r, s), \rho\rangle(D)$ is the truncation to $\uparrow D$ of the corresponding upper set at $\mathbb{C} \cdot 1$, we may use (51) or (54), from which we see that the forcing condition $C \Vdash \mu\left(\underline{\delta}(a)^{-1}(r, s)\right)=1$ is equivalent to

$$
\mu_{C}\left(\bigvee_{f \leqslant a \leqslant g, f, g \in C_{\mathrm{sa}}} \mathrm{D}_{f-r} \wedge \mathrm{D}_{s-g}\right)=1 .
$$

Here $\mu_{C}$ is the valuation defined as $\mu_{\rho}$, but with $\rho$ restricted to $C$. Similarly, $\mathrm{D}_{f-r}$ refers to an open in the spectrum of $C$ ( $c f$. Theorem 20, according to which the $\mathrm{D}_{a}$ with $a \in C_{\mathrm{sa}}$ may be seen as generators of the spectrum of $C$ ). Since the measure of the intersection of two opens equals one if the measures of both opens do, this means (for $f, g \in C_{\mathrm{sa}}$ )

$$
\mu_{C}\left(\left(\bigvee_{f \leqslant a} \mathrm{D}_{f-r}\right) \wedge\left(\bigvee_{g \geq a} \mathrm{D}_{s-g}\right)\right)=1,
$$

which happens if and only if

$$
\mu_{C}\left(\bigvee_{f \leqslant a} \mathrm{D}_{f-r}\right)=1 \text { and } \mu_{C}\left(\bigvee_{g \geq a} \mathrm{D}_{s-g}\right)=1
$$

The left conjunct means

$$
\forall_{n \in \mathbb{N}} \exists_{f \in C_{\mathrm{sa}}, f \leqslant a}\left[\mu_{C}\left(\mathrm{D}_{f-r}\right)>1-\frac{1}{n}\right],
$$

since $\mathcal{T}(A)$ is a functor topos and hence the quantifiers above are interpreted locally. The construction of $\mu_{\rho}$ from $\rho$ (see Sect. 4) implies

$$
\mu_{C}\left(D_{h}\right)=\lim _{m \rightarrow \infty} \rho\left(\left(m h^{+}\right) \wedge 1\right),
$$

where the limit is a lower real. In other words, $\mu_{C}\left(D_{h}\right)>q$ iff there exists $m$ in $\mathbb{N}$ such that $\rho\left(\left(m h^{+}\right) \wedge 1\right)>q$. So $C \Vdash \mu_{\rho}\left(\bigvee_{f \leqslant a} \mathrm{D}_{f-r}\right)=1$ means that for each $n \in \mathbb{N}$ there exists $f \in C$ with $f \leqslant a$ and $\mu_{C}\left(\mathrm{D}_{f-r}\right)>1-\frac{1}{n}$.

Hence at the end of the day the state-proposition pairing $\langle a \in(r, s), \rho\rangle$ explicitly yields the upper set at $D$ given by

$$
\begin{aligned}
& \langle a \in(r, s), \rho\rangle(D) \\
& =\left\{C \in \mathcal{C}(A) \mid C \supseteq D, \mu_{C}\left(\bigvee_{f \leqslant a, f \in C_{\mathrm{sa}}} \mathrm{D}_{f-r}\right)=1 \text { and } \mu_{C}\left(\bigvee_{a \leqslant g, g \in C_{\mathrm{sa}}} \mathrm{D}_{s-g}\right)=1\right\} .
\end{aligned}
$$


This formula can be put in a slightly more palpable form when $A$ and each $C \in \mathcal{C}(A)$ are von Neumann algebras (in the ambient topos Sets). In that case, it can be shown [47] that the open $\mathrm{D}_{f-r}$ in the spectrum gives rise to a projection operator $\left[\mathrm{D}_{f-r}\right]$, to which we can directly apply the state $\rho$. Moreover, unlike for general $\mathrm{C}^{*}$-algebras, the supremum $P=\bigvee\left\{\left[\mathrm{D}_{f-r}\right] \mid f \leqslant a, f \in C_{\mathrm{sa}}\right\}$ exists. One then simply has $\mu_{C}(P)=1$ when $\rho(P)=1$. Similarly, the projection $Q=\bigvee\left\{\left[\mathrm{D}_{s-g}\right] \mid a \leqslant g, g \in C_{\text {sa }}\right\}$ exists and $\mu_{C}(Q)=1$ when $\rho(Q)=1$.

To close, we remark that one might consider a proposition $\mu_{\rho}(-)>p$, for some rational number $p$, instead of the proposition $\mu_{\rho}(-)=1$ as in this paper. This would simplify the computations above slightly. For instance, (61) would become

$$
\exists_{f \in C_{\mathrm{sa}}, f \leqslant a}\left[\mu_{C}\left(\mathrm{D}_{f-r}\right)>p\right] .
$$

This eliminates a universal quantification, but otherwise the computations would continue mutatis mutandis as before.

\section{A. Generating Lattices for Frames}

At various places in this article we refer to a presentation of a frame (or locale) by a generating lattice with a covering relation. This technique has been developed in the context of formal topology [71,72], and extends an analogous construction due to Johnstone [48]. Note that formal topology may be developed in the framework of constructive set theory [3], and hence may be internalized in topos theory.

Let $(L, \leqslant)$ be a meet semilattice (i.e. a poset in which any pair of elements has a meet $=$ g.l.b. $=$ infimum; in most of our applications $(L, \leqslant)$ is actually a distributive lattice).

Definition 16. A covering relation on $L$ is a relation $\triangleleft \subseteq L \times \mathcal{P}(L)$ - equivalently, a function $L \rightarrow \mathcal{P}(\mathcal{P}(L))$ - written $x \triangleleft U$ when $(x, U) \in \triangleleft$, such that:

1. If $x \in U$ then $x \triangleleft U$;

2. If $x \triangleleft U$ and $U \triangleleft V$ (i.e. $y \triangleleft V$ for all $y \in U$ ) then $x \triangleleft V$;

3. If $x \triangleleft U$ then $x \wedge y \triangleleft U$;

4. If $x \in U$ and $x \in V$, then $x \triangleleft U \wedge V$ (where $U \wedge V=\{x \wedge y \mid x \in U, y \in V\}$ ).

For example, if $(L, \leqslant)=(\mathcal{O}(X), \subseteq)$ one may take $x \triangleleft U$ iff $x \leqslant \bigvee U$, i.e. iff $U$ covers $x$.

Let $D L$ be the poset of all lower sets in $L$, ordered by inclusion; this is a frame [48, Sect. 1.2]. The structure $\triangleleft$ gives rise to a closure operation ${ }^{21} \mathcal{A}: D L \rightarrow D L$, given by

$$
\mathcal{A} U=\{x \in L \mid x \triangleleft U\},
$$

which has the following properties: $\downarrow U \subseteq \mathcal{A} U, U \subseteq \mathcal{A} V \Rightarrow \mathcal{A} U \subseteq \mathcal{A} V, \mathcal{A} U \cap \mathcal{A} V \subseteq$ $\mathcal{A}(\downarrow U \cap \downarrow V)$. The frame $\mathcal{F}(L, \triangleleft)$ generated by such a structure is then defined by

$$
\mathcal{F}(L, \triangleleft)=\{U \in D L \mid \mathcal{A} U=U\}=\{U \in \mathcal{P}(L) \mid x \triangleleft U \Rightarrow x \in U\} ;
$$

the second equality follows because firstly the property $\mathcal{A} U=U$ guarantees that $U \in D L$, and secondly one has $\mathcal{A} U=U$ iff $x \triangleleft U$ implies $x \in U$. An equivalent description of $\mathcal{F}(L, \triangleleft)$ is

$$
\mathcal{F}(L, \triangleleft) \cong \mathcal{P}(L) / \sim,
$$

21 As a map, $\mathcal{A}$ is also defined on $\mathcal{P}(L)$. Let $\chi \triangleleft: L \times \mathcal{P}(L) \rightarrow \Omega$ be the characteristic function of the subset $\triangleleft \subseteq L \times \mathcal{P}(L)$. Then $\mathcal{A}=\hat{\chi} \triangleleft$ is just the 'curry' or ' $\lambda$-conversion' of $\chi \triangleleft$. 
where $U \sim V$ iff $U \triangleleft V$ and $V \triangleleft U$. Indeed, the map $U \mapsto[U]$ from $\mathcal{F}(L, \triangleleft)$ (as defined in (64)) to $\mathcal{P}(L) / \sim$ is a frame map with inverse $[U] \mapsto \mathcal{A} U$; hence the idea behind the isomorphism (65) is that the map $\mathcal{A}$ picks a unique representative in the equivalence class $[U]$, namely $\mathcal{A} U$.

The frame $\mathcal{F}(L, \triangleleft)$ comes equipped with a canonical map

$$
\begin{aligned}
f: L & \rightarrow \mathcal{F}(L, \triangleleft) ; \\
x & \mapsto \mathcal{A}(\downarrow x),
\end{aligned}
$$

which satisfies $f(x) \leqslant \bigvee f(U)$ if $x \triangleleft U$. In fact, $f$ is universal with this property, in that any homomorphism $g: L \rightarrow \mathcal{G}$ of meet semilattices into a frame $\mathcal{G}$ such that $g(x) \leqslant \bigvee g(U)$ whenever $x \triangleleft U$ has a factorisation $g=\varphi \circ f$ for some unique frame map $\varphi: \mathcal{F}(L, C) \rightarrow \mathcal{G}$. This suggests that the point of the construction is that $\mathcal{F}(L, \triangleleft)$ is (isomorphic to) a frame defined by generators and relations, provided the covering relation is suitably defined in terms of the relations. More precisely [3, Thm. 12]:

Proposition 17. Suppose one has a frame $\mathcal{F}$ and a meet semilattice ${ }^{22} L$ with a map $f: L \rightarrow \mathcal{F}$ of meet semilattices that generates $\mathcal{F}$ in the sense that for each $U \in \mathcal{F}$ one has $U=\bigvee\{f(x) \mid x \in L, f(x) \leq U\}$. Define a cover relation $\triangleleft$ on $L$ by

$$
x \triangleleft U \text { iff } f(x) \leqslant \bigvee f(U) .
$$

Then one has a frame isomorphism $\mathcal{F} \cong \mathcal{F}(L, \triangleleft)$.

We now turn to maps between frames.

Definition 18. Let $(L, \triangleleft)$ and $(M, \triangleleft)$ be meet semilattices with covering relation as above, and let $f^{*}: L \rightarrow \mathcal{P}(M)$ be such that:

1. $f^{*}(L)=M ;^{23}$

2. $f^{*}(x) \wedge f^{*}(y) \triangleleft f^{*}(x \wedge y)$;

3. $x \triangleleft U \Rightarrow f^{*}(x) \triangleleft f^{*}(U)$ (where $f^{*}(U)=\bigcup_{u \in U} f(U)$ ).

Define two such maps $f_{1}^{*}, f_{2}^{*}$ to be equivalent if $f_{1}^{*}(x) \sim f_{2}^{*}(x)$ (i.e. $f_{1}^{*}(x) \triangleleft f_{2}^{*}(x)$ and $\left.f_{2}^{*}(x) \varangle f_{1}^{*}(x)\right)$ for all $x \in L$. A continuous map $f:(M, \varangle) \rightarrow(L, \triangleleft)$ is an equivalence class of such maps $f^{*}: L \rightarrow \mathcal{P}(M) .^{24}$

Our main interest in continuous maps lies in the following result. ${ }^{25}$

Proposition 19. Each continuous map $f:(M, \varangle) \rightarrow(L, \triangleleft)$ is equivalent to a frame map $\mathcal{F}(f): \mathcal{F}(L, \triangleleft) \rightarrow \mathcal{F}(M, \varangle)$, given by

$$
\mathcal{F}(f): U \mapsto \mathcal{A} f^{*}(U)
$$

All results in this subsection may be internalized in any topos; for example, a covering relation on an internal meet semilattice $L$ in a topos $\mathcal{T}$ is simply a subobject $\triangleleft$ of $L \times \Omega^{L}$, where $\Omega$ is the subobject classifier in $\mathcal{T}$. The defining properties of a covering relation are then interpreted in the internal language of $\mathcal{T}$. Proposition 19 holds in this generality, since its proof is constructive; see especially [3].

\footnotetext{
22 This even works in case that $L$ is just a set preordered by $x \leqslant y$ when $f(x) \leqslant f(y)$.

23 If $L$ and $M$ have top elements $\top_{L}$ and $\top_{M}$, respectively, then this condition may be replaced by $f^{*}\left(\top_{L}\right)=$ $\top_{M}$.

24 Instead of taking equivalence classes, one could demand as a fourth condition that $f^{*}(x)=\mathcal{A} f^{*}(x)$ for all $x \in L$.

25 In fact, one may extend this into an equivalence $\mathcal{F}$ between the category of formal topologies and the category of frames. A formal topology is a generalization of the above triples $(L, \leqslant, \triangleleft)$, where $\leqslant$ is merely required to be a preorder. In this more general case, the axioms on the cover relation $\triangleleft$ take a slightly different form. See $[8,69]$.
} 
A.1. Localization of the spectrum. We now consider some applications pertinent to the main body of the paper. First, we return to the Gelfand spectrum in Subsect. 2.2. In its presentation by means of generators and relations, Eqs. (18)-(22) play a different role from the regularity rule (23), and we will treat the latter separately. First, for an arbitrary unital commutative $\mathrm{C}^{*}$-algebra $A$ in some topos, consider the distributive lattice $L_{A}$ freely generated by the formal symbols $\mathrm{D}_{a}, a \in A_{\mathrm{sa}}$ (i.e. $a$ is a variable of type $\left.A_{\mathrm{sa}}\right)$, subject to the relations (18)-(22). As shown in [27,31], $L_{A}$ can be described more explicitly, as follows.

Let $A^{+}:=\left\{a \in A_{\text {sa }} \mid a \geq 0\right\}$. Define $p \preccurlyeq q$ iff there exists $n \in \mathbb{N}$ such that $p \leqslant n q$. Define $p \approx q$ iff $p \preccurlyeq q$ and $q \preccurlyeq p$. The lattice operations on $A$ respect $\approx$ and hence $A^{+} / \approx$ is a lattice. We then have

$$
L_{A} \cong A^{+} / \approx .
$$

The image of the generator $\mathrm{D}_{a}$ in $L_{A}$, seen as an element of $A^{+} / \approx$, may also be described explicitly: decomposing $a \in A_{\mathrm{sa}}$ as $a=a^{+}-a^{-}$with $a^{ \pm} \in A^{+}$in the usual way, under the isomorphism (70) this image coincides with the equivalence class $\left[a^{+}\right]$in $A^{+} / \approx$. In explicit computations $[23,47]$, one may therefore simply identify $L_{A}$ with $A^{+} / \approx$ and $\mathrm{D}_{a}$ (seen as an element of $L_{A}$ ) with $\left[a^{+}\right]$, respectively. Such computations are also greatly facilitated by the following 'locality' theorem.

Theorem 20. For each $C \in \mathcal{C}(A)$ one has

$$
\underline{L}_{\underline{A}}(C)=L_{C},
$$

where the right-hand side is simply defined in Sets (where it may be computed through (70)). Furthermore, if $C \subseteq D$, then the map $\underline{L}_{A}(C) \rightarrow \underline{L}_{A}(D)$ given by the functoriality of $\underline{L}_{A}$ simply maps each generator $D_{c}$ for $c \in \bar{C}_{\mathrm{sa}}$ to the same generator for the spectrum of $D$ (this is well defined because $c \in D_{\mathrm{sa}}$, and this inclusion preserves the relations (18)-(22)); we write this as $L_{C} \hookrightarrow L_{D}$.

A proof of this theorem by explicit computation may be found in [22, Thm. 5.2.3]. Here, we give an alternative proof, which requires some familiarity with geometric logic $[51,63,80] .{ }^{26}$ It relies on the following lemmas.

Lemma 21. Let $\mathbb{T}$ be a geometric theory. For any category $\mathbf{C}$, there is an isomorphism of categories $\operatorname{Mod}\left(\mathbb{T}\right.$, Sets $\left.{ }^{\mathrm{C}}\right) \cong \operatorname{Mod}(\mathbb{T}$, Sets $)$.

Here $\operatorname{Mod}(\mathbb{T}, \mathcal{T})$ is the category of $\mathbb{T}$-models in $\mathcal{T}{ }^{27}$ This lemma may be found in [51, Cor. D.1.2.14].

Lemma 22. The lattice $L_{A}$ generating the spectrum of an internal commutative $C^{*}$-algebra $A$ is preserved under inverse images of geometric morphisms.

${ }^{26}$ Further to our remarks in Subsect. 1.4 on geometric propositional logic, we recall that a geometric predicate logic is a theory whose formulae are as described there (where the atomic formulae may now involve relations and equalities and all the usual structures allowed in first-order logic as well), now also involving finitely many free variables $x=\left(x_{1}, \ldots, x_{n}\right)$, and the existential quantifier $\exists$, with axioms taking the form $\forall x: \varphi(x) \rightarrow \psi(x)$. Geometric formulae form an important class of logical formulae, because they are precisely the ones whose truth value is preserved by inverse images of geometric morphisms between topoi. From their syntactic form alone, it follows that their interpretation in the external language is determined locally.

27 This lemma is, in fact, valid for any topos $\mathcal{E}$ replacing Sets; Johnstone's proof just relies on the fact that the functor $\mathrm{ev}_{C}: \mathcal{E}^{\mathbf{C}} \rightarrow \mathcal{E}$ that evaluates at $C \in \mathbf{C}$ is (the inverse image part of) a geometric morphism. The stated generalization follows because the functor $\left(\mathrm{ev}_{C}\right)_{*}: \mathcal{E} \rightarrow \mathcal{E}^{\mathbf{C}}$ given by $\left(\operatorname{ev}_{C}\right)_{*}(S)=S^{\mathbf{C}(-, C)}$ determines the direct image part [63, Exercise VII.10.1]. 
To prove the second lemma, we first use the characterization of the real part $A_{\mathrm{sa}}$ of a commutative $\mathrm{C}^{*}$-algebra $A$ as an f-algebra over the rationals (see Definition 9). Moreover, the spectrum of a $C^{*}$-algebra coincides with the spectrum of the f-algebra of its self-adjoint elements [29]. We claim that the theory of f-algebras is geometric. First, we observe that an f-algebra is precisely a uniquely divisible lattice ordered ring [27, p. 151], since unique divisibility turns a ring into a $\mathbb{Q}$-algebra. The definition of a lattice ordered ring is algebraic: it can be written using equations only. The theory of torsionfree rings, i.e. $\left(n x=0 \vdash_{x} x=0\right)$ for all $n>0$, is also algebraic. The theory of divisible rings is obtained by adding infinitely many geometric axioms $\top \vdash_{x} \exists_{y} n y=x$, one for each $n>0$, to the algebraic theory of rings. A torsion-free divisible ring is the same as a uniquely divisible ring: Suppose that $n y=x$ and $n z=x$, then $n(y-z)=0$, and so $y-z=0$. We conclude that the theory of uniquely divisible lattice ordered rings, i.e. f-algebras, is geometric. In particular, $A_{\text {sa }}$ and hence $A^{+}$are definable by a geometric theory. Secondly, the relation $\approx$ in $(70)$ is defined by an existential quantification, so that the generating lattice $A^{+} / \approx$ - and hence by (70) also $L_{A}$-is preserved under inverse images of geometric morphisms. This proves Lemma 22.

Combining Lemma 22 with Lemma 21, we obtain (71) and hence Theorem 20.

For later we use, we put an important property of $L_{A}$ on record.

Definition 23. A distributive lattice is normal if for all $b_{1}, b_{2}$ such that $b_{1} \vee b_{2}=\top$ there are $c_{1}, c_{2}$ such that $c_{1} \wedge c_{2}=\perp$ and $c_{1} \vee b_{1}=\top$ and $c_{2} \vee b_{2}=\top$. A distributive lattice is called strongly normal if for all $a, b$ there exist $x, y$ such that $a \leqslant b \vee x$ and $b \leqslant a \vee y$ and $x \wedge y=\perp$.

Lemma 24. The lattice $L_{A}$ is strongly normal, and hence normal.

This lemma is due to Coquand [27, Thm. 1.11], but we give a proof.

Proof. First, every strongly normal lattice is normal. To prove this, let $b_{1} \vee b_{2}=\top$ and choose $x, y$ such that $b_{1} \leqslant b_{2} \vee x, b_{2} \leqslant b_{1} \vee y$, and $x \wedge y=\perp$. Then $\top \leqslant b_{1} \vee b_{2} \leqslant$ $\left(b_{2} \vee x\right) \vee b_{2}=b_{2} \vee x$. Similarly, $\top=b_{1} \vee y$.

Second, to check that $L_{A}$ is strongly normal, it is enough to verify the defining property on the generators $\mathrm{D}_{a}$. So we pick $a, b$ in $A_{\mathrm{sa}}$. Then one has $\mathrm{D}_{a} \leqslant \mathrm{D}_{a-b} \vee \mathrm{D}_{b}$, $\mathrm{D}_{b} \leqslant \mathrm{D}_{b-a} \vee \mathrm{D}_{a}$, and $\mathrm{D}_{a-b} \wedge \mathrm{D}_{b-a}=\perp$.

We now turn to the relation (23), which is to be imposed on $L_{A}$. It turns out that 23 is a special case of a relation that can be defined on any distributive lattice $L$ by $x \ll y$ iff there exists $z$ such that $x \wedge z=\perp$ and $y \vee z=\top .^{28}$

Lemma 25. For all $D_{a}, D_{b} \in L_{A}$, the following are equivalent: ${ }^{29}$

1. There exists $D_{c} \in L_{A}$ such that $D_{c} \vee D_{a}=\top$ and $D_{c} \wedge D_{b}=\perp$;

2. There exists $q>0$ such that $D_{b} \leqslant D_{a-q}$.

Proof. $1 \Rightarrow 2$ : By [27, Cor 1.7] there exists $q>0$ such that $\mathrm{D}_{c-q} \vee \mathrm{D}_{a-q}=\top$. Hence $\mathrm{D}_{c} \vee \mathrm{D}_{a-q}=\mathrm{T}$, so $\mathrm{D}_{b}=\mathrm{D}_{b} \wedge\left(\mathrm{D}_{c} \vee \mathrm{D}_{a-q}\right)=\mathrm{D}_{b} \wedge \mathrm{D}_{a-q} \leqslant \mathrm{D}_{a-q}$.

$2 \Rightarrow 1$ : Choose $\mathrm{D}_{c}:=\mathrm{D}_{q-a}$.

28 Banaschewski and Mulvey write that $x$ is 'rather below' $y$ [7], whereas Johnstone [48] says that $x$ is 'well inside' $y$. The notation « is usually reserved for the so-called 'way below' relation, but this relation coincides with the 'well inside' relation on compact regular locales (see [48, p.303] and Theorem 27), so we feel entitled to identify them notationally.

29 In what follows, one may take $q>0$ either in $\mathbb{Q}$ or in $\mathbb{R}$. 
Hence in what follows we write

$$
\mathrm{D}_{b} \ll \mathrm{D}_{a} \text { iff } \exists_{q>0} \mathrm{D}_{b} \leqslant \mathrm{D}_{a-q},
$$

and note with Coquand [27] that in view of the above lemma the relation (23) just states that the frame $\mathcal{O}(\Sigma)$ is regular. ${ }^{30}$ This leads to the following description.

For any distributive lattice $L$, an ideal $I \in \operatorname{Idl}(L)$ is called regular if $I \supseteq \Downarrow x$ implies $x \in I$, where

$$
\downarrow x=\{y \in L \mid y \ll x\} .
$$

Expressed in logical language, $I$ is therefore a regular ideal if

$$
\forall y \in L(y \ll x \Rightarrow y \in I) \Rightarrow x \in I,
$$

and hence one has the frame $\operatorname{RIdl}(L)$ of regular ideals of $L$, defined by

$$
\operatorname{RIdl}(L)=\left\{U \in \operatorname{Idl}(L) \mid\left(\forall_{y \in L} y \ll x \Rightarrow y \in U\right) \Rightarrow x \in U\right\} ;
$$

for the sake of completeness, $U \in \operatorname{Idl}(L)$ as a predicate on $\mathcal{P}(L)$ stands for $\perp \equiv 0 \in U$ and

$$
\begin{aligned}
x \in U, y \leqslant x & \Rightarrow y \in U ; \\
x, y \in U & \Rightarrow x \vee y \in U .
\end{aligned}
$$

Any ideal $U \in \operatorname{Idl}(L)$ can be turned into a regular ideal $\mathcal{A} U$ by means of the closure operation $\mathcal{A}: D L \rightarrow D L$ defined by [24]

$$
\mathcal{A} U=\{x \in L \mid \forall y \in L \quad y \ll x \Rightarrow y \in U\},
$$

and the canonical map $f: L \rightarrow \operatorname{RIdl}(L)$ is given in terms of (78) by (67).

Combining Theorem 27 in [24] (which states that the regular ideals in a normal distributive lattice form a compact regular frame) with Theorem 1.11 in [27] (which applies this to the case at hand), we finally obtain:

Theorem 26. The Gelfand spectrum $\mathcal{O}(\Sigma)$ of a commutative unital $C^{*}$-algebra $A$ is isomorphic (as a frame) to the frame of all regular ideals of $L_{A}$, i.e.

$$
\mathcal{O}(\Sigma) \cong\left\{U \in \operatorname{Idl}\left(L_{A}\right) \mid\left(\forall_{D_{b} \in L_{A}} D_{b} \ll D_{a} \Rightarrow D_{b} \in U\right) \Rightarrow D_{a} \in U\right\} .
$$

In this realization, the canonical map $f: L_{A} \rightarrow \mathcal{O}(\Sigma)$ is given by

$$
f\left(D_{a}\right)=\left\{D_{c} \in L_{A} \mid \forall D_{b} \in L_{A} D_{b} \ll D_{c} \Rightarrow D_{b} \leqslant D_{a}\right\} .
$$

By construction, we then have

$$
f\left(\mathrm{D}_{a}\right) \leqslant \bigvee\left\{f\left(\mathrm{D}_{a-q}\right) \mid q>0\right\} .
$$

For later use, also note that (80) implies

$$
f\left(\mathrm{D}_{a}\right)=\top \Leftrightarrow \mathrm{D}_{a}=\top .
$$

30 See [48, III.1.1] for this notion. Recall that by the general theory of Banaschewski and Mulvey [7], the spectrum has to be a compact regular frame. 
We may now equip $L_{A}$ with the covering relation defined by (68), given (79) and the ensuing map (80). ${ }^{31}$ Consequently, by Proposition 17 one has

$$
\mathcal{O}(\Sigma) \cong \mathcal{F}\left(L_{A}, \triangleleft\right)
$$

This description becomes computable by the following two results.

Theorem 27. In any topos, the covering relation $\triangleleft$ on $L_{A}$ defined by (68) with (79) and (80) is given by $D_{a} \triangleleft U$ iff for all $q>0$ there exists a (Kuratowski) finite $U_{0} \subseteq U$ such that $D_{a-q} \leqslant \bigvee U_{0}$. (If $U$ is directed, this means that there exists $D_{b} \in U$ such that $D_{a-q} \leqslant D_{b}$.)

Proof. The easy part is the " $\Leftarrow$ " direction: from (81) and the assumption we have $f\left(\mathrm{D}_{a}\right) \leqslant \bigvee f(U)$ and hence $\mathrm{D}_{a} \triangleleft U$ by definition of the covering relation.

In the opposite direction, assume $\mathrm{D}_{a} \triangleleft U$ and take some $q>0$. From (the proof of) Lemma 25, $\mathrm{D}_{a} \vee \mathrm{D}_{q-a}=\top$, hence $\bigvee f(U) \vee f\left(\mathrm{D}_{q-a}\right)=\top$. Since $\mathcal{O}(\Sigma)$ is compact, there is a finite $U_{0} \subset U$ for which $\bigvee f\left(U_{0}\right) \vee f\left(\mathrm{D}_{q-a}\right)=\top$, so that by (82) we have $\mathrm{D}_{b} \vee \mathrm{D}_{q-a}=\top$, with $\mathrm{D}_{b}=\bigvee U_{0}$. By (19) we have $\mathrm{D}_{a-q} \wedge \mathrm{D}_{q-a}=\perp$, and hence

$$
\mathrm{D}_{a-q}=\mathrm{D}_{a-q} \wedge \top=\mathrm{D}_{a-q} \wedge\left(\mathrm{D}_{b} \vee \mathrm{D}_{q-a}\right)=\mathrm{D}_{a-q} \wedge \mathrm{D}_{b} \leqslant \mathrm{D}_{b}=\bigvee U_{0}
$$

Thus we have two alternative expressions for the spectrum:

$$
\begin{aligned}
\mathcal{O}(\Sigma) & \cong\left\{U \in \operatorname{Idl}\left(L_{A}\right) \mid \forall_{q>0} \mathrm{D}_{a-q} \in U \Rightarrow \mathrm{D}_{a} \in U\right\}, \\
& \cong\left\{U \in \mathcal{P}\left(L_{A}\right) \mid \mathrm{D}_{a} \triangleleft U \Rightarrow \mathrm{D}_{a} \in U\right\} .
\end{aligned}
$$

The first follows from (79), the second from (64) and (83).

To apply this to our functor topos $\mathcal{T}(A)$, we apply the Kripke-Joyal semantics for the internal language of the topos $\mathcal{T}(A)$ (see [63, Sect. VI.7], whose notation we will use, and [12, Sect. 6.6]) to the statement $\mathrm{D}_{a} \triangleleft U$. This is a formula $\phi$ with two free variables, namely $\mathrm{D}_{a}$ of type $L_{A}$, and $U$ of type $\mathcal{P}\left(L_{A}\right) \equiv \Omega^{L_{A}}$. Hence in the forcing statement $C \Vdash \phi(\alpha)$ in $\mathcal{T}(A)$, we have to insert

$$
\alpha \in\left(\underline{L}_{A} \times \underline{\Omega}^{\underline{L}} \underline{A}\right)(C) \cong L_{C} \times \operatorname{Sub}\left(\underline{L}_{A \mid \uparrow C}\right)
$$

where $\underline{L}_{\underline{A} \mid \uparrow C}$ is the restriction of the functor $\underline{L}_{A}: \mathcal{C}(A) \rightarrow$ Sets to $\uparrow C \subset \mathcal{C}(A)$. Here we have used (71), as well as the isomorphism [63, Sect. II.8]

$$
\underline{\Omega}^{\underline{L}} \underline{A}(C) \cong \operatorname{Sub}\left(\underline{L}_{A \mid \uparrow C}\right) .
$$

Consequently, we have $\alpha=\left(D_{c}, \underline{U}\right)$, where $D_{c} \in L_{C}$ for some $c \in C_{\text {sa }}$ (note the change of typefont between the formal variable $D_{a}$ and the actual element $D_{c}$ ) and $\underline{U}: \uparrow C \rightarrow$ Sets is a subfunctor of $\underline{L}_{A \mid \uparrow C}$. In particular, $\underline{U}(D) \subseteq L_{D}$ is defined whenever $\bar{D} \supseteq C$, and the subfunctor condition on $\underline{U}$ simply boils down to $\underline{U}(D) \subseteq \underline{U}(E)$ whenever $C \subseteq D \subseteq E$.

\footnotetext{
31 Alternatively, writing $\mathrm{D}_{a} \triangleleft_{0} U$ iff $U \supseteq \downarrow_{a}$, the covering relation $\triangleleft$ is inductively generated by $\triangleleft_{0}$, as explained in $[28,79]$. The triple $\left(L_{A}, \leqslant, \triangleleft_{0}\right)$ is a flat site as defined in [79].
} 
Corollary 28. In the topos $\mathcal{T}(A)$ the cover $\triangleleft$ of Theorem 27 may be computed locally, in the sense that for any $C \in \mathcal{C}(A), D_{c} \in L_{C}$ and $\underline{U} \in \operatorname{Sub}\left(\underline{L}_{A \mid \uparrow C}\right)$, one has

$$
C \Vdash D_{a} \triangleleft U\left(D_{c}, \underline{U}\right) \text { iff } D_{c} \triangleleft_{C} \underline{U}(C),
$$

in that for all $q>0$ there exists a finite $U_{0} \subseteq \underline{U}(C)$ such that $D_{c-q} \leqslant \bigvee U_{0}$.

Proof. For simplicity, assume that $\bigvee U_{0} \in U$, so that we may replace $U_{0}$ by $\mathrm{D}_{b}=\bigvee U_{0}$; the general case is analogous. We then have to inductively analyze the formula $\mathrm{D}_{a} \triangleleft U$, which, under the stated assumption, in view of Theorem 27 may be taken to mean

$$
\forall_{q>0} \exists_{D_{b} \in L_{A}}\left(\mathrm{D}_{b} \in U \wedge \mathrm{D}_{a-q} \leqslant \mathrm{D}_{b}\right) .
$$

We now infer from the rules for Kripke-Joyal semantics in a functor topos that: ${ }^{32}$

1. $C \Vdash\left(D_{a} \in U\right)\left(D_{c}, \underline{U}\right)$ iff for all $D \supseteq C$ one has $D_{c} \in \underline{U}(D)$; since $\underline{U}(C) \subseteq \underline{U}(D)$, this happens to be the case iff $D_{c} \in \underline{U}(C)$.

2. $C \Vdash\left(D_{b} \leqslant D_{a}\right)\left(D_{c^{\prime}}, D_{c}\right)$ iff $D_{c^{\prime}} \leqslant D_{c}$ in $L_{C}$.

3. $C \Vdash\left(\exists_{D_{b} \in L_{A}} \mathrm{D}_{b} \in U \wedge \mathrm{D}_{a-q} \leqslant \mathrm{D}_{b}\right)\left(D_{c}, \underline{U}\right)$ iff there is $D_{c^{\prime}} \in \underline{U}(C)$ such that $D_{c-q} \leqslant D_{c^{\prime}}$.

4. $C \Vdash\left(\forall_{q>0} \exists_{D_{b} \in L_{A}} \mathrm{D}_{b} \in U \wedge \mathrm{D}_{a-q} \leqslant \mathrm{D}_{b}\right)\left(D_{c}, \underline{U}\right)$ iff for all $D \supseteq C$ and all $q>0$ there is $D_{d} \in \underline{U}(D)$ such that $D_{c-q} \leqslant D_{d}$, where $D_{c} \in L_{C}$ is seen as an element of $L_{D}$ through the injection $L_{C} \hookrightarrow L_{D}$ of Theorem 20 , and $\underline{U} \in \operatorname{Sub}\left(\underline{L}_{A \mid \uparrow C}\right)$ is seen as an element of $\operatorname{Sub}\left(\underline{L}_{A \mid \uparrow D}\right)$ by restriction. This, however, is true at all $D \supseteq C$ iff it is true at $C$, because $\underline{U}(C) \subseteq \underline{U}(D)$ and hence one can take $D_{d}=D_{c^{\prime}}$ for the $D_{C^{\prime}} \in L_{C}$ that makes the condition true at $C$.

This brings us to our recipe for computing the spectrum in $\mathcal{T}(A)$ locally:

Theorem 29. The spectrum $\mathcal{O}(\underline{\Sigma})$ of $\underline{A}$ in $\mathcal{T}(A)$ can be computed as follows:

1. At $C \in \mathcal{C}(A)$, the set $\mathcal{O}(\underline{\Sigma})(C)$ consists of those subfunctors $\underline{U} \in \operatorname{Sub}\left(\underline{L}_{A \mid \uparrow C}\right)$ such that for all $D \supseteq C$ and all $D_{d} \in L_{D}$ one has $D_{d} \triangleleft_{D} \underline{U}(D) \Rightarrow D_{d} \in \underline{U}(D)$.

2. In particular, at $\mathbb{C} \cdot 1$, the set $\mathcal{O}(\underline{\Sigma})(\mathbb{C} \cdot 1)$ consists of those subfunctors $\underline{U} \in \operatorname{Sub}\left(\underline{L}_{A}\right)$ such that for all $C \in \mathcal{C}(A)$ and all $D_{c} \in L_{C}$ one has $D_{c} \triangleleft_{C} \underline{U}(C) \Rightarrow D_{c} \in \underline{U}(\bar{C})$.

3. The condition that $\underline{U}=\left\{\underline{U}(C) \subseteq L_{C}\right\}_{C \in \mathcal{C}(A)}$ be a subfunctor of $\underline{L}_{\underline{A}}$ comes down to the requirement that $\underline{U}(C) \subseteq \underline{U}(D)$ whenever $C \subseteq D$.

4. The map $\mathcal{O}(\underline{\Sigma})(C) \rightarrow \mathcal{O}(\underline{\Sigma})(D)$ given by the functoriality of $\mathcal{O}(\Sigma)$ whenever $C \subseteq D$ is given by truncating an element $\underline{U}: \uparrow C \rightarrow$ Sets of $\mathcal{O}(\underline{\Sigma})(C)$ to $\uparrow D$.

5. The external description of $\mathcal{O}(\underline{\Sigma})$ is the frame map

$$
\pi_{\Sigma}^{*}: \mathcal{O}(\mathcal{C}(A)) \rightarrow \mathcal{O}(\underline{\Sigma})(\mathbb{C} \cdot 1)
$$

given on the basic opens $\uparrow D \in \mathcal{O}(\mathcal{C}(A))$ by

$$
\begin{aligned}
\pi_{\Sigma}^{*}(\uparrow D)=\chi_{\uparrow D}: E & \mapsto \top(E \supseteq D) ; \\
E & \mapsto \perp(E \nsupseteq D),
\end{aligned}
$$

where the top and bottom elements $\top, \perp$ at E are given by $\left\{L_{E}\right\}$ and $\emptyset$, respectively.

\footnotetext{
32 The first one follows from [12, Prop. 6.6.10] and a routine computation. The others are obvious from either [63, Sect. VI.7] or [12, Sect. 6.6].
} 
Proof. By $(85), \mathcal{O}(\underline{\Sigma})$ is the subobject of $\underline{\Omega}^{\underline{L}} \underline{A}$ defined by the formula $\phi$ given by

$$
\forall \mathrm{D}_{a} \in L_{A} \mathrm{D}_{a} \triangleleft U \Rightarrow \mathrm{D}_{a} \in U
$$

whose interpretation in $\mathcal{T}(A)$ is an arrow from $\underline{\Omega} \underline{\underline{L}} \underline{\underline{A}}$ to $\underline{\Omega}$. In view of (86), we may identify an element $\underline{U} \in \mathcal{O}(\underline{\Sigma})(C)$ with a subfunctor of $\underline{L}_{A \mid \uparrow C}$, and by (90) and Kripke-Joyal semantics in functor topoi (see, in particular, [63, Sect. IV.7]), we have $\underline{U} \in \mathcal{O}(\underline{\Sigma})(C)$ iff $C \Vdash \phi(\underline{U})$, with $\phi$ given by (90). Unfolding this using the rules for Kripke-Joyal semantics and using Corollary 28 (including part 1 of its proof), we find that $\underline{U} \in \mathcal{O}(\underline{\Sigma})(C)$ iff

$$
\forall_{D \supseteq C} \forall_{D_{d} \in L_{D}} \forall_{E \supseteq D} D_{d} \triangleleft_{E} \underline{U}(E) \Rightarrow D_{d} \in \underline{U}(E),
$$

where $D_{d}$ is regarded as an element of $L_{E}$. This condition, however, is equivalent to the apparently weaker condition

$$
\forall_{D \supseteq C} \forall_{D_{d} \in L_{D}} D_{d} \triangleleft_{D} \underline{U}(D) \Rightarrow D_{d} \in \underline{U}(D) ;
$$

condition (91) clearly implies (92), but the latter applied at $D=E$ actually implies the first, since $D_{d} \in L_{D}$ also lies in $L_{E}$.

Items 2 to 4 are now obvious, and the last follows by the explicit prescription for the external description of frames recalled in Subsect. 1.7. Note that each $\mathcal{O}(\underline{\Sigma})(C)$ is a frame in Sets, inheriting the frame structure of the ambient frame $\operatorname{Sub}\left(\underline{L}_{A \mid \uparrow C}\right)$.

An equivalent way to compute the spectrum, which derives from (84) rather than (85), is as follows: $\mathcal{O}(\underline{\Sigma})(\mathbb{C} \cdot 1)$ (and similarly all the other $\mathcal{O}(\underline{\Sigma})(C)$ ) consists of those subfunctors $\underline{U} \in \operatorname{Sub}\left(\underline{L}_{A}\right)$ such that for all $C \in \mathcal{C}(A), \underline{U}(C)$ is a regular ideal in $L_{C}$.

To prove this, according to (84) the formula expressing that $U \in \mathcal{P}\left(L_{A}\right)$ be a regular ideal is

$$
U \in \operatorname{Idl}(L) \wedge \forall_{\mathrm{D}_{a} \in L_{A}} \forall_{q>0} \mathrm{D}_{a-q} \in U \Rightarrow \mathrm{D}_{a} \in U,
$$

where the condition $U \in \operatorname{Idl}(L)$ is spelled out in (76) and (77). The locality of this first condition and of the conjunction in (93) being almost trivial, we concentrate on the second term, calling it $\phi$ as usual. We then find that $\mathbb{C} \cdot 1 \Vdash \phi(\underline{U})$ iff for all $C \in \mathcal{C}(A)$, all $D_{c} \in \underline{U}(C)$, and all $D \supseteq C$ one has: if $D_{c-q} \in \underline{U}(E)$ for all $q>0$ at all $E \supseteq D$, then $D_{c} \in \underline{U}(D)$. Now the antecedent automatically holds at all $E \supseteq D$ iff it holds at $D$, and similarly the if ...then statement holds at all $D \supseteq C$ if it holds at $C$.

A.2. Daseinisation map. Our next aim is to construct the Daseinisation map (44), which, read as a frame map, for fixed $a \in A_{\mathrm{sa}}$ is

$$
\underline{\delta}(a)^{-1}: \mathcal{O}(\underline{\mathbb{R}}) \rightarrow \mathcal{O}(\underline{\Sigma}) .
$$

We will use the realization (83) of the spectrum $\mathcal{O}(\underline{\Sigma})$ of $\underline{A}$ as the frame $\mathcal{F}\left(\underline{L}_{A}, \unlhd\right)$ defined in the preceding subsection. The second frame we deal with is that of the interval domain $\mathcal{O}(\mathbb{I R}), c f$. Subsect. 5.1. Following [69], we construct the interval domain as a frame $\mathcal{F}(\mathbb{Q} \times<\mathbb{Q}, \varangle)$ defined by a covering relation. Here the pertinent meet semilattice $\mathbb{Q} \times<\mathbb{Q}$ consists of pairs $(p, q) \in \mathbb{Q} \times \mathbb{Q}$ with $p<q$, ordered by inclusion (i.e. $(p, q) \leqslant\left(p^{\prime}, q^{\prime}\right)$ iff $p^{\prime} \leqslant p$ and $\left.q \leqslant q^{\prime}\right)$, with a bottom element $\perp$ added. The covering relation $\varangle$ is defined by $\perp \varangle U$ for all $U$ and $(p, q) \varangle U$ iff for all rational $p^{\prime}, q^{\prime}$ with 
$p<p^{\prime}<q^{\prime}<q$ there exists $\left(p^{\prime \prime}, q^{\prime \prime}\right) \in U$ with $\left(p^{\prime}, q^{\prime}\right) \leqslant\left(p^{\prime \prime}, q^{\prime \prime}\right)$. In Sets one easily verifies the frame isomorphism

$$
\mathcal{F}(\mathbb{Q} \times<\mathbb{Q}, \varangle) \cong \mathcal{O}(\mathbb{I R}),
$$

so that, in particular, we may regard $\mathcal{O}(\mathbb{R})$ as a subset of the power set $\mathcal{P}(\mathbb{Q} \times<\mathbb{Q})$.

Proposition 30. The functor $\mathcal{O}(\underline{\mathbb{R}})$ internalizing the interval domain in $\mathcal{T}(A)$ is given by

$$
\mathcal{O}(\underline{\mathbb{I R}}) \cong \mathcal{F}(\underline{\mathbb{Q} \times<\mathbb{Q}}, \leq) .
$$

Explicitly, we have

$$
\mathcal{O}(\underline{\mathbb{R}})(\mathbb{C} \cdot 1) \cong\{\underline{S} \in \operatorname{Sub}(\underline{\mathbb{Q}} \times<\mathbb{Q}) \mid \underline{S}(C) \in \mathcal{O}(\mathbb{I} \mathbb{R}) \text { for all } C \in \mathcal{C}(A)\},
$$

where $\mathcal{O}(\mathbb{I} \mathbb{R}) \subset \mathcal{P}(\mathbb{Q} \times<\mathbb{Q})$ through (95), as just explained. Furthermore, $\mathcal{O}(\mathbb{R})(C)$ is the truncation of (97) to $\uparrow C$ (cf. Theorem 29), and the functorial map $\mathcal{O}(\mathbb{\mathbb { R }})(C) \rightarrow$ $\mathcal{O}(\mathbb{R})(D)$ whenever $C \subseteq D$ is given by truncation. Finally, the external description of $\mathcal{O}(\underline{\mathbb{R}})$ is given by the frame map

$$
\pi_{\mathbb{R}}^{*}: \mathcal{O}(\mathcal{C}(A)) \rightarrow \mathcal{O}(\underline{\mathbb{R}})(\mathbb{C} \cdot 1),
$$

where $\pi_{\mathbb{R}}^{*}$ is given by a formula similar to (89).

Proof. This follows from a computation analogous to but simpler than the proof of Theorem 29, combined with the remark following (47) and the observation that the condition that $\underline{S}: \mathcal{C}(A) \rightarrow \mathcal{P}(\mathbb{Q} \times<\mathbb{Q})$ in the right-hand side of (97) be a subfunctor of $\underline{\mathbb{Q}} \times<\mathbb{Q}$ means that we may identify $\underline{S}$ with a monotone function from $\mathcal{C}(A)$ to $\mathcal{O}(\mathbb{I} \mathbb{R})$.

We now give the external description

$$
\delta(a)^{-1}: \mathcal{O}(\underline{\mathbb{R}})(\mathbb{C} \cdot 1) \rightarrow \mathcal{O}(\underline{\Sigma})(\mathbb{C} \cdot 1)
$$

of our Daseinisation map (94). In view of (83) and (96), we will define (99) as a frame map

$$
\delta(a)^{-1}: \mathcal{F}\left(\underline{\mathbb{Q}} \times_{<} \mathbb{Q}, \unlhd\right)(\mathbb{C} \cdot 1) \rightarrow \mathcal{F}\left(\underline{L}_{\underline{A}}, \unlhd\right)(\mathbb{C} \cdot 1) .
$$

Internalizing Proposition 19 to $\mathcal{T}(A)$, we proceed by constructing a continuous map

$$
\underline{d}(a):\left(\underline{L}_{\underline{A}}, \unlhd\right) \rightarrow(\underline{\mathbb{Q} \times<\mathbb{Q}}, \unlhd)
$$

for in that case we may put

$$
\delta(a)^{-1}=\mathcal{F}(\underline{d}(a))(\mathbb{C} \cdot 1) .
$$

By definition, as a map in the functor topos $\mathcal{T}(A)$ the continuous map $\underline{d}(a)$ is a natural transformation

$$
\underline{d}(a)^{*}: \underline{\mathbb{Q} \times<\mathbb{Q}} \rightarrow \underline{\mathcal{P}\left(L_{A}\right)}=\underline{\Omega}^{\underline{L_{A}}}
$$


with components $\underline{d}(a)_{C}^{*}: \underline{\mathbb{Q}} \times_{<} \mathbb{Q}(C) \rightarrow \underline{\Omega}^{\underline{L}} \underline{A}(C)$. One has $\underline{\mathbb{Q}} \times_{<} \mathbb{Q}(C) \cong \mathbb{Q} \times<\mathbb{Q}$, so by (86) the $\underline{d}(a)_{C}^{*}$ are maps

$$
\underline{d}(a)_{C}^{*}: \mathbb{Q} \times<\mathbb{Q} \rightarrow \operatorname{Sub}\left(\underline{L}_{A \mid \uparrow C}\right) .
$$

By naturality, $\underline{d}(a)_{C}^{*}$ is determined by $\underline{d}(a)_{\mathbb{C} \cdot 1}^{*}: \mathbb{Q} \times<\mathbb{Q} \rightarrow \operatorname{Sub}\left(\underline{L}_{A}\right)$ as

$$
\underline{d}(a)_{C}^{*}(r, s)(D)=\underline{d}(a)_{\mathbb{C} \cdot 1}^{*}(r, s)(D),
$$

for all $D \supseteq C$, so $\underline{d}(a)^{*}$ is determined by $\underline{d}(a)_{\mathbb{C} \cdot 1}^{*}$. Using the description of the lattice $\underline{L}_{\underline{A}}(C)$ by Theorem 20 , we may now define

$$
\underline{d}(a)_{\mathbb{C} \cdot 1}^{*}(r, s): C \mapsto\left\{D_{f-r} \wedge D_{s-g} \mid f, g \in C_{\mathrm{sa}}, f \leqslant a \leqslant g\right\},
$$

which is indeed a subset of $\underline{L}_{A}(C)=L_{C}$, as required.

Lemma 31. The map (101) defined by (103), (105) and (106) is continuous (in the sense of Definition 18, internalized to $\mathcal{T}(A)$ ).

Proof. First, we claim that $\underline{d}$ is continuous iff each $\underline{d}(a)_{C}$ is. Indeed, with regard to the first condition in Definition 18 this is obvious; for the second $c f$. [63, Prop. I.8.5], and for the third this is true because both covering relations are described locally in $C$ ( $c f$. Corollary 28). By Proposition 19, continuity of $\underline{d}$, in turn, would mean that (100) is well defined as a frame map.

Thus what remains is to verify that each map $\underline{d}(a)_{C}$ is continuous in the sense of Definition 18. This is indeed the case; we spare the readers the details. ${ }^{33}$

We now compute the associated frame map (100). The map (103) induces a map $\operatorname{Sub}(\underline{\mathbb{Q}} \times<\mathbb{Q}) \rightarrow \operatorname{Sub}\left(\underline{\Omega}^{\underline{L}} \underline{A}\right)$ as the left adjoint of the pullback in the opposite direction (see, e.g., [63, Exercise I.10]), which by composition with $\cup$ yields a map $\operatorname{Sub}(\underline{\mathbb{Q}} \times<\mathbb{Q}) \rightarrow \operatorname{Sub}\left(\underline{L}_{A}\right)$. The latter restricts to a map $\mathcal{F}\left(\mathbb{Q} x_{<} \mathbb{Q}, \underline{\Delta}\right)(\mathbb{C} \cdot 1) \rightarrow$ $\mathcal{F}\left(\underline{L}_{A}, \unlhd\right)(\mathbb{C} \cdot 1)$, which by definition is the map (100) and hence gives the external description (99) of our Daseinisation map. ${ }^{34}$ This is a frame map by construction; see Lemma 31 and Proposition 19. The associated locale map $\delta(a): \underline{\Sigma} \rightarrow \underline{\mathbb{R}}$ is our version of the Döring-Isham Daseinisation map. It is unenlightening to write it down explicitly, but we give an appealing special case in Subsect. 5.2.

A.3. Localization of integrals. Finally, to compute the interpretation of the locale of integrals we may proceed analogously to the case of the spectrum. The free distributive lattice satisfying the relations in Sect. 4.2 may alternatively be defined by an entailment relation [24]. Consequently, it suffices to describe when $\wedge A \vdash \vee B$ in the lattice. As proved in [27,30], this holds if a positive combination of elements in $A$ is below a positive combination of elements in $B$ - in symbols, if there are $r_{i}, s_{j}>0$ and $a_{i}$ in $A$ and $b_{j}$ in $B$ such that $\sum r_{i} a_{i} \leqslant \sum s_{j} b_{j}$. This is an existential quantification over finite subsets of an f-algebra. The construction of taking the (Kuratowski) finite powerset is geometric, see e.g. [80]. So existential quantification over it is preserved by geometric morphisms. Applying this to the internal $C^{*}$-algebra and applying Lemma 21 we obtain (cf. Theorem 20):

\footnotetext{
33 These will appear in the PhD Thesis of the first author.

34 According to (69), this map is just the component of $\mathcal{F}(\underline{d}(a))$ at $\mathbb{C} \cdot 1$. This component, however, determines $\mathcal{F}(\underline{d}(a))$ as a whole, since $\mathcal{F}(\underline{d}(a))(C)$ is just the restriction of $\mathcal{F}(\underline{d}(a))(\mathbb{C} \cdot 1)$ to the truncation of each subfunctor $\underline{S}$ in (97) to $\uparrow C$.
} 
Proposition 32. The interpretation of the lattice generating the locale of integrals of the internal $C^{*}$-algebra is given by the functor assigning to each commutative subalgebra $C$ the lattice generating the integrals on $C$. If $C \subseteq D$, then the inclusion maps generators of the lattice for $C$ to generators of the lattice for $D$ and preserves relations. The covering relation for the space of integrals is also interpreted locally.

A similar statement holds for valuations; see [30,81]. Vickers [78] uses a presentation of locales which is similar to formal topology, but which is tailored for geometric reasoning.

\section{B. Related Work}

The present article was to a considerable extent motivated by the fundamental work of Butterfield and Isham [20,21] and Döring and Isham [34-38]. We refrain from a full comparison, but restrict ourselves to what we see as the key points.

As to Butterfield and Isham, our reformulation of the Kochen-Specker Theorem is in their spirit, but we feel our version is more powerful, especially from a logical perspective: our statement that a certain locale has no points has a logical interpretation in terms of (the lack of) models of a certain geometric theory, whereas the original reformulation [20] merely claims that some presheaf lacks global sections (i.e. points).

Compared with Döring and Isham, our overall programme and philosophy, as explained in the Introduction, are quite different from theirs: our ambitions are limited to finding a spatial notion of quantum logic (although we do hope that locales in topoi might provide a generalized concept of space that will be useful in quantum gravity). The principal technical differences between the two approaches lie in our use of:

1. covariant functors (instead of contravariant ones);

2. C*-algebras (instead of von Neumann algebras);

3. locales (instead of Stone spaces);

4. internal reasoning and the associated use of Kripke-Joyal semantics;

5. states as internal integrals and the correspondence between integrals and valuations (i.e. measures defined on open sets). ${ }^{35}$

This has many technical advantages, which has made it possible to obtain our main results (see Subsect. 1.9). Conceptually, the two programs in question overlap to the effect that the Gelfand spectrum $\mathcal{O}(\underline{\Sigma})$ of the Bohrification $\underline{A}$ of $A$ provides a pointfree realization of Döring and Isham's notion of a state object in a topos, whereas the interval domain $\mathcal{O}(\underline{\mathbb{R}})$ realizes their quantity object, again in the sense of pointfree topology internalized to a suitable topos. ${ }^{36}$ These objects are linked by observables, which define arrows from the state object to the quantity object. Thus for each $a \in A_{\mathrm{sa}}$, our Daseinisation $\delta(a): \underline{\Sigma} \rightarrow \underline{\mathbb{R}}$ is an observable in the sense of Döring and Isham. Restricted to the special case $A=B(H)$, our construction resembles the single example of such a topos that both Butterfield, Hamilton and Isham [19] and Döring and Isham $[35,36]$ give, namely that of presheaves over the preorder category of commutative von Neumann subalgebras of $B(H)$ (ordered by inclusion).

Acknowledgement. The authors are indebted to Andreas Döring, Ieke Moerdijk, Chris Mulvey, Isar Stubbe, and Steve Vickers for guidance and useful feedback on talks and earlier drafts of this article. We are exceptionally grateful to the referee of this paper for unusually detailed and helpful comments.

\footnotetext{
35 An analogous external result has meanwhile been found by Döring [33].

36 In fact, our use of pointfree techniques leads to topoi of covariant functors just as inevitably as the more conventional methods in [19-21,34-37] lead to topoi of presheaves.
} 
Open Access This article is distributed under the terms of the Creative Commons Attribution Noncommercial License which permits any noncommercial use, distribution, and reproduction in any medium, provided the original author(s) and source are credited.

\section{References}

1. Aarnes, J.F.: Quasi-states on $C^{*}$-algebras. Trans. Amer. Math. Soc. 149, 601-625 (1970)

2. Abramsky, S., Jung, A.: Domain theory. In: Handbook for Logic in Computer Science. Volume 3. London: Clarendon Press, 1994

3. Aczel, P.: Aspects of general topology in constructive set theory. Ann. Pure Appl. Logic 137, 3-29 (2006)

4. Adelman, M., Corbett, J.V.: A sheaf model for intuitionistic quantum mechanics. Appl. Categ. Struct. 3, 79-104 (1995)

5. Banaschewski, B., Mulvey, C.J.: The spectral theory of commutative $C^{*}$-algebras: the constructive Gelfand-Mazur theorem. Quaest. Math. 23(4), 465-488 (2000)

6. Banaschewski, B., Mulvey, C.J.: The spectral theory of commutative $\mathrm{C}^{*}$-algebras: the constructive spectrum. Quaest. Math. 23(4), 425-464 (2000)

7. Banaschewski, B., Mulvey, C.J.: A globalisation of the Gelfand duality theorem. Ann. Pure Appl. Logic 137(1-3), 62-103 (2006)

8. Battilotti, G., Sambin, G.: Pretopologies and uniform presentation of sup-lattices, quantales and frames. Ann. Pure Appl. Logic. 137, 30-61 (2006)

9. Bell, J.L.: The development of categorical logic. In: Handbook of Philosophical Logic. Volume 12. Berlin-Heidelberg-New York: Springer, 2005

10. Birkhoff, G., von Neumann, J.: The logic of quantum mechanics. Ann. Math. 37(4), 823-843 (1936)

11. Bohr, N.: Discussion with Einstein on epistemological problems in atomic physics. In: Albert Einstein: Philosopher-Scientist, La Salle: Open Court, 1949, pp. 201-241

12. Borceux, F.: Handbook of Categorical Algebra. 3. Categories of sheaves. Volume $\mathbf{5 2}$ of Encyclopedia of Mathematics and its Applications. Cambridge: Cambridge University Press, 1994

13. Brunetti, R., Fredenhagen, K., Verch, R.: The generally covariant locality principle — a new paradigm for local quantum field theory. Commun. Math. Phys. 237(1-2), 31-68 (2003)

14. Bub, J.: Interpreting the Quantum World. Cambridge: Cambridge University Press, 1997

15. Bunce, L.J., Wright, J.D.M.: The Mackey-Gleason problem for vector measures on projections in von Neumann algebras. J. London Math. Soc. (2) 49(1), 133-149 (1994)

16. Bunce, L.J., Wright, J.D.M.: The quasi-linearity problem for $C^{*}$-algebras. Pac. J. Math. 172(1), 41-47 (1996)

17. Butterfield, J.: Some worlds of quantum theory. In: J. Polkinghorne, R. Russell, eds. Quantum Mechanics (Scientific Perspectives on Divine Action Vol. 5), Rome: Vatican Observatory Publications, 2002, pp. 111-140

18. Butterfield, J.: Topos theory as a framework for partial truth. In: J. Wolenski, P. Gärdenfors, K. KijaniaPlacek, eds. In the Scope of Logic, Methodology and Philosophy of Science, Berlin-HeidelbergNew York: Springer, 2003, pp. 307-329

19. Butterfield, J., Hamilton, J., Isham, C.J.: A topos perspective on the Kochen-Specker theorem: III. Von Neumann algebras as the base category. Int. J. Theor. Phys. 39(6), 1413-1436 (2000)

20. Butterfield, J., Isham, C.J.: A topos perspective on the Kochen-Specker theorem: I. Quantum states as generalized valuations. Int. J. Theor. Phys. 37(11), 2669-2733 (1998)

21. Butterfield, J., Isham, C.J.: A topos perspective on the Kochen-Specker theorem: II. Conceptual aspects and classical analogues. Int. J. Theor. Phys. 38(3), 827-859 (1999)

22. Caspers, M.: Gelfand spectra of $C^{*}$-algebras in topos theory. M.Sc. Thesis Radboud University Nijmegen, available at http://www.math.ru.nl/ landsman/scriptieMartijn.pdf, 2008

23. Caspers, M., Heunen, C., Landsman, N.P., Spitters, B.: Intuitionistic quantum logic of an n-level system. Found. Phys. 39, 731-759 (2009)

24. Cederquist, J., Coquand, T.: Entailment relations and distributive lattices. In: Logic Colloquium '98 (Prague), Volume 13 of Lect. Notes Log., Urbana, IL: Assoc. Symbol. Logic, 2000, pp. 127-139

25. Connes, A.: Noncommutative geometry. San Diego, CA: Academic Press Inc., 1994

26. Connes, A., Marcolli, M.: Noncommutative Geometry, Quantum Fields and Motices, Volume 55 of Colloquium Publications. Providence, RI: Amer. Math. Soc. 2008

27. Coquand, T.: About Stone's notion of spectrum. J. Pure Appl. Alg. 197, 141-158 (2005)

28. Coquand, T., Sambin, G., Smith, J., Valentini, S.: Inductively generated formal topologies. Ann. Pure Appl. Logic 124, 71-106 (2003)

29. Coquand, T., Spitters, B.: Constructive Gelfand duality for $C^{*}$-algebras. Mathematical Proceedings of the Cambridge Philosophical Society, 2009. doi:10.1017/S0305004109002515

30. Coquand, T., Spitters, B.: Integrals and valuations. J. Logic and Anal. 1(3), 1-22 (2009) 
31. Coquand, T., Spitters, B.: Formal topology and constructive mathematics: the Gelfand and Stone-Yosida representation theorems. J. Univ. Comp. Sci. 11(12), 1932-1944 (2005)

32. Döring, A.: Kochen-Specker theorem for Von Neumann algebras. Int. J. Theor. Phys. 44(2), 139-160 (2005)

33. Döring, A.: Quantum states and measures on the spectral presheaf. http://arxiv.org/abs/0809. 4847V1 [quant-ph], 2008, to appear in special issue of Adv. Sci.lett, "Quantum” Parity, Cosmology, Black Holes, M. Bajowld, ed.

34. Döring, A., Isham, C.J.: A topos foundation for theories of physics: I. Formal languages for physics. J. Math. Phys. 49, 053515 (2008)

35. Döring, A., Isham, C.J.: A topos foundation for theories of physics: II. Daseinisation and the liberation of quantum theory. J. Math. Phys. 49, 053516 (2008)

36. Döring, A., Isham, C.J.: A topos foundation for theories of physics: III. The representation of physical quantities with arrows. J. Math. Phys. 49, 053517 (2008)

37. Döring, A., Isham, C.J.: A topos foundation for theories of physics: IV. Categories of Systems. J. Math. Phys. 49, 053518 (2008)

38. Döring, A., Isham, C.J.: 'What is a thing?': Topos theory in the foundations of physics. In: B. Coecke, ed. New Structures in Physics, Lecture Notes in Physics. Springer, 2009, http://arxiv.org/abs/:0803. 0417v1[quant-ph], 2008

39. Emch, G.G.: Mathematical and conceptual foundations of 20th-century physics, Volume $\mathbf{1 0 0}$ of NorthHolland Mathematics Studies. Amsterdam: North-Holland Publishing Co., 1984

40. Fourman, M.P., Grayson, R.J.: Formal spaces. In: The L. E. J. Brouwer Centenary Symposium, Number 110 in Studies in Logic and the Foundations of Mathematics, Amsterdam: North-Holland, 1982, pp. 107-122

41. Fourman, M.P., Ščedrov, A.: The "world's simplest axiom of choice” fails. Manus. Math. 38(3), 325-332 (1982)

42. Goldblatt, R.: Topoi, the Categorical Analysis of Logic. Amsterdam: North-Holland, 1984

43. Grothendieck, A.: Récoltes et Semailles, témoignage sur un passé de mathématicien. Université des Sciences et Techniques du Languedoc, Montpellier, 1985-1987

44. Haag, R.: Local Quantum Physics. Texts and Monographs in Physics. Second, Berlin: Springer-Verlag, 1996

45. Hamhalter, J.: Traces, dispersions of states and hidden variables. Found. Phys. Lett. 17(6), 581-597 (2004)

46. Heckmann, R.: Probabilistic power domains, information systems, and locales. Lect. Notes Comput. Sci. 802, 410-437 (1994)

47. Heunen, C., Landsman, N.P., Spitters, B.: Bohrification of operator algebras and quantum logic. Synthese, to appear; http://arxiv.org/abs/0905.2275

48. Johnstone, P.T.: Stone Spaces. Cambridge: Cambridge University Press, 1982

49. Johnstone, P.T.: Open locales and exponentiation. In: J.W. Gray, ed. Mathematical Applications of Category Theory, Number 30 in Contemporary Mathematics, Providence, RI: Amer. Math. Soc. 1984, pp. 84-116

50. Johnstone, P.T.: Sketches of an Elephant: A Topos Theory Compendium, Volume 1. London: Clarendon Press, 2002

51. Johnstone, P.T.: Sketches of an Elephant: A Topos Theory Compendium, Volume 2. London: Clarendon Press, 2002

52. Joyal, A., Moerdijk, I.: Toposes are cohomologically equivalent to spaces. Amer. J. Math. 112(1), 87-95 (1990)

53. Joyal, A., Tierney, M.: An Extension of the Galois Theory of Grothendieck. Memoirs of the American Mathematical Society, Vol. 309, Providence, RI: Amer. Math. Soc., 1984

54. Kalmbach, G.: Orthomodular Lattices, Volume 18 of London Mathematical Society Monographs. London-New York: Academic Press, 1983

55. Kalmbach, G.: Measures and Hilbert Lattices. Singapore: World Scientific, 1986

56. Kochen, S., Specker, E.: The problem of hidden variables in quantum mechanics. J. Math. Mech. 17, 59-87 (1967)

57. Krömer, R.: Tool and Object: a History and Philosophy of Category Theory. Basel-Boston: Birkhäuser, 2007

58. Landsman, N.P.: Mathematical Topics between Classical and Quantum Mechanics. Berlin-HeidebergNew York: Springer, 1998

59. Landsman, N.P.: Lecture notes on $C^{*}$-algebras and $K$-theory. Available at http://www.science.uva.nl/ $\sim$ npl/CK.pdf, 2004

60. Landsman, N.P.: Between classical and quantum. In: J. Earman J. Butterfield, eds, Handbook of Philosophy of Science, Volume 2: Philosophy of Physics, Oxford: Elsevier, 2007, pp. 417-553

61. Landsman, N.P.: Macroscopic observables and the Born rule. I. Long run frequencies. Rev. Math. Phys. 20, 1173-1190 (2008) 
62. Luxemburg, W.A.J., Zaanen, A.C.: Riesz Spaces. Vol. I. Amsterdam: North-Holland Publishing Co., 1971

63. Mac Lane, S., Moerdijk, I.: Sheaves in Geometry and Logic. Berlin-Heidelberg-New York: Springer, 1992

64. Mackey, G.W.: The Mathematical Foundations of Quantum Mechanics. Mathematical Physics Monograph Series. Advanced Book Program, Reading, MA: Benjamin/Cummings Publishing Co. Inc., 1980

65. McLarty, C.: The uses and abuses of the history of topos theory. British J. Philos. Sci. 41(3), 351-375 (1990)

66. Moerdijk, I.: Spaced spaces. Comp. Math. 53(2), 171-209 (1984)

67. Mulvey, C.J. \& Rend. Circ. Mat. Palermo (2) Suppl., 12, 99-104 (1986). Second topology conference (Taormina, 1984)

68. Mulvey, C.J., Pelletier, J.W.: A geometric characterization concerning compact, convex sets. Math. Proc. Camb. Philos. Soc. 109(2), 351-361 (1991)

69. Negri, S.: Continuous domains as formal spaces. Math. Struct. Comp. Sci. 12(1), 19-52 (2002)

70. Rédei, M.: Quantum Logic in Algebraic Approach, Volume 91. Dordredit: Kluwer Academic Publishers, 1998

71. Sambin, G.: Intuitionistic formal spaces - a first communication. In: D. Skordev, ed. Mathematical Logic and its Applications, London: Plenum, 1987, pp. 187-204

72. Sambin, G.: Some points in formal topology. Theor. Comp. Sci. 305, 347-408 (2003)

73. Scheibe, E.: The Logical Analysis of Quantum Mechanics. Oxford: Pergamon Press, 1973

74. Scott, D.: Lattice theory, data types and semantics. In: NYU Symposium on Formal Semantics (ed. R. Rustin), New York: Prentice-Hall, 1972, pp. 65-106

75. Spitters, B.: Constructive algebraic integration theory without choice. In: T. Coquand, H. Lombardi, and M.-F. Roy, eds. Mathematics, Algorithms, Proofs, Number 05021 in Dagstuhl Seminar Proceedings. IBFI, 2005

76. Varadarajan, V.S.: Geometry of Quantum Theory. Second edition, Berlin-Heidelberg-New York: Springer, 1985

77. Vickers, S.: Topology via Logic. Cambridge: Cambridge University Press, 1989

78. Vickers, S.: The double powerlocale and exponentiation: a case study in geometric logic. Theory Appl. Categ. 12, 372-422 (2004)

79. Vickers, S.: Compactness in locales and in formal topology. Ann. Pure Appl. Logic 137, 413-438 (2006)

80. Vickers, S.: Locales and toposes as spaces. In: M. Aiello, I.E. Pratt-Hartmann, J.F.A.K. van Benthem, eds. Handbook of Spatial Logics, Chapter 8, Berlin-Heidelberg-New York: Springer, 2007

81. Vickers, S.: A localic theory of lower and upper integrals. Mathe. Logic Quart. 54, 109-123 (2008)

82. Zaanen, A.C.: Riesz Spaces. II, Volume 30 of North-Holland Mathematical Library. Amsterdam: North-Holland Publishing Co., 1983

Communicated by Y. Kawahigashi 